\title{
Elasticity Boundary-Value Problems for Straight Wedge Disclinations. A Review on Methods and Results
}

\author{
A.E. Romanov ${ }^{1,2}$ and A.L. Kolesnikova ${ }^{1,3}$ \\ ${ }^{1}$ ITMO University, Kronverkskiy pr, 49, St. Petersburg, 197101, Russia \\ ${ }^{2}$ Ioffe Physical-Technical Institute, Russian Academy of Sciences, Politekhnicheskaya str., 26, St. Petersburg, 194021, Russia \\ ${ }^{3}$ Institute for Problems in Mechanical Engineering, V.O., Bolshoj pr., 61, Russian Academy of Sciences, \\ St. Petersburg, 199178, Russia
}

Received: March 10, 2021

\begin{abstract}
The review presents up-to-date information on the analytical solutions of the isotropic elasticity boundary-value problems for straight wedge disclinations. The considered plane elasticity problems include those for disclinations in uniform or two-phase cylinders, at a free surface of a half-space, and in a plate of finite thickness. Three-dimensional problems under analysis deal with wedge disclinations in a bulk sphere or spherical layer or with the defects with the lines being normal to a free surface of a half-space or to surfaces of the plate. Applications of the given solutions to explanation and prediction of various structure dependent properties of solids are briefly discussed.
\end{abstract}

\section{Introduction}

\section{Background}

2.1. Foundations of linear isotropic elasticity

2.2. Boundary conditions for elasticity problems

2.3. Geometry and elasticity of line/surface defects in an infinite continuum

3. Straight wedge disclinations in infinite elastic media

3.1. Stresses for a straight wedge disclination in an infinite isotropic continuum

3.2. Quadrupoles of wedge disclinations

4. Wedge disclinations in an elastic cylinder

4.1. Wedge disclination in a hollow cylinder

4.2. Singular wedge disclinations in a cylinder

4.3. Wedge disclination in a two-phase cylinder

5. Plane elasticity for disclinations near planar interfaces

5.1. Wedge disclination at a free surface

5.2. Disclinations near interfaces

5.3. Disclinations in the plate of a finite thickness 6. 3D elasticity boundary-value problems for wedge disclinations

6.1. Disclinations normal to the surface of a halfspace
6.2. Wedge disclinations in the plate of a finite thickness

6.3. Wedge disclinations in bodies with spherical geometry

6.3.1. Disclination piercing a spherical layer

6.3.2. Disclination in a bulk sphere

6.3.3. Disclination running through spherical cavity

6.4. Conic disclinations in an elastic sphere

7.Applications

7.1. Disclinations and rotational plasticity

7.2. Disclinations and grain boundaries

7.3. Crack nucleation at disclinations. Diffusion in an elastic field of disclinations

7.4. Disclinations in pentagonal rods and icosahedral particles

7.5. Disclinations and amorphous state

7.6. Domains in ferroelastic films

7.7. Wedge disclinations in graphene

8. Summary and concluding remarks

Acknowledgements

References

Corresponding author: A.E. Romanov, e-mail: alexey.romanov@niuitmo.ru

(C) ITMO University, 2021 


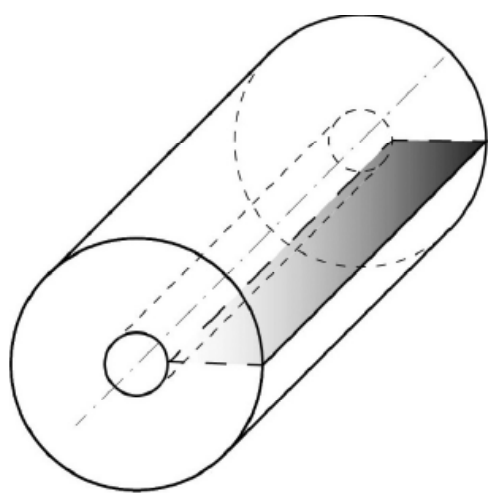

(a)

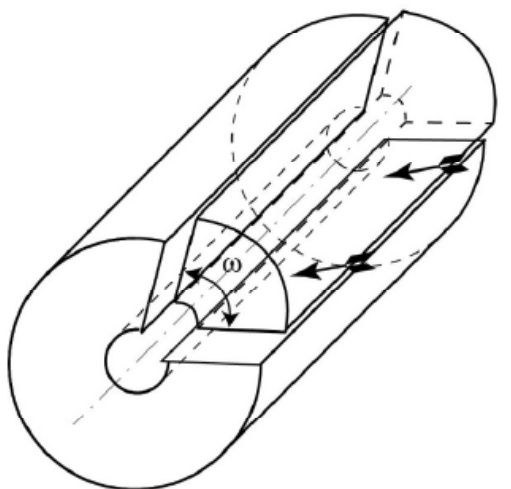

(c)

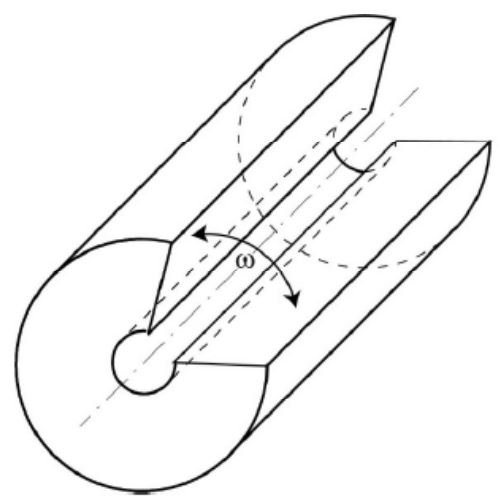

(b)

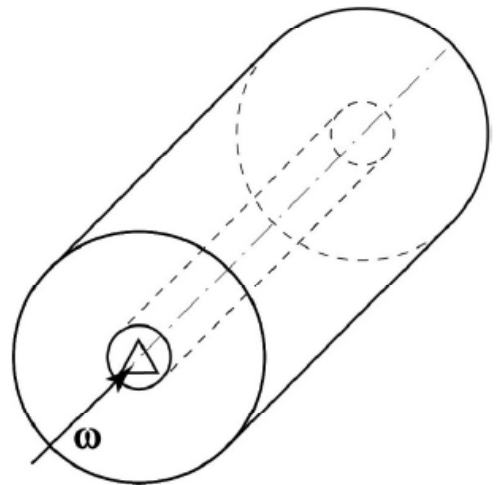

(d)

Fig. 1. Volterra process for the formation of negative wedge disclination in a hollow elastic cylinder. (a) Initial cylinder with a cut; (b) relative rotation of the cut surfaces about cylinder axis with the formation of wedge-like gap; (c) insertion of the sector of the material in the gap; (d) final configuration of disclinated cylinder with internal elastic strains and mechanical stresses.

\section{INTRODUCTION}

Nowadays, disclination approach demonstrates its importance in the description of various phenomena in condensed matter, for valuable reviews we address the reader to Refs. [1-6]. It is good to know that the notation 'disclination' (or originally 'disinclination') were first introduced by Frank in 1958 [7] when considering singular lines and points, i.e. defects, in the orientation field of rod-like molecules of nematic liquid crystals. Similarly, disclinations can be found in the nonuniform distributions of spins in magnetics of different type [2,3].

However, disclinations were explored much earlier in mechanics of deformable solids when Volterra [8] investigated the stress-strain states of unloaded hollow elastic cylinder with multi-value displacement field that corresponds to a solid body motion. Volterra called such states distorsions $(F r$.), which result from the procedure of the cutting of the cylinder in the plane parallel to the cylinder axis, translating or rotating relatively the surfaces of the cut, and then gluing the surface in the final configuration. It is assumed that in the described process (now known as Volterra process) the material is inserted in the emerging empty spaces or taken out from the regions of material overlap. Fig. 1 shows the example of the realization of Volterra process for a wedge disclination. In this review article, we focus on such type of Volterra distorsions only. For a sake of completeness, we have also to mention straight-linear twist disclinations, screw dislocations, and edge dislocations $[1,4]$. Wedge disclination is characterized by the axial vector $\omega-$ Frank vector, which is parallel to the cylinder axis. Its magnitude is disclination strength and is equal to the angle of mutual rotation of the surfaces of the cut in the process of the disclination formation. Depending on the sense of the cut surfaces rotation wedge disclinations can be positive or negative: for the negative ones, the wedge of material is inserted during their formation, whereas for positive ones, the wedge is extracted [4].

As it will be demonstrated below straight-linear wedge disclinations possess remarkable properties: when placed in an imaginary infinite elastic continuum single disclination generates elastic strains and mechanical stresses that diverge both in the defect core, and at 
large distance from a disclination line [4,6]. Such unphysical features can be however corrected with reducing disclination elastic fields either by self-screening in the disclination ensembles of alternating (plus and minus) signs, or by external screening under the influence by free surfaces of the finite size elastic bodies $[4,9,10]$. The second type of screening for wedge disclinations is considered in detail in this review.

In Section 2, we provide a necessary background on the linear elasticity for defects in isotropic continuum and discuss possible types of boundary conditions used for the solution of elasticity boundary-value problems. Then, in Section 3, we give useful formulas for mechanical stresses for a single wedge disclination in an infinite elastically isotropic medium. In Section 4, we start with classical elasticity solutions for a disclination in a hollow isotropic elastic cylinder and later consider a disclinated bulk cylinder that can be also radially nonuniform. In Section 5, we give solutions of plane elasticity problems for disclinations in the case of planar interfaces. Section 6 is devoted to spatial elasticity problems solved for wedge disclinations. Finally, in Section 7 we briefly discuss applications of the listed solutions in various physical models that involved wedge disclinations. We conclude with a brief Summary.

\section{BACKGROUND}

\subsection{Foundations of linear isotropic elasticity}

We are interesting in the solutions of linear isotropic elasticity problems for disclinations. In the linear elasticity approach, displacements components $u_{i}$ are related to the components of symmetric strain tensor $\varepsilon_{k m}$ by:

$$
\varepsilon_{k m}=\frac{1}{2}\left(\frac{\partial u_{k}}{\partial x_{m}}+\frac{\partial u_{m}}{\partial x_{k}}\right),
$$

where Cartesian coordinates $x_{m}$ are used and $i, m, k=1,2,3$.

Hooke's law connects the components $\sigma_{i j}$ of the symmetric mechanical stress tensor with elastic strain components $\varepsilon_{k m}$ :

$$
\begin{aligned}
& \sigma_{i j}=2 G\left(\varepsilon_{i j}+\frac{v}{1-2 v} \varepsilon \delta_{i j}\right) ; \\
& \varepsilon_{i j}=\frac{1}{2 G}\left(\sigma_{i j}-\frac{v}{1+v} \sigma \delta_{i j}\right),
\end{aligned}
$$

where the elastic constants for an isotropic elastic body: shear modus $G$ and Poisson's ratio $v$, are introduced, $\delta_{i j}$ is the Kronecker's delta, $\varepsilon=\varepsilon_{i j}$ is the trace of strain tensor, $\sigma=\sigma_{k k}$ is the trace of stress tensor. Note that in the definition of tensor traces and in the following Einstein's summation rule is applied.
Stresses in the body interior in the absence of volume forces obeys the following equations of equilibrium:

$$
\frac{\partial \sigma_{i j}}{\partial x_{i}}=0 .
$$

All together the system (1) to (3) has 15 equations for 15 quantities. Its analysis is the subject of linear theory of elasticity, when in standard cases compatible strain fields in single-connected elastic bodies are investigated [11]. These cases deal, for examples, with examining strain-stress of the bodies of various geometries loaded by external forces or with given displacements on a part of the body, i.e., surface, line, or point.

Elasticity equations work also for the bodies with internal mechanical stresses caused by eigenstrains (self-strains) of various origin [12]. Typical example of eigenstrain gives thermal expansion; the other important example is related to the spontaneous deformation that appears because of a phase transformation. For us, the most interesting will be consideration of disclinations as carriers of rotational plastic eigenstrain.

\subsection{Boundary conditions for elasticity problems}

It is common in the theory of elasticity to subdivide boundary-value problems into two main classes: when on the surface that bound the considered elastic volume are prescribed as function of coordinates $x_{m}$ either (i) tractions (forces) $\mathbf{T}\left(x_{m}\right)$ or (ii) displacements $\mathbf{u}\left(x_{m}\right)$.

From a view of practical applications, the most important are the following boundary-value problems.

A) Elastic body loaded with external applied forces $\mathbf{T}$. For this case, the conditions

$\left.\sigma_{k l} n_{l}\right|_{S}=T_{k}$

hold with $n_{l}\left(x_{i}\right)$ being the components of the surface $S$ normal unit vector $\mathbf{n}$ at each point $x_{i}$ of the surface. Note that the surface $S$ can be either infinite or finite.

For source of internal stresses important are the boundary-value problems with zero tractions:

$T_{k}=\left.\sigma_{k l} n_{l}\right|_{S}=0$.

This corresponds to the presence of unloaded surfaces for an elastic domain containing for example a disclination. Those can be free surfaces of a half-space, a plate, or an infinite cylinder.

B) With the restrictions imposed on the surface displacements. This can be the rigid condition, when on the surface $S$ 


$$
\left.u_{i}\right|_{S}=0
$$

In most general case the displacements on the surface can be given in the form of some (not necessary constant) functions:

$$
\left.u_{i}\right|_{S}=\varphi_{i}\left(x_{k}\right) \text {. }
$$

When used for external surfaces of an elastic domain both conditions A or B together with the system of Eqs. (1) to (3) of linear isotropic elasticity provide a single-valued solution, which also is non-singular in the domain interior. Expanding the conditions A to internal surfaces gives the other class of solutions, e.g. for a Mode I crack, whereas the application of the condition of Eq. (7) to internal surfaces lead to the definition of Somigliana dislocations [13]; we discuss the last case in some details below in Section 2.3.

C) Phase boundaries or interfaces. In this case we assume the presence of the boundary between two domains with different elastic moduli, for example $G_{1}, v_{1}$ and $G_{2}, v_{2}$, with the continuity of displacements and tractions across the interface $S$ :

$$
\begin{aligned}
& \left.u_{i}^{(1)}\right|_{S}=\left.u_{i}^{(2)}\right|_{S} ; \\
& \left.\sigma_{k l}^{(1)} n_{l}\right|_{S}=\left.\sigma_{k l}^{(2)} n_{l}\right|_{S} .
\end{aligned}
$$

The components of stresses that do not appear in Eq. (8b) can be discontinuous at the interface $S$ together with discontinuity of some strain components. In the limit cases, the solutions found with the conditions of Eqs. (8) turn in the solutions for boundary-value problems for a free surface (with $G_{2}=0$ ) or a rigid boundary (with $G_{2}=\infty$ ).

D) Slipping interfaces. Such boundaries do not transmit shear loads, normal stresses and displacements remain continuous across the interface, but tangential displacements can demonstrate discontinuities on surface $S$ :

$$
\begin{aligned}
& \left.u_{i}^{(1)} n_{i}\right|_{S}=\left.u_{i}^{(2)} n_{i}\right|_{S} ; \\
& \left.n_{k} \sigma_{k l}^{(1)} n_{l}\right|_{S}=\left.n_{k} \sigma_{k l}^{(2)} n_{l}\right|_{S} ; \\
& \left.s_{k} \sigma_{k l}^{(1)} n_{l}\right|_{S}=\left.t_{k} \sigma_{k l}^{(1)} n_{l}\right|_{S}=\left.s_{k} \sigma_{k l}^{(2)} n_{l}\right|_{S}= \\
& \left.t_{k} \sigma_{k l}^{(2)} n_{l}\right|_{S}=0,
\end{aligned}
$$

where $s_{k}$ and $t_{k}$ are components of two mutually orthogonal unit vectors $\mathbf{s}$ and $\mathbf{t}$ laying in the plane tangential to the surface $S$.

The surfaces with conditions of Eqs. (9) model the grain boundaries in polycrystals under the realization of grain boundary sliding and cracks of Mode II and Mode III under external loading.

Real physical situations can lead to the combination of boundary conditions on the external surfaces and/or interfaces. The obvious examples are a crack at the phase boundary or a disclination in a thin layer deposited on the substrate with different elastic properties. It is useful to note that, in general, boundary-value problems can be classified as well-posed and ill-posed ones [14]. The well-posed problem can be solved (it least theoretically) and give a unique solution, whereas ill-posed problems usually do not have enough mathematical conditions to find the solution. In this last case additional physical arguments can be applied to turn the ill-posed boundary value problem to practical results.

\subsection{Geometry and elasticity of line/surface defects in an infinite continuum}

Setting the conditions for displacements on internal surface one can define a defect of general line/surface type-Somigliana dislocation [13], as the generalization of the Volterra process given in Fig. 1. For Somigliana loop dislocation shown schematically in Fig. 2, we first select the spatial configuration of the cut surface $\Gamma$ resting on the defect line $L$. Then the surfaces of the cut experience relative displacement $\left.\mathbf{u}(\mathbf{r})\right|_{\Gamma}$, which is the function on the position $\mathbf{r}$ on $\Gamma$. As it was already noted for Volterra process in Introduction, the material is inserted in empty spaces (voids) or taken out from the regions of material overlap; this is shown schematically in Fig. 2b.

Volterra distorsions, namely translation dislocations with their main characteristic known as Burgers vector b and disclinations characterized by Frank (rotation) vector $\omega$ and the position in space $\mathbf{r}^{\omega}$ of the axis of this rotation vector can be viewed as special case of Somigliana dislocations with

$$
\left.\mathbf{u}\right|_{\Gamma}=-\mathbf{b}+\boldsymbol{\omega} \times\left(\mathbf{r}^{\omega}-\mathbf{r}\right)
$$

or in component form

$\left.u_{i}\right|_{\Gamma}=-b_{i}+\epsilon_{i j k} \omega_{j}\left(x_{k}^{\omega}-x_{k}\right)$,

where $\epsilon_{i j k}$ is the permutation symbol, $x_{k}$ are components of the radius-vector $\mathbf{r}$, and $x_{k}^{\omega}$ are the components of the vector that ends at a point of the line defining the axis of rotation. It is important to note that the stress-strain state for elastic solids with Volterra distorsions does not depend on the position in space of the surface $\Gamma$ that is used in the defect definition $[1,4]$ and therefore dislocations and disclinations are considered as line defects. For a general Somigliana dislocation the choice of the surface $\Gamma$ influences the distribution and continuity of some components of stresses and strains. 


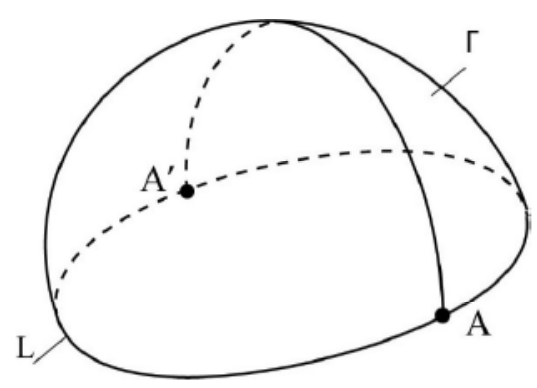

(a)

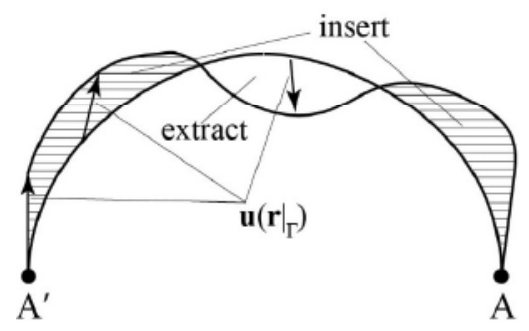

(b)

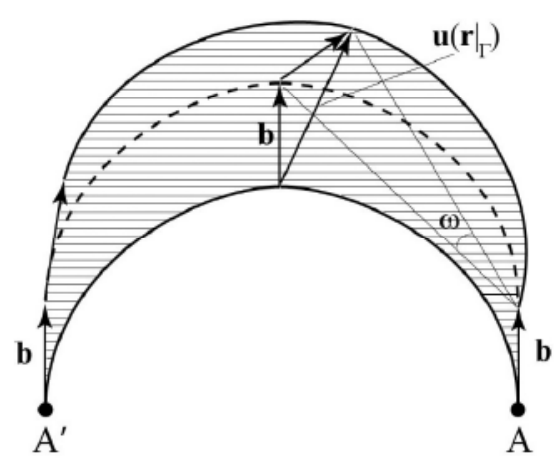

(c)

Fig. 2. Line/surface defect with discontinuity of displacement $\left.\mathbf{u}(\mathbf{r})\right|_{\Gamma}$. (a) Surface $\mathrm{G}$ resting on a closed defect line $L$; displacements of the cut surfaces for Somigliana (b) and Volterra (c) dislocations; in dashed region the material is inserted.

Using different "language", dislocations and disclinations can be introduced in continuum with the help of self-distortion $\beta_{i j}^{*}$ [12]. For example, in the case of the planar disclination loop laying in the plane $x_{p}$ its self-distortion can be written as [15]:

$\beta_{p i}^{*}=\epsilon_{i j k} \omega_{j}\left(x_{k}^{\omega}-x_{k}\right) \delta\left(x_{p}\right) H(\Gamma)$,

where $\delta(z)$ is the Dirac delta-function and $H(\Gamma)$ is the Heaviside step-function defined for the part of the plane bounded by the line of the loop.

In an elastic material, an isolated disclination can be also defined as the line defect generating constant vector for the integral [16]

$\omega_{m}=\oint_{\gamma} l_{n} \kappa_{m n}$,

where $\kappa_{m n}$ are the components of elastic bend-twist tensor [17] and the integration is performed along an arbitrary contour $\gamma$ that captures the defect line.

It was shown by Mura $[12,18,19]$ that in linear elastic continuum the elastic fields of isolated disclinations and dislocations can be found on the base of the total displacements $u_{m}^{T}$ given by the following formula:

$u_{m}^{T}\left(\mathbf{r}^{\prime}\right)=\int_{V} \beta_{i j}^{*}(\mathbf{r}) C_{j i k l} \frac{\partial G_{k m}\left(\mathbf{r}^{\prime}-\mathbf{r}\right)}{\partial x_{l}^{\prime}} \mathrm{d} V$ where $C_{i j k l}$ are the elastic constants; $G_{k m}\left(\mathbf{r}^{\prime}-\mathbf{r}\right)$ is Green function of the elastic media [12].

Equation (13) can be transformed to the easy-to-use expression [19]:

$$
\begin{aligned}
& u_{m}^{T}\left(\mathbf{r}^{\prime}\right)= \\
& -i \iint_{-\infty}^{\infty} \int \xi_{i} \hat{\beta}_{i j}^{*} C_{j k l k} L_{k m} \exp \left(i \xi \cdot \mathbf{r}^{\prime}\right) \mathrm{d} \xi_{x} \mathrm{~d} \xi_{y} \mathrm{~d} \xi_{z} .
\end{aligned}
$$

Here $\hat{\beta}_{i j}^{*}$ and $L_{k m}$ are Fourier transforms of the tensors $\beta_{i j}^{*}$ and $G_{k m}$, correspondingly:

$$
\begin{aligned}
& \hat{\beta}_{i j}^{*}=(\sqrt{2 \pi})^{-3} \iint_{-\infty}^{\infty} \int \beta_{i j}^{*} \exp \left(-i \xi \cdot \mathbf{r}^{\prime}\right) \mathrm{d} x^{\prime} \mathrm{d} y^{\prime} \mathrm{d} z^{\prime} ; \\
& L_{m k}=(\sqrt{2 \pi})^{-3} \iint_{-\infty}^{\infty} \int G_{m k} \exp \left(-i \xi \cdot \mathbf{r}^{\prime}\right) \mathrm{d} x^{\prime} \mathrm{d} y^{\prime} \mathrm{d} z^{\prime} .
\end{aligned}
$$

For isotropic continuum

$$
\begin{aligned}
& C_{j i k l}=G\left[2 v \delta_{j i} \delta_{k l} /(1-2 v)+\delta_{i k} \delta_{j l}+\delta_{i l} \delta_{j k}\right], \\
& L_{m k}=(\sqrt{2 \pi})^{-3}\left[2(1-v) \xi^{2} \delta_{m k}-\xi_{m} \xi_{k}\right] /\left[2 G(1-v) \xi^{4}\right],
\end{aligned}
$$

$\delta_{i j}$ is the Kronecker's symbol, $G$ is a shear modulus, $v$ is the Poisson's ratio, $\xi^{2}=\xi_{x}^{2}+\xi_{y}^{2}+\xi_{z}^{2}$. 


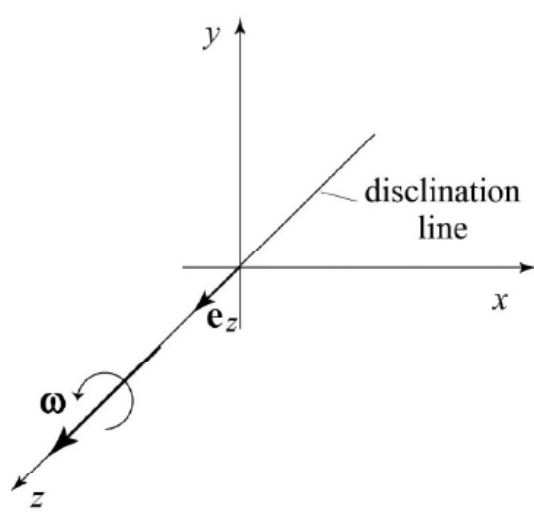

(a)

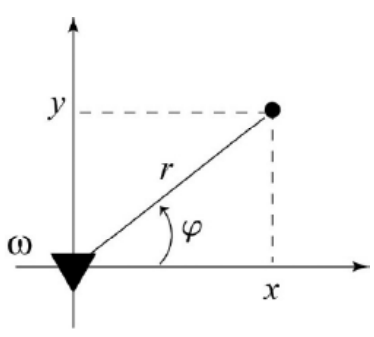

(b)

Fig. 3. Geometry and coordinate systems used in calculation of elastic fields of wedge disclinations in isotropic elastic media. (a) a straight wedge disclination with Frank vector $\omega=\omega \varepsilon_{z}$; (b) cylindrical $r, \varphi, z$ and Cartesian $x, y, z$ coordinate systems associated with disclination line.

Total displacements provide a possible way to find total distortions $\beta_{m p}^{T}$ and total strains $\varepsilon_{m p}^{T}$, then elastic strains $\varepsilon_{m p}$ and stresses via Hook law (for isotropic media defined via Eq. (2a)):

$\beta_{m p}^{T}=\frac{\partial u_{m}^{T}}{\partial x_{p}}=$

$\iiint \xi_{l} \xi_{p} \hat{\beta}_{i j}^{*} C_{j i k l} L_{k m} \exp \left(i \xi \cdot \mathbf{r}^{\prime}\right) \mathrm{d} \xi_{x} \mathrm{~d} \xi_{y} \mathrm{~d} \xi_{z}$,

$\varepsilon_{m p}^{T}=\frac{1}{2}\left(\beta_{m p}^{T}+\beta_{p m}^{T}\right)$,

$\varepsilon_{m p}=\varepsilon_{m p}^{T}-\varepsilon_{m p}^{*}$,

where $\varepsilon_{m p}^{*}=\left(\beta_{m p}^{*}+\beta_{p m}^{*}\right) / 2$.

It is known that for straight-linear defects $[9,20]$ and loops with rectangular geometry $[9,21]$ elastic fields can be expressed in a closed form including only elementary functions, whereas for circular loop defects special functions will be involved $[9,15,22]$.

\section{STRAIGHT WEDGE DISCLINATIONS IN INFINITE ELASTIC MEDIA}

\subsection{Stresses for a straight wedge disclination in an infinite isotropic continuum}

Consider a straight disclination whose line goes along the axis $z$ of Cartesian coordinate system $x, y, z$ (we also use related cylindrical coordinates $r, \varphi, z)$. For a wedge disclination with $\mathbf{r}^{\omega}=0$ Frank vector coincides with the $z$-axis, too, as it is shown in Fig. 3. It is obvious that for $r \rightarrow \infty$, displacements given by Eq. (10) acquire infinite values. In turn, this leads to infinite (unphysical) distortions in an elastic continuum resulting from the realization of Volterra process. Self-distortion of such wedge disclination can be written in the following form:

$\beta_{y y}^{*}=-\omega x \delta(y) H(x)$

Here we have chosen a half-plane $(x>0, y=0)$ as the cut surface $\Gamma, \omega=\omega \boldsymbol{e}_{z}$ with $\omega$ being the disclination strength.

Exploring Eqs. (15), (16), and (2a) one finds the stresses for a wedge disclination in an elastic infinite medium. In Cartesian coordinates the stresses for a straight wedge disclination are [20]:

$\sigma_{x x}=\frac{G \omega}{2 \pi(1-v)} \times$
$\left(\frac{1}{2} \log \left(x^{2}+y^{2}\right)+\frac{y^{2}}{x^{2}+y^{2}}+\frac{v}{1-2 v}\right)$,

$\sigma_{y y}=\frac{G \omega}{2 \pi(1-v)} \times$

$\left(\frac{1}{2} \log \left(x^{2}+y^{2}\right)+\frac{y^{2}}{x^{2}+y^{2}}+\frac{v}{1-2 v}\right)$,

$\sigma_{z z}=v\left(\sigma_{x x}^{\infty}+\sigma_{y y}^{\infty}\right)=$

$\frac{v G \omega}{2 \pi(1-v)}\left(\log \left(x^{2}+y^{2}\right)+\frac{1}{1-2 v}\right)$,

$\sigma_{x y}=-\frac{G \omega}{2 \pi(1-v)} \frac{x y}{x^{2}+y^{2}}$,

where $\omega$ is the disclination strength (or charge) - the magnitude of Frank vector with the sign "+" or "-" de- 
pending on the manipulation with the material during Volterra process with "-" corresponding to the inserted wedge of material. Zero components of stresses are omitted in Eqs. (17) (and throughout the whole text); the form of the component $\sigma_{z z}$ is caused by the state of plane strain for a straight wedge disclination.

Because of rotational nature of disclination defects the stresses have the simplest representation (no dependence on polar angle $\varphi$ ) in cylindrical coordinates:

$$
\begin{aligned}
& \sigma_{r r}=\frac{G \omega}{2 \pi(1-v)}\left(\log r+\frac{v}{1-2 v}\right) \\
& \sigma_{\varphi \varphi}=\frac{G \omega}{2 \pi(1-v)}\left(\log r+\frac{1-v}{1-2 v}\right) \\
& \sigma_{z z}=v\left(\sigma_{r r}+\sigma_{\varphi \varphi}\right)=\frac{v G \omega}{2 \pi(1-v)}\left(2 \log r+\frac{1}{1-2 v}\right)
\end{aligned}
$$

where $r^{2}=x^{2}+y^{2}$.

Found stresses satisfy equilibrium Eqs. (3) and demonstrate typical features that are peculiar to disclinations only. Elastic strains and displacements leading to the relations for stresses defined by Eqs. (17) or (18) obey conditions of Eqs. (10) and (12). Normal stresses of single wedge disclination diverge as $\log r$ both for $r \rightarrow \infty$ and $r \rightarrow 0$. The logarithmic dependence of stresses on coordinates is unphysical because log-function can operate only dimensionless variables but not the coordinates with the dimension of length. This means that the coordinates in Eqs. (17) and (18) should be normalized to some length. Such a normalization for straight disclinations is possible in the case of multipole disclination systems or for disclinations in the bodies of finite size. The analysis of the second possibility is the main subject of this review. In general, the relations for elastic fields of disclinations in infinite continuum can be only used as building blocks in construction of the relations for multipole configurations and as starting point for finding the solutions of elasticity boundary-value problems.

It is well known that in the case of plane strain, i.e., $\varepsilon_{z z}=0$, three independent components of stresses can be found with the help of Airy stress function $\chi$, which satisfies the biharmonic differential equation [11]:

$$
\Delta \Delta \chi=0
$$

$\sigma_{x x}=\frac{\partial^{2} \chi}{\partial y^{2}}, \quad \sigma_{y y}=\frac{\partial^{2} \chi}{\partial x^{2}}, \sigma_{x y}=-\frac{\partial^{2} \chi}{\partial x \partial y}$, $\sigma_{z z}=v\left(\sigma_{x x}+\sigma_{y y}\right)$
The case of wedge disclinations fits the condition of plane strain, thus Airy stress function can be advanced to reproduce the stresses from Eqs. (17) by exploring the relationships (20). Such stress function was proposed in Ref. [23]:

$$
\begin{aligned}
\chi= & \frac{G \omega}{4 \pi(1-v)} r^{2} \log r= \\
& \frac{G \omega}{8 \pi(1-v)}\left(x^{2}+y^{2}\right) \log \left(x^{2}+y^{2}\right) .
\end{aligned}
$$

It easy to check that the stresses found with the help $\chi$ coincide with those given by Eqs. (17) with accuracy to constant term. In many cases the solution of elasticity problems for wedge disclinations can be searched having stress function $\chi$ as starting point. It worth to note that stress function of an edge dislocation can be derived from Eq. (17) by differentiation with respect the coordinate $(x$ or $y$ ). The property of representation of an edge dislocation in the form of wedge disclination dipole is useful for the solution of elasticity problems both for dislocations and for disclinations; this feature was explored for the first time in Ref. [23].

\subsection{Quadrupoles of wedge disclinations}

The energy stored in any elastic field can be calculated by integrating elastic energy density over the whole volume of the system:

$E=\frac{1}{2} \int_{V} \sigma_{i j}(\mathbf{r}) \varepsilon_{i j}(\mathbf{r}) \mathrm{d} V$

or in the case of the defect by the equivalent approach developed by Mura [12] basing on the consideration of plastic distortion $\beta_{i j}^{*}$ (or eigenstrain $\varepsilon_{i j}^{*}$ ) of the defect:

$$
E=-\frac{1}{2} \int_{V} \beta_{i j}^{*}(\mathbf{r}) \sigma_{i j}(\mathbf{r}) \mathrm{d} V=-\frac{1}{2} \int_{V} \varepsilon_{i j}^{*}(\mathbf{r}) \sigma_{i j}(\mathbf{r}) \mathrm{d} V .
$$

In the case of a single wedge disclination or even in the case of a wedge disclination dipole, which is formed of two opposite sign disclinations, the calculations with Eqs. (22) give infinite value for the elastic energy. However, wedge disclinations quadrupoles provide the example of disclination systems in infinite media with finite stored elastic energy. Such systems, also known as self-screened disclination configurations, are useful for practical applications; they were studied in necessary details in Refs. [4,10,24].

The self-energies for quadrupoles, shown in Fig. 4, have the following algebraic representations:

(a) for the parallelogram (Fig. 4a) 


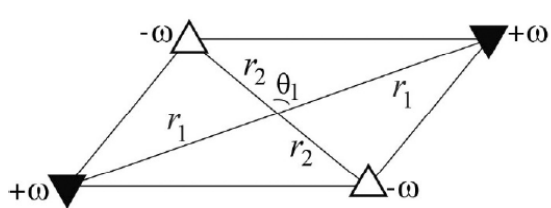

(a)

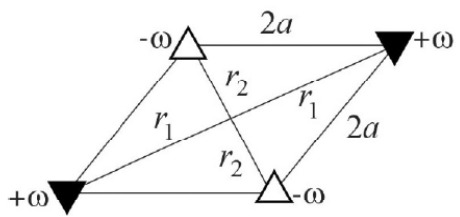

(c)

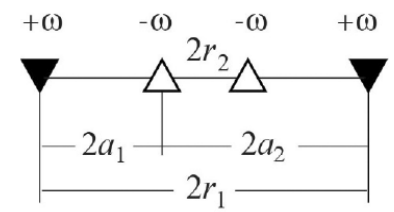

(e)

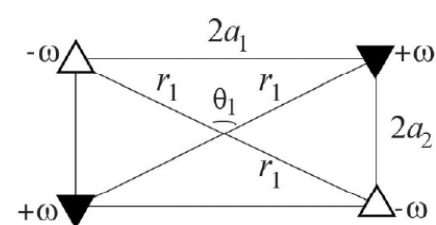

(b)

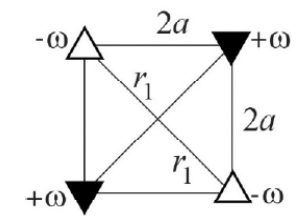

(d)

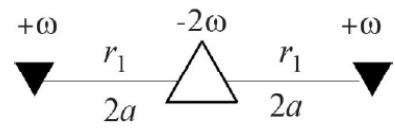

(f)

Fig. 4. Self-screened disclination quadrupoles, adapted from [24]. Parallelogram (a), special cases of parallelogram: a rectangle (b), a rhombus (c), a quadrate (d), and line quadrupoles (e) and (f) as degenerate parallelograms.

$$
\begin{aligned}
& E_{p a r}=\frac{G \omega^{2}}{4 \pi(1-v)}\left(r_{1}^{2} \ln \frac{16 r_{1}^{4}}{\left(r_{1}^{2}+r_{2}^{2}-2 r_{1} r_{2} \cos \theta_{1}\right)\left(r_{1}^{2}+r_{2}^{2}+2 r_{1} r_{2} \cos \theta_{1}\right)}+\right. \\
& \left.r_{2}^{2} \ln \frac{16 r_{2}^{4}}{\left(r_{1}^{2}+r_{2}^{2}-2 r_{1} r_{2} \cos \theta_{1}\right)\left(r_{1}^{2}+r_{2}^{2}+2 r_{1} r_{2} \cos \theta_{1}\right)}+2 r_{1} r_{2} \cos \theta_{1} \ln \frac{r_{1}^{2}+r_{2}^{2}-2 r_{1} r_{2} \cos \theta_{1}}{r_{1}^{2}+r_{2}^{2}+2 r_{1} r_{2} \cos \theta_{1}}\right),
\end{aligned}
$$

(b) for the rectangle (Fig. 4b)

$$
\begin{aligned}
& E_{\text {rec }}=\frac{G \omega^{2} r_{1}^{2}}{2 \pi(1-v)}\left(\ln 4-\left(1-\cos \theta_{1}\right) \ln \left(1-\cos \theta_{1}\right)-\left(1+\cos \theta_{1}\right) \ln \left(1+\cos \theta_{1}\right)\right)= \\
& \frac{G \omega^{2}}{\pi(1-v)}\left(a_{1}^{2} \ln \frac{a_{1}^{2}+a_{2}^{2}}{a_{1}^{2}}+a_{2}^{2} \ln \frac{a_{1}^{2}+a_{2}^{2}}{a_{2}^{2}}\right),
\end{aligned}
$$

(c) for the rhombus (Fig. 4c)

$$
E_{\text {rhomb }}=\frac{G \omega^{2}}{2 \pi(1-v)}\left(r_{1}^{2} \ln \frac{4 r_{1}^{2}}{\left(r_{1}^{2}+r_{2}^{2}\right)}+r_{2}^{2} \ln \frac{4 r_{2}^{2}}{\left(r_{1}^{2}+r_{2}^{2}\right)}\right),
$$

(d) for the square (Fig. 4d)

$$
E_{q u d r}=\frac{G \omega^{2} r_{1}^{2}}{\pi(1-v)} \ln 2=\frac{2 G(1+v) \omega^{2} a^{2}}{\pi} \ln 2,
$$

(e) for the line quadrupole (Fig. 4e)

$$
\begin{aligned}
E_{l q} & =\frac{G \omega^{2}}{2 \pi(1-v)}\left(r_{1}^{2} \ln \frac{4 r_{1}^{2}}{\left(r_{1}^{2}-r_{2}^{2}\right)}+r_{2}^{2} \ln \frac{4 r_{2}^{2}}{\left(r_{1}^{2}-r_{2}^{2}\right)}-2 r_{1} r_{2} \ln \frac{\left(r_{1}+r_{2}\right)}{\left(r_{1}-r_{2}\right)}\right)= \\
& \frac{G \omega^{2}}{\pi(1-v)}\left(a_{1}^{2} \ln \frac{a_{2}^{2}-a_{1}^{2}}{a_{1}^{2}}+a_{2}^{2} \ln \frac{a_{2}^{2}-a_{1}^{2}}{a_{2}^{2}}+2 a_{1} a_{2} \ln \frac{a_{2}+a_{1}}{a_{2}-a_{1}}\right), r_{1}>r_{2}, a_{2}>a_{1},
\end{aligned}
$$




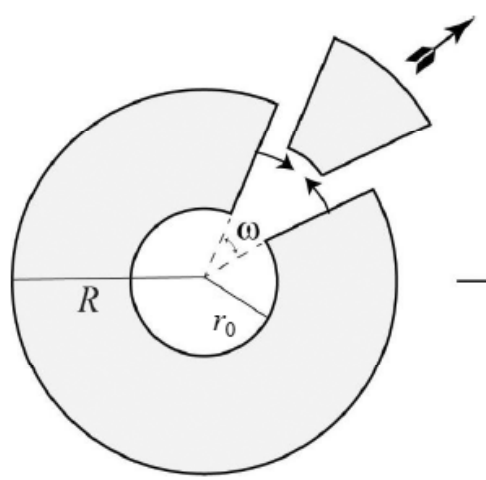

(a)

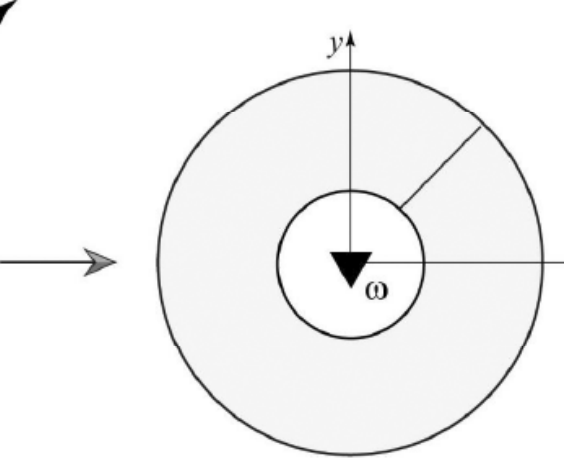

(b)

Fig. 5. Introduction of a positive wedge disclination of strength $w$ in a hollow cylinder. Schematics for cylinder crosssection in the process (a) and after (b) wedge-like material sector extraction.

(f) for the line quadrupole (Fig. 4f)

$$
E_{l q}=\frac{G \omega^{2} r_{1}^{2}}{\pi(1-v)} \ln 2=\frac{4 G \omega^{2} a^{2}}{\pi(1-v)} \ln 2 .
$$

In Eqs. (23), the energies are given per unit length of disclination lines, which spread normally to the plane of schematics in Fig. 4. For the used quadrupole parameters, i.e. $r_{1}, r_{2}, a, a_{1}, a_{2}$, and $\theta_{1}$ we have adopted their meanings in accordance with those shown Fig. 4. Note that in the original work, see Ref. [24], the formulas were given for the case of plane stress, but not plane strain. They are related by a simple renormalization procedure; one just needs to substitute the multiplier $G /(1-v)$ with $G(1+v)$ in Eqs. (23).

\section{WEDGE DISCLINATIONS IN AN ELASTIC CYLINDER}

\subsection{Wedge disclination in a hollow cylinder}

Historically first solved boundary-value problem for disclinations was the problem for a wedge disclination in a hollow elastically isotropic cylinder [8]. It is supposed that a positive wedge disclination is formed when a wedge-like sector of material is taken out from the hollow cylinder with internal and external radii $r_{0}$ and $R$ respectively, as it is schematically shown in Fig. 5. We assume that the edge of the wedge-like sector of angle $\omega$ coincides with cylinder axis. For comparison, for a negative wedge disclination shown in Fig. 1 the wedgelike sector is inserted in the cut cylinder.

Presence of internal and external free lateral cylinder surfaces means that for both of them the boundary conditions for stresses of the type of Eq. (5) hold:

$$
\left.\sigma_{k r}\right|_{\substack{r=r_{0} \\ r=R}}=0, k=r, \varphi, z
$$

The described elasticity boundary-value problem is classical in the theory of elasticity; its solution can be found in numerous elasticity monographs and handbooks. For example, following Ref. [11] we get for stresses:

$$
\begin{aligned}
& \sigma_{r r}=\frac{G \omega}{2 \pi(1-v)}\left(\log \frac{r}{R}-\frac{r_{0}^{2}\left(R^{2}-r^{2}\right)}{r^{2}\left(R^{2}-r_{0}^{2}\right)} \log \frac{r_{0}}{R}\right) ; \\
& \sigma_{\varphi \varphi}=\frac{G \omega}{2 \pi(1-v)}\left(1+\log \frac{r}{R}+\frac{r_{0}^{2}\left(R^{2}+r^{2}\right)}{r^{2}\left(R^{2}-r_{0}^{2}\right)} \log \frac{r_{0}}{R}\right) ;
\end{aligned}
$$

$$
\begin{aligned}
& \sigma_{z z}=v\left(\sigma_{r r}+\sigma_{\varphi \varphi}\right)=\frac{v G \omega}{2 \pi(1-v)} \times \\
& \left(1+2 \log \frac{r}{R}+\frac{2 r_{0}^{2}}{R^{2}-r_{0}^{2}} \log \frac{r_{0}}{R}\right) .
\end{aligned}
$$

Remarkable feature of the disclination solution given by Eqs. (25) is not only complete fulfillment of the boundary conditions

$$
\left.\sigma_{r r}\right|_{\substack{r=r_{0} \\ r=R}} \equiv 0
$$

but also, the absence of the net force in the $z$-direction:

$$
F_{z}=\int_{S} \sigma_{z z}(r) \mathrm{d} S=2 \pi \int_{r_{0}}^{R} \sigma_{z z}(r) r \mathrm{~d} r \equiv 0 .
$$

This means that the found stresses can be used for the analysis of wedge disclination behavior in finite height cylinders with high height/diameter aspect ratios without introduction of additional terms accounting for axial 
loading. Of course, near cylinder ends the solution should be modified to include three-dimentional character stress-strain state that will lead to the appearance of the non-uniform (in dependence on coordinate $r$ ) displacements of cylinder ends. Such an effect is clearly seen in the photographs of wedge disclinations models made from gelatin in seminal Volterra's work, see Ref. [8].

Calculations with the help of Eqs. (22a) or (22b) result in the energy of wedge disclination in the hollow cylinder [25]:

$$
E^{c}=\frac{G \omega^{2} R^{2}}{16 \pi(1-v)}\left(\frac{R^{2}-r_{0}^{2}}{R^{2}}-\frac{4 r_{0}^{2}}{R^{2}-r_{0}^{2}}\left(\log \frac{r_{0}}{R}\right)^{2}\right) \text {. }
$$

Here and below for plane strain problems we write the energies per unit length of a disclination line.

It is obvious that the disclination energy does not depend on its sign. This is a direct consequence of the linearity of elasticity Eqs. (1) to (3) we use. In the considered geometry the disclination energy is controlled by two screening parameters $r_{0}$ and $R$. One limiting case concerns the formation of a disclination in a thin cylindrical shell of constant thickness $\Delta$ with the radius of the shell $R \rightarrow \infty$; under such conditions Eq. (28) acquires the form:

$$
E^{c}=\frac{G \omega^{2} \Delta^{3}}{8 \pi(1-v) R} .
$$

The other limiting case that deals with so-called singular disclinations is considered in detail in the next Section.

\subsection{Singular wedge disclinations in a cylinder}

Relation for stresses of a singular wedge disclination, which line coincides with the axis of the bulk cylinder with external radius $R$, can be determined from Eqs. (25) allowing $r_{0} \rightarrow 0$ and transforming to Cartesian coordinates:

$$
\begin{aligned}
& \sigma_{x x}=\frac{G \omega}{2 \pi(1-v)}\left(\log \frac{r}{R}+\frac{y^{2}}{r^{2}}\right) ; \\
& \sigma_{y y}=\frac{G \omega}{2 \pi(1-v)}\left(\log \frac{r}{R}+\frac{x^{2}}{r^{2}}\right) ; \\
& \sigma_{z z}=v\left(\sigma_{x x}+\sigma_{y y}\right)=\frac{v G \omega}{2 \pi(1-v)}\left(2 \log \frac{r}{R}+1\right) ; \\
& \sigma_{x y}=-\frac{G \omega}{2 \pi(1-v)} \frac{x y}{r^{2}},
\end{aligned}
$$

where $r^{2}=x^{2}+y^{2}$, as before.

There is an obvious similarity with the stresses of singular disclinations in infinite isotropic continuum, see Eqs. (17) and (18): the same logarithmic dependence on $r$ is present with singularity for $r \rightarrow 0$. However, the log-functions in Eqs. (30) are physically correct because radial coordinate is normalized by screening length $R$. Note that stresses for a singular wedge disclination in a cylinder can be obtained with help of the following Airy stress function:

$$
\chi_{0}^{c}=\frac{G \omega}{4 \pi(1-v)}\left(r^{2} \log \frac{r}{R}-\frac{r^{2}}{2}\right) .
$$

The energy of a singular wedge disclination in a cylinder is derived from Eq. (28) in the limit $r \rightarrow 0$ :

$$
E_{0}^{c}=\frac{G \omega^{2} R^{2}}{16 \pi(1-v)} .
$$

It follows that the elastic energy of a singular wedge disclination has no dependence on the core radius (which is equivalent to $r_{0}$ ), to the contrary to well-known result for dislocations [1]. This is related to the linear dependence of displacements of the cut surface in the Volterra process of disclination formation on distance $r$.

For complete analysis of wedge disclination behavior in a cylinder one needs to know the properties of a wedge disclination shifted with respect the axis of a cylinder, see Fig. 6. The solution for stresses of such a disclination, e.g., disclination $\omega_{1}$ displaced from coordinate origin by $r_{1}$ along $x$-axis, is set by the following Airy stress function [26]:

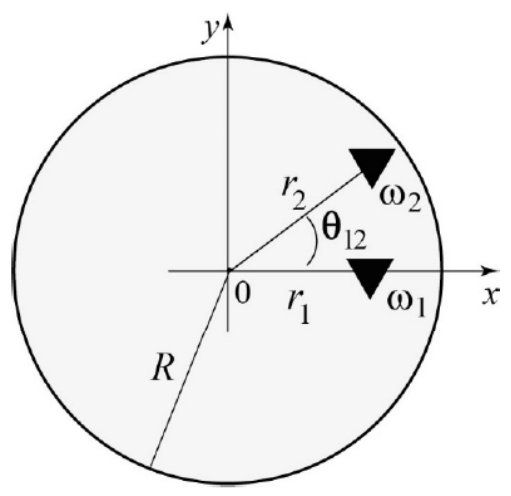

Fig. 6. Schematics for the interaction of wedge disclinations in a cylinder. Two wedge disclinations with strengths $\omega_{1}$ and $\omega_{2}$ are shifted with respect cylinder axis by $r_{1}$ and $r_{2}$, correspondingly; angle $\theta_{12}$ gives relative angular position between disclinations. 


$$
\chi_{r 1}^{c}=\frac{G \omega_{1}}{8 \pi(1-v)}\left[\left(\left(x-r_{1}\right)^{2}+y^{2}\right) \log \frac{R^{2}\left(\left(x-r_{1}\right)^{2}+y^{2}\right)}{\left(x r_{1}-R^{2}\right)^{2}+y^{2} r_{1}^{2}}-\frac{r^{2}\left(r_{1}^{2}-R^{2}\right)}{R^{2}}\right] .
$$

We do not provide here cumbersome formulas for stress tensor components that are derived by exploring Eqs. (33) and (20) but rather discuss the pair interaction energy $E_{12}^{c}$ between two arbitrary placed disclinations in a cylinder, as shown in Fig. 6. This energy can be found as work done during the formation of disclination $\omega_{2}$ in the stress field $\sigma_{i j}^{1}$ of the disclination $\omega_{1}$ :

$$
E_{12}^{c}=\left.\omega_{2} \int_{r_{2}}^{R} \sigma_{\varphi \varphi}^{1}(r, \varphi)\right|_{\varphi=\theta_{12}}\left(r-r_{2}\right) \mathrm{d} r
$$

that gives [26]

$$
E_{12}^{c}=\frac{G \omega_{1} \omega_{2}}{8 \pi(1-v)}\left[\left(r_{1}^{2}+r_{2}^{2}-2 r_{1} r_{2} \cos \theta_{12}\right) \ln \frac{R^{2}\left(r_{1}^{2}+r_{2}^{2}-2 r_{1} r_{2} \cos \theta_{12}\right)}{r_{1}^{2} r_{2}^{2}-2 R^{2} r_{1} r_{2} \cos \theta_{12}+R^{4}}+R^{2}-r_{1}^{2}-r_{2}^{2}+\frac{r_{1}^{2} r_{2}^{2}}{R^{2}}\right] .
$$

From Eq. (35) one can get:

(i) with $r_{2}=r_{1}, \omega_{2}=\omega_{1}$, and $\theta_{12}=0$ the self-energy $E_{\gamma 1}^{c}$ of wedge disclination shifted with respect the cylinder axis:

$$
E_{r 1}^{c}=\frac{1}{2} E_{11}^{c}=\frac{G \omega_{1}^{2}}{16 \pi(1-v)} \frac{\left(R^{2}-r_{1}^{2}\right)^{2}}{R^{2}}
$$

which reproduces for $r_{1}=0$ the energy of unshifted disclination of Eq. (32);

(ii) with $r_{1}=0$ or $r_{2}=0$ the energy of pair interaction of two disclinations when one of them is in the central position in the cylinder; this particular result was found in Ref. [27].

Total energy $W_{12}^{c}$ of the disclination system, shown in Fig. 6 can now easily found as

$W_{12}^{c}=E_{r 1}^{c}+E_{r 2}^{c}+E_{12}^{c}$.

Note that configurational forces acting on disclinations in the considered system are defined by the dependence of the total energy on the system parameters, but not of separate energy contributions.

\subsection{Wedge disclination in a two-phase cylinder}

For singular wedge disclination in a two-phase cylinder (this case is shown schematically in Fig. 7) boundary conditions of type A, see Section 2.2, must be fulfilled at the external cylinder surface, i.e. for $r=R_{2}$, and boundary conditions of type $\mathrm{C}$ at the interface inside a cylinder, i.e. for $r=R_{1}$.

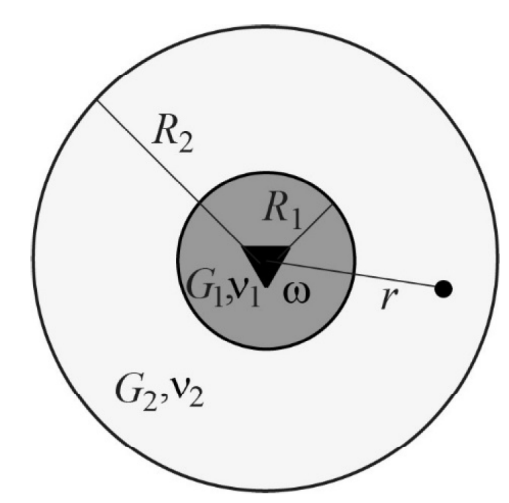

Fig. 7. Wedge disclination in a two-phase cylinder. In the domain $r \leq R_{1}$ elastically isotropic material is characterized by shear modulus $G_{1}$ and Poisson's ratio $v_{1}$ whereas for domain $R_{1} \leq r \leq R_{2}$ by shear modulus $G_{2}$ and Poisson's ratio $v_{2}$. The disclination line and Frank vector coincide with the cylinder axis. 
Solution for this problem was given in Ref. [28]. The stresses should be written separately for two domains: (i) for $r \leq R_{1}$ :

$$
\begin{aligned}
& \sigma_{r r}^{1}=\frac{G_{1} \omega}{2 \pi\left(1-v_{1}\right)}\left[\log \frac{r}{R_{1}}+\frac{2 G_{2}\left(1-v_{1}\right) R_{2}^{2}}{G_{2}\left(1-2 v_{1}\right)\left(R_{2}^{2}-R_{1}^{2}\right)+G_{1}\left(1-2 v_{2}\right) R_{1}^{2}+G_{1} R_{2}^{2}} \log \frac{R_{1}}{R_{2}}\right], \\
& \sigma_{\varphi \varphi}^{1}=\frac{G_{1} \omega}{2 \pi\left(1-v_{1}\right)}\left[1+\log \frac{r}{R_{1}}+\frac{2 G_{2}\left(1-v_{1}\right) R_{2}^{2}}{G_{2}\left(1-2 v_{1}\right)\left(R_{2}^{2}-R_{1}^{2}\right)+G_{1}\left(1-2 v_{2}\right) R_{1}^{2}+G_{1} R_{2}^{2}} \log \frac{R_{1}}{R_{2}}\right], \\
& \sigma_{z z}^{1}=v_{1}\left(\sigma_{r r}^{1}+\sigma_{\varphi \varphi}^{1}\right) ;
\end{aligned}
$$

(ii) for $R_{1} \leq r \leq R_{2}$ :

$$
\begin{aligned}
& \sigma_{r r}^{2}=\frac{G_{2} \omega}{2 \pi\left(1-v_{2}\right)}\left[\log \frac{r}{R_{2}}+\left(\frac{2 G_{1}\left(1-v_{2}\right) R_{2}^{2}}{G_{2}\left(1-2 v_{1}\right)\left(R_{2}^{2}-R_{1}^{2}\right)+G_{1}\left(1-2 v_{2}\right) R_{1}^{2}+G_{1} R_{2}^{2}}-1\right) \frac{\left(R_{2}^{2}-r^{2}\right) R_{1}^{2}}{\left(R_{2}^{2}-R_{1}^{2}\right) r^{2}} \log \frac{R_{1}}{R_{2}}\right], \\
& \sigma_{\varphi \varphi}^{2}=\frac{G_{2} \omega}{2 \pi\left(1-v_{2}\right)}\left[1+\log \frac{r}{R_{2}}+\left(1-\frac{2 G_{1}\left(1-v_{2}\right) R_{2}^{2}}{G_{2}\left(1-2 v_{1}\right)\left(R_{2}^{2}-R_{1}^{2}\right)+G_{1}\left(1-2 v_{2}\right) R_{1}^{2}+G_{1} R_{2}^{2}}\right) \frac{\left(R_{2}^{2}+r^{2}\right) R_{1}^{2}}{\left(R_{2}^{2}-R_{1}^{2}\right) r^{2}} \log \frac{R_{1}}{R_{2}}\right],
\end{aligned}
$$

$\sigma_{z z}^{2}=v_{2}\left(\sigma_{r r}^{2}+\sigma_{\varphi \varphi}^{2}\right)$

where $G_{1}, v_{1}$ and $G_{2}, v_{2}$ are shear moduli and Poisson's ratios for domains 1 and 2, correspondingly.

It is easy to check that the stresses defined by Eqs. (38) and (39) satisfy the boundary conditions mentioned above. In limit cases $G_{2}=0$ or $G_{2}=G_{1}=G, v_{2}=v_{1}=v$, these formulas reproduce the stresses for a wedge disclination in a uniform cylinder of radius $R_{1}$ or $R_{2}$, respectively, whereas with $G_{1}=0$ one gets the solution for hollow cylinder, see Eqs. (22). The other limit case for a disclination with rigid core can be obtained when assuming $G_{1}>>G_{2}$. This later case has been considered in detail and in other formulation in recent work [29].

The energy of a wedge disclination in two-phase cylinder is [28]:

$$
\begin{aligned}
& E_{0}^{c 2}=\frac{G_{2} \omega^{2} R_{2}^{2}}{16 \pi\left(1-v_{2}\right)}\left[1+\left(\frac{\left(1-v_{2}\right) G_{1}}{\left(1-v_{1}\right) G_{2}}-1\right) \frac{R_{1}^{2}}{R_{2}^{2}}+\right. \\
& \left.\left(\frac{2 G_{1}\left(1-v_{2}\right) R_{2}^{2}}{G_{2}\left(1-2 v_{1}\right)\left(R_{2}^{2}-R_{1}^{2}\right)+G_{1}\left(1-2 v_{2}\right) R_{1}^{2}+G_{1} R_{2}^{2}}-1\right) \frac{4 R_{1}^{2}}{\left(R_{2}^{2}-R_{1}^{2}\right)}\left(\log \frac{R_{1}}{R_{2}}\right)^{2}\right] .
\end{aligned}
$$

This formula transforms to Eq. (31) and Eq. (26) with $R_{1}=0$ and $G_{1}=0$, respectively.

The elastic behavior of wedge disclinations in various inhomogeneous structures of cylindrical symmetry was also considered. Authors of Refs. [30] and [31] studied the elastic interaction of wedge disclination dipoles with circular and annular inhomogeneities embedded in infinite matrix, respectively. The elasticity solution for a wedge disclination dipole interacting with a coated circular inhomogeneity in an infinite medium was found in Ref. [32].

Concluding this section, it makes sense to mention the analysis of the elastic behavior of wedge disclinations in a functionally graded cylinder recently performed in Ref. [33].

\section{PLANE ELASTICITY FOR DISCLINATIONS NEAR PLANAR INTERFACES}

\subsection{Wedge disclination at a free surface}

When a disclination approaches to the surface of a cylinder, e.g. disclination $\omega_{1}$ in Fig. 6 , the character of screening in the system changes: the distance to the nearest cylinder surface $d=R-r_{1}$ becomes the dominant screening parameter. In the limit $r_{1}, R \rightarrow \infty$ and $d=$ const we have a wedge disclination in a half-space. Airy stress function (defined in the coordinate system shown in Fig. 8) for such a disclination can be easily derived from Eq. (32): 


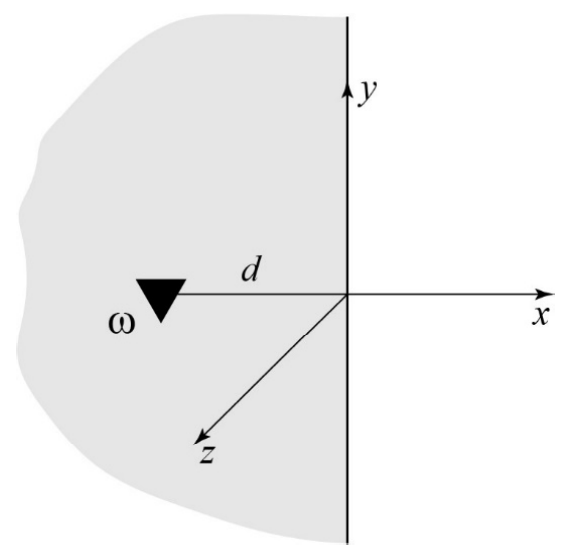

Fig. 8. Wedge disclination parallel to free the surface of a half-space. Disclination is at the distance $d$ from the free surface. The origin of Cartesian coordinate system $x, y, z$ is chosen to be at the surface.

$\chi^{d}=\frac{G \omega}{8 \pi(1-v)}\left((x+d)^{2}+y^{2}\right) \log \frac{(x+d)^{2}+y^{2}}{(x-d)^{2}+y^{2}}$,

where, as before, $\omega$ designates the disclination strength.

Exploring Eq. (41) together with Eqs. (17) leads to the stresses of the wedge disclination in a half-space:

$$
\begin{aligned}
& \sigma_{x x}=\frac{G \omega}{2 \pi(1-v)}\left[\frac{1}{2} \log \frac{(x+d)^{2}+y^{2}}{(x-d)^{2}+y^{2}}+\frac{y^{2}}{(x+d)^{2}+y^{2}}-\frac{y^{2}}{(x-d)^{2}+y^{2}}+2 x d \frac{y^{2}-(x-d)^{2}}{\left((x-d)^{2}+y^{2}\right)^{2}}\right], \\
& \sigma_{y y}=\frac{G \omega}{2 \pi(1-v)}\left[\frac{1}{2} \log \frac{(x+d)^{2}+y^{2}}{(x-d)^{2}+y^{2}}+\frac{(x+d)^{2}}{(x+d)^{2}+y^{2}}-\frac{(x-d)^{2}}{(x-d)^{2}+y^{2}}+2 d \frac{y^{2}(2 d-3 x)+(x-d)^{2}(2 d-x)}{\left((x-d)^{2}+y^{2}\right)^{2}}\right], \\
& \sigma_{z z}=v\left(\sigma_{x x}+\sigma_{y y}\right), \\
& \sigma_{x y}=\frac{G \omega}{2 \pi(1-v)}\left[\frac{(x-d) y}{(x-d)^{2}+y^{2}}-\frac{(x+d) y}{(x+d)^{2}+y^{2}}+2 y d \frac{y^{2}-x^{2}+d^{2}}{\left((x-d)^{2}+y^{2}\right)^{2}}\right] .
\end{aligned}
$$

In Refs. [34,35] the elastic fields of wedge disclination parallel to the surface of a half-space were found with different methods: using complex potentials [34] or with the virtual defect technique [35]. In the second method, to fulfill the boundary conditions for stresses on the free surface the following virtual defects were introduced: image wedge disclination of opposite sign placed at the point with coordinates $(d, 0)$ and the distribution of surface edge dislocations with their Burgers vector being parallel to the free surface [35]. It is assumed that the elastic fields of virtual defects have the physical meaning only for a domain where real wedge disclination is placed, i.e. for the halfspace $x \leq 0$. In the following it will be demonstrated that the virtual defect technique is effective in the solution of various boundary-value problems for disclinations. Here we only mention that the same approach was used to find elastic fields in an isotropic half-space not only for wedge disclinations, but also for straight-linear twist disclinations [36].

When analyzing the asymptotes of wedge disclination stresses at large distances from the free surface, i.e. $r=\sqrt{\left(x^{2}+y^{2}\right)}>>d$, the following scaling behavior was discovered [4,36]:

$$
\sigma_{i j} \sim \frac{G \omega}{1-v} \frac{d^{2}}{r^{2}},
$$

which is alike to the scaling law for the stresses of edge dislocations placed in the vicinity of a free surface. 


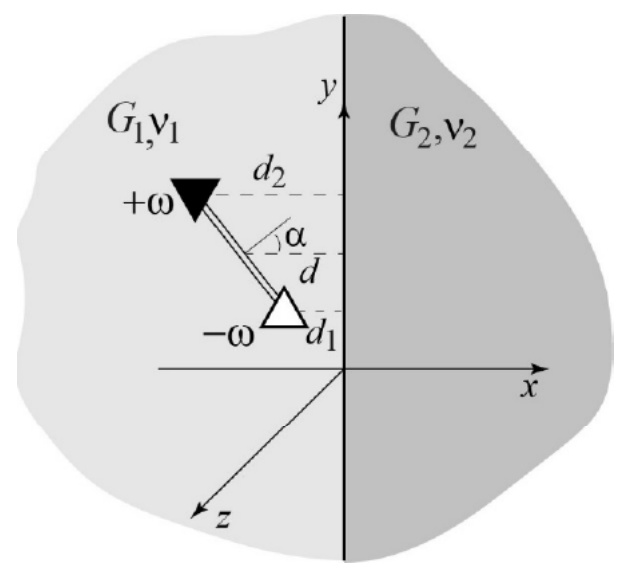

Fig. 9. Dipole of wedge disclinations near the internal boundary separating phases with different elastic properties.

Energies of isolated wedge disclination and their ensembles can be determined from Eqs. (35)-(37) by passing to the limit from disclinations in a cylinder to disclinations in a half-space as it is described in the beginning of this Section. This agrees with the results found in Refs. [35,37] by direct calculations based on Eqs. (22). In particular, for the energy $E^{d}$ per unit length of singular wedge disclination parallel to a free surface of elastically isotropic halfspace one finds:

$E^{d}=\frac{G \omega^{2} d^{2}}{4 \pi(1-v)}$.

Comparing the energies given by Eqs. (32) and (44) we note the same typical quadratic dependence on the parameter of screening $(R$ or $d$ ) and the different, as it should be expected, screening ability of free surfaces in cylinder in comparison to half-space: the wedge disclination energy surrounded by the surface, i.e. in a cylinder, is four times less for $R=d$. It is also natural that pair interaction of disclinations in a half-space demonstrates non-central character and depends on the distance for each of them to the surface [37]; the analysis of such interaction for the dipole configuration is briefly discussed in the next Section.

\subsection{Disclinations near interfaces}

In accordance with variants of boundary conditions mentioned in Section 2.2 we consider two cases: wedge disclinations in the vicinity of (i) phase boundary or (ii) sliding interface.

For the first case, we follow the results of Ref. [38] where the elastic properties of wedge disclination dipole in the geometry shown in Fig. 9 were investigated. It was found that for the case $\alpha=0$ (dipole arm being parallel to the phase boundary with $d$ being the dipole distance to the interface) Airy stress function that provides the fulfillment of the boundary conditions of type C (see Eqs. (8)) at the interface is written as

(i) for $x \leq 0$ :

$$
\begin{aligned}
\chi^{(1)}= & \frac{G_{1} \omega}{8 \pi\left(1-v_{1}\right)}\left[\left((x+d)^{2}+(l-y)^{2}\right) \log \left((x+d)^{2}+(l-y)^{2}\right)+\right. \\
& \left(\frac{B-A}{2}(x-d)^{2}-A(x+d)^{2}-\frac{A+B}{2}(l-y)^{2}\right) \log \left((x-d)^{2}+(l-y)^{2}\right)+ \\
& \left.\frac{A+B-2}{2}(l-y)^{2}+2(A-B)(x-d)(l-y) \tan ^{-1} \frac{l-y}{x-d}\right]_{l=d_{1}}^{l=d_{2}},
\end{aligned}
$$

(ii) for $x \geq 0$

$$
\begin{gathered}
\chi^{(2)}=\frac{G_{1} \omega}{8 \pi\left(1-v_{1}\right)}\left[\left(\frac{A-B}{2}\left(x^{2}-d^{2}\right)+\frac{A+B-2}{2}\left((x+d)^{2}+(l-y)^{2}\right)\right) \log \left((x+d)^{2}+(l-y)^{2}\right)+\right. \\
\left.\frac{A+B-2}{2}(l-y)^{2}+2(A-B)(x-d)(l-y) \tan ^{-1} \frac{l-y}{x+d}\right]\left.\right|_{l=d_{1}} ^{l=d_{2}},
\end{gathered}
$$


where $A$ and $B$ are Dundurs's coefficients [39]:

$$
A=\frac{G_{1}-G_{2}}{G_{1}-\left(3-4 v_{1}\right) G_{2}}, \quad B=\frac{\left(3-4 v_{2}\right) G_{1}-\left(3-4 v_{1}\right) G_{2}}{\left(3-4 v_{2}\right) G_{1}+G_{2}}
$$

with $G_{1}, v_{1}$ and $G_{2}, v_{2}$ being elastic moduli for materials 1 and 2, correspondingly, as shown in Fig. 9.

In the case of a free surface $(A=B=1), \chi^{(2)}$ vanishes and Eq. (45a) gives Airy stress function $\chi^{(1)}$ for wedge disclination dipole in elastically isotropic half-space. From Eqs. (45) one finds the stresses and other elastic fields that completely obey the boundary conditions of Eqs. (8).

For arbitrary combination of elastic constants, the elastic energy either for isolated wedge disclination or a disclination dipole in two joined half-spaces is infinite because the screening of the phase boundary is not effective enough and the elastic fields of such objects are not localized. Still, with the help of the results for stresses of wedge disclination dipole at a phase boundary or at a free surface configurational forces acting in such system can be determined. For example, the component of the force in the $x$-direction acting on the dipole shown in Fig. 9 is [38]:

$$
\begin{aligned}
& F_{x}^{\alpha}=\frac{G_{1} \omega^{2}}{4 \pi\left(1-v_{1}\right)}\left[( B - A ) \left(4 a \cos \alpha \tan ^{-1} \frac{a \cos \alpha}{d}+\right.\right. \\
& \left.\left.2 d \log \frac{d^{2}-a^{2} \cos ^{2} \alpha}{d^{2}+a^{2} \cos ^{2} \alpha}+2 a \sin \alpha \log \frac{d+a \sin \alpha}{d+a \sin \alpha}\right)+4 A \frac{d a^{2}}{d^{2}+a^{2} \cos ^{2} \alpha}\right],
\end{aligned}
$$

where $a$ is half of the dipole arm and the relation $l_{2}-l_{1}=2 a \cos \alpha$ is used. As it was explored for stresses, with $A=B=1$ we find the force attracting a wedge disclination dipole to the free surface:

$$
F_{x}^{d}=\frac{G \omega^{2}}{\pi(1-v)} \frac{d a^{2}}{d^{2}+a^{2} \cos ^{2} \alpha} .
$$

Interesting property of the force $F_{x}^{d}$ is the existence of the maximum in the dependence on $F_{x}^{d}$ distance at $d=a|\cos \alpha|$. The expression for the attractive force was first derived in Ref. [37], where the energy of wedge disclination dipole in a half-space was found:

$$
W^{d}=\frac{G \omega^{2} a^{2}}{2 \pi(1-v)}\left(2 \sin ^{2} \alpha+\log \frac{d^{2}+a^{2} \cos ^{2} \alpha}{a^{2}}\right) .
$$

This expression permits also to determine the configurational momentum $M^{d}$ :

$$
M^{d}=-\frac{\partial W^{d}}{\partial \alpha}=-\frac{G \omega^{2} a^{2}}{2 \pi(1-v)} \frac{2 d^{2}+2 a^{2} \cos ^{2} \alpha-a^{2}}{d^{2}+a^{2} \cos ^{2} \alpha} \sin 2 \alpha,
$$

$\lim _{d \gg a} M^{d}=-\frac{G \omega^{2} a^{2}}{\pi(1-v)} \sin 2 \alpha$.

which tends to bring the dipole in the orientation with minimum of energy with $\alpha=0$. For this orientation one can analyze the interaction between disclinations when they are in the dipole configuration. The force for this interaction is [37]:

$$
F_{a}^{d}=-\frac{\partial W^{d}}{\partial(2 a)}=\frac{G \omega^{2} a}{2 \pi(1-v)}\left(\frac{d^{2}}{a^{2}+d^{2}}+\log \frac{a^{2}}{a^{2}+d^{2}}\right) .
$$

It is easy to check that force $F_{x}^{d}$ is always attractive and reaches the maximum value for $\alpha \approx 0.3 \mathrm{~d}$.

The second example deals with the interaction of disclinations with so-called sliding boundaries. It is assumed that there exists an internal surface that does not support shear forces. This also means that shear stresses vanish on such an interface. The analysis of the behavior of wedge disclinations placed in the vicinity of planar sliding boundary of infinite or finite (as given in Fig. 10) extent was performed in Ref. [40]. It is worth to note that here the well-posed elasticity problem was not formulated, instead the problem of the equilibrium of distributed edge dislocations in the field of the wedge disclination was investigated assuming such dislocations as carriers of sliding. 


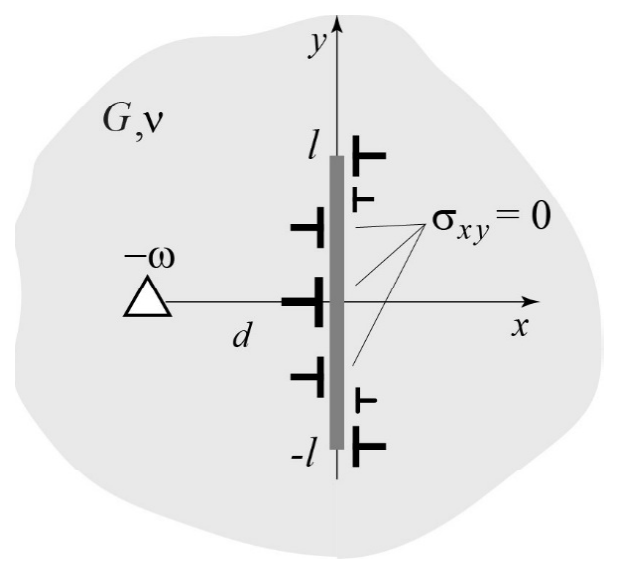

Fig. 10. Negative wedge disclination near the sliding boundary. Virtual edge dislocations responsible for sliding and vanishing shear stresses in the selected part of the interface.

Negative wedge disclination in the geometry shown in Fig. 10 generates the following shear stresses in the plane of the boundary:

$\sigma_{x y}=\frac{G \omega}{2 \pi(1-v)} \frac{d y}{d^{2}+y^{2}}$.

The pileup of edge dislocations with their Burgers vector being parallel to $y$-axis will completely compensate these stresses in the region of the interface $-l<y<+l$ if their distribution function $f^{l}(y)$ (the dislocation density per the length of the interface in $y$-direction) is:

$f^{l}(y)=\frac{\omega}{\pi b} \frac{d}{\sqrt{l^{2}-y^{2}}}\left(\frac{d \sqrt{l^{2}+d^{2}}}{y^{2}+d^{2}}-1\right) ;$

where $b$ is the infinitesimally small magnitude of virtual dislocation Burgers vector. For the sliding boundary of infinite extent when $l \rightarrow \infty$ the distribution function acquires the simpler form:

$f^{\infty}(y)=\frac{\omega}{\pi b} \frac{d^{2}}{y^{2}+d^{2}}$.

With the help of the found distribution functions given by Eqs. (52) or (53) one can find the stresses of the wedge disclination (or disclination configurations) placed in the material with sliding boundary, which will be just superposition of the stress filed of the disclinations in infinite media and stress field induced by virtual edge dislocation pileup [40]. For example, for the shear stress component that is of the most interest in the case of infinite sliding boundary we have:

$$
\begin{aligned}
& \sigma_{x y}=\frac{G \omega}{2 \pi(1-v)} \times \\
& {\left[\frac{(x+d) y}{(x+d)^{2}+y^{2}}+\frac{y d\left(x^{2}-y^{2}-d^{2}\right)}{\left((|x|+d)^{2}+y^{2}\right)^{2}}\right],}
\end{aligned}
$$

which obviously demonstrates the fulfillment of the condition $\sigma_{x y}(x=0, y) \equiv 0$.

The interaction of wedge disclination dipoles with sliding boundary demonstrates an unexpected feature related to the dipole arm orientation with respect to the boundary, see Refs. [40] and [4]. For two important dipole orientations (parallel and normal to the boundary) for the forces we have:

$F_{x}^{\infty \|}=\frac{G \omega^{2}}{2 \pi(1-v)} d\left(\log \frac{a^{2}+d^{2}}{d^{2}}-\frac{a^{2}}{a^{2}+d^{2}}\right)$,

$$
\begin{aligned}
& F_{x}^{\infty \perp}=\frac{G \omega^{2}}{2 \pi(1-v)} d \times \\
& \left(\log \frac{d^{2}}{d^{2}-a^{2}}-\frac{a}{d} \log \frac{d-a}{d+a}-\frac{a^{2}}{d^{2}}\right), d>a .
\end{aligned}
$$

The analysis of Eqs. (54) shows that the dipoles having an arm parallel to the infinite sliding boundary will repel from it $\left(F_{x}^{\infty}<0\right)$ whereas the dipoles having a perpendicular arm will be attracted $\left(F_{x}^{\infty \perp}>0\right)$ to such an interface.

\subsection{Disclinations in the plate of a finite thickness}

For the sources of internal stresses, e.g. dipole of wedge disclinations placed with arm $2 a$ in a plate of finite thickness $2 t$ (see Fig. 11), boundary conditions of the type defined by Eq. (5) must be met for the both surfaces, i.e. for $x= \pm t$. The other parameters include positions $\left(x_{1}, y_{1}\right)$ and $\left(x_{2}, y_{2}\right)$ of positive $+\omega$ and negative $-\omega$ disclinations, respectively. This boundary-value problem can be solved as it was proposed in Ref. [41] by finding distribution functions $f^{k}(y), k=1+, 2+, 1-, 2$ - of virtual surface edge dislocations, which are placed at the corresponding surface of the plate and possess Burgers vector in the directions being either parallel or perpendicular to the surfaces of the plate. It appears that in this case, one cannot determine the distribution functions in the analytical form (to the contrary to the case considered in the Section 4.2), but only their Fourier transforms $\hat{f}^{k}(s)^{1}$. Then, the stresses of a disclination dipole in a plate can be found in the form of single integrals of $\hat{f}^{k}(s)$ :

\footnotetext{
${ }^{1}$ We define the pair of Fourier transforms as:

$\hat{\psi}(s)=1 /(\sqrt{2 \pi}) \int_{-\infty}^{\infty} \psi(y) \exp (-i s y) \mathrm{d} y$ and $\psi(y)=(1 / \sqrt{2 \pi}) \int_{-\infty}^{\infty} \hat{\psi}(y) \exp ($ is $y) \mathrm{d} s$.
} 


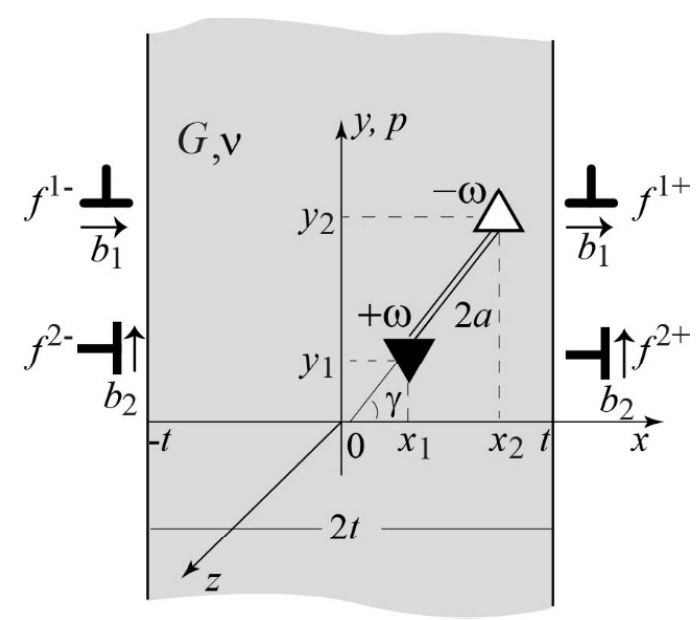

Fig. 11. Wedge disclination dipole in a plate of finite thickness. The line of disclinations are parallel to the plate surfaces; $f^{1,2 \pm}$ are distribution functions of virtual edge dislocations, which are introduced to fulfil boundary conditions.

$$
\sigma_{i j}^{t}(x, y)=\sigma_{i j}^{\infty}(x, y)+\int_{-\infty}^{+\infty} \sum_{k} \hat{f}^{k}(s) \hat{\sigma}_{i j}^{k}(x, s) \exp (i s y) \mathrm{d} s \quad(k=1+, 1-, 2+, 2-),
$$

where $\sigma_{i j}^{\infty}(x, y)$ is the field of a disclination in infinite medium (see Eqs. (17)), $\hat{\sigma}_{i j}^{k}(x, s)$ is the Fourier transform of the stresses of a probe dislocation in the $k$-th array.

Analytic expressions for the Fourier transforms included in relation (55) were obtained in Refs. [38,39], they look like:

$$
\begin{aligned}
& \hat{f}^{1+}(s)=i \operatorname{sgn}(s) \frac{\omega t}{\sqrt{2 \pi} b H} \times \\
& {\left[\left(\frac{R_{1}}{\mid \tilde{s}_{\mid}}+\tilde{u}_{2-} R_{2}\right) \exp \left(-\tilde{u}_{2-}|\tilde{s}|-i \tilde{y}_{2} \tilde{s}\right)-\left(\frac{R_{1}}{|\tilde{s}|}+\tilde{u}_{1-} R_{2}\right) \exp \left(-\tilde{u}_{1-}|\tilde{s}|-i \tilde{y}_{1} \tilde{s}\right)+\right.} \\
& \left.\left(\frac{R_{3}}{|\tilde{s}|}+\tilde{u}_{2+} R_{4}\right) \exp \left(-\tilde{u}_{2+}|\tilde{s}|-i \tilde{y}_{2} \tilde{s}\right)-\left(\frac{R_{3}}{|\tilde{s}|}+\tilde{u}_{1+} R_{4}\right) \exp \left(-\tilde{u}_{1+}|\tilde{s}|-i \tilde{y}_{1} \tilde{s}\right)\right] \\
& \hat{f}^{1-}(s)=i \operatorname{sgn}(s) \frac{\omega t}{\sqrt{2 \pi b H}} \times \\
& {\left[\left(\frac{R_{3}}{|\tilde{s}|}+\tilde{u}_{2-} R_{4}\right) \exp \left(-\tilde{u}_{2-}|\tilde{s}|-i \tilde{y}_{2} \tilde{s}\right)-\left(\frac{R_{3}}{|\tilde{s}|}+\tilde{u}_{1-} R_{4}\right) \exp \left(-\tilde{u}_{1-}|\tilde{s}|-i \tilde{y}_{1} \tilde{s}\right)+\right.} \\
& \left.\left(\frac{R_{1}}{|\tilde{s}|}+\tilde{u}_{2+} R_{2}\right) \exp \left(-\tilde{u}_{2+}|\tilde{s}|-i \tilde{y}_{2} \tilde{s}\right)-\left(\frac{R_{1}}{|\tilde{s}|}+\tilde{u}_{1+} R_{4}\right) \exp \left(-\tilde{u}_{1+}|\tilde{s}|-i \tilde{y}_{1} \tilde{s}\right)\right] \text {, } \\
& \hat{f}^{2+}(s)=\frac{\omega t}{\sqrt{2 \pi} b H} \times \\
& {\left[\left(\frac{T_{1}}{|\tilde{s}|}+\tilde{u}_{2-} T_{2}\right) \exp \left(-\tilde{u}_{2-}|\tilde{s}|-i \tilde{y}_{2} \tilde{s}\right)-\left(\frac{T_{1}}{|\tilde{s}|}+\tilde{u}_{1-} T_{2}\right) \exp \left(-\tilde{u}_{1-}|\tilde{s}|-i \tilde{y}_{1} \tilde{s}\right)+\right.} \\
& \left.\left(\frac{T_{3}}{|\tilde{s}|}+\tilde{u}_{2+} T_{4}\right) \exp \left(-\tilde{u}_{2+}|\tilde{s}|-i \tilde{y}_{2} \tilde{s}\right)-\left(\frac{T_{3}}{|\tilde{s}|}+\tilde{u}_{1+} T_{4}\right) \exp \left(-\tilde{u}_{1+}|\tilde{s}|-i \tilde{y}_{1} \tilde{s}\right)\right] \text {, } \\
& \hat{f}^{2-}(s)=-\frac{\omega t}{\sqrt{2 \pi b H}} \times \\
& {\left[\left(\frac{T_{3}}{\mid \tilde{s}_{\mid}}+\tilde{u}_{2-} T_{4}\right) \exp \left(-\tilde{u}_{2-}|\tilde{s}|-i \tilde{y}_{2} \tilde{s}\right)-\left(\frac{T_{3}}{|\tilde{s}|}+\tilde{u}_{1-} T_{4}\right) \exp \left(-\tilde{u}_{1-}|\tilde{s}|-i \tilde{y}_{1} \tilde{s}\right)+\right.} \\
& \left.\left(\frac{T_{1}}{|\tilde{s}|}+\tilde{u}_{2+} T_{2}\right) \exp \left(-\tilde{u}_{2+}|\tilde{s}|-i \tilde{y}_{2} \tilde{s}\right)-\left(\frac{T_{1}}{|\tilde{s}|}+\tilde{u}_{1+} T_{4}\right) \exp \left(-\tilde{u}_{1+}|\tilde{s}|-i \tilde{y}_{1} \tilde{s}\right)\right]
\end{aligned}
$$


$\hat{\sigma}_{x x}^{1+,-}(s)=i \operatorname{sgn}(s) \frac{G b}{2 \sqrt{2 \pi}(1-v)}\left(\left(1+|s| u_{-,+}\right) \exp \left(-|s| u_{-,+}\right)\right)$,

$\hat{\sigma}_{y y}^{1+,-}(s)=i \operatorname{sgn}(s) \frac{G b}{2 \sqrt{2 \pi}(1-v)}\left(\left(1-|s| u_{-,+}\right) \exp \left(-|s| u_{-,+}\right)\right)$,

$\hat{\sigma}_{x y}^{1+,-}(s)=\mp \frac{G b}{2 \sqrt{2 \pi}(1-v)}\left(|s| u_{-,+} \exp \left(-|s| u_{-,+}\right)\right)$,

$\hat{\sigma}_{x x}^{2+,-}(s)=\hat{\sigma}_{x y}^{1+,-}(s)$,

${\sigma_{y y}^{2+,-}}_{y y}(s)=\frac{G b}{2 \sqrt{2 \pi}(1-v)} \exp \left(-|s| u_{-,+}\right)$,

$\bar{\sigma}_{x y}^{2+,-}(s)=\widehat{\sigma}_{y y}^{1+,-}(s)$,

where $\omega$ is disclination strength, $b$ is the magnitude of dislocation Burgers vector, $G$ is the shear modulus, $v$ is the Poisson's ratio;

$u_{+,-}=t \pm x, \tilde{s}=s t, \quad \tilde{u}_{1+,-}=\left(t \pm x_{1}\right) / t, \quad \tilde{u}_{2+,-}=\left(t \pm x_{2}\right) / t, \quad \tilde{y}_{1}=y_{1} / t, \quad \tilde{y}_{2}=y_{2} / t ; \quad H=\exp (4 \tilde{s})+$

$\exp (-4 \tilde{s})-16 \tilde{s}^{2}-2, R_{1}=\exp (4|\tilde{s}|)-8 \tilde{s}^{2}+4|\tilde{s}|-1, R_{2}=\exp (4|\tilde{s}|)+4|\tilde{s}|-1, R_{3}=\exp (-2|\tilde{s}|)-$

$\exp (2|\tilde{s}|)-2|\tilde{s}|(\exp (-2|\tilde{s}|)+\exp (2|\tilde{s}|)), R_{4}=\exp (-2|\tilde{s}|)-\exp (2|\tilde{s}|)-4|\tilde{s}| \exp (2|\tilde{s}|), T_{1}=8 \tilde{s}^{2}$,

$T_{2}=\exp (4|\tilde{s}|)-4|\tilde{s}|-1, T_{3}=2|\tilde{s}| \exp (-2|\tilde{s}|)-2|\tilde{s}| \exp (2|\tilde{s}|)$

$T_{4}=\exp (2|\tilde{s}|)-4|\tilde{s}| \exp (2|\tilde{s}|)-\exp (-2|\tilde{s}|)$.

The energy $E_{t}^{d d}$ of the disclination dipole in the plate of finite thickness can be found by calculating the mechanical work done in the process of the dipole formation or by applying general formulas of Eqs. (22a and 22b). The first from the mentioned techniques leads to the following expression [41]:

$E_{t}^{d d}=\frac{1}{2} \omega \sum_{m=1}^{2}(-1)^{m-1}\left[\int_{x_{m}}^{t}\left(x-x_{m}\right)\left(\sigma_{i j}^{\infty}\left(x, y_{m}\right)+\int_{-\infty}^{+\infty} \sum_{k} \hat{f}^{k}(s) \hat{\sigma}_{i j}^{k}(x, s) \exp \left(i s y_{m}\right) \mathrm{d} s\right) \mathrm{d} x\right]$,

where $m$ is the number for the disclinations in the dipole: $m=1$ for positive wedge disclination and $m=2$ for negative one, correspondingly.

Analysis, see Refs. $[4,41]$ shows that the integrations in Eqs. (55) and (58) in the general case of the plate of finite thickness cannot be performed analytically. However, it has been demonstrated in Ref. [42] that with the help of the obtained formulas and using passages to the limits, e.g., when plate thickness tends to the infinity with disclination dipole being kept near one of the plate surfaces, the analytical relations (given is Section 4.1) for wedge disclinations parallel to a free surface of elastic half-space are reproduced exactly.

Other limiting cases allow to investigate elastic properties of a single wedge disclination in the plate of finite thickness. For this one has to assume that either one of the disclinations of the dipole comes to the surface of a plate, i.e., $x_{2}= \pm t$, or its ordinate tends to infinity, i.e. $y_{2} \rightarrow \pm \infty$. Then, the relations found for the stresses of a dipole, Eq. (55) give the stress field of an isolated wedge disclination in a plate. Comparison of stress distributions for wedge disclinations with various character of screening: in a cylinder, at a free surface, or in a plate shows that in a vicinity of disclination line the stresses are only slightly affected by boundary conditions; for example, in the core of a positive disclination there is always compression. However, the position of neutrals (the contours at which the stresses change the sign) and their configurations are uniquely determined by the body shape and the location of the disclination in the body as it can be seen from the stress maps collected in Ref. [4].

The self-energy of a disclination in a plate $E_{t}^{d}$ can be calculated with the help of formula like Eq. (58). The energy is maximal when disclination is placed in the center of a plate [41]:

$$
E_{t}^{d} \cong 0.182 \frac{G \omega^{2} t^{2}}{2 \pi(1-v)} .
$$




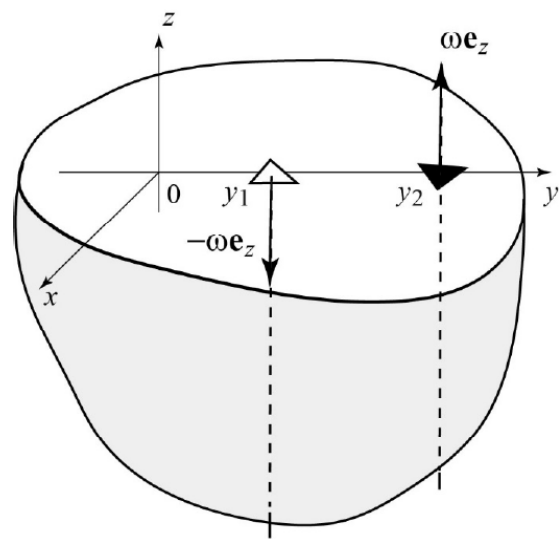

Fig. 12. Dipole of wedge disclinations with the lines of defects being normal to the surface of a half-space.

Comparing Eqs. (59), (44), and (32) one concludes that the energy for a disclination sitting in the plate is between the energies of a wedge disclination in a cylinder and one in a half-space when $R=t=d$. This obviously means that among plane elasticity problem cylinder possesses the highest screening ability for wedge disclinations.

Presence of wedge disclinations in a plate induces another phenomenon, namely plate bending [4,41]. The straightforward method to find the bending angle $\theta$ of the plate that contains a wedge disclination dipole, is to explore the work reciprocity principle (WRP), for details on WRP see Ref. [11]. According to WRP, the work of the momentum with component $M_{z}$ (in the geometry of Fig. 11) required to bend the plate by the angle $\theta$ is equal to that of normal bending stresses $\sigma_{y y}^{M_{z}^{z}}$ when forming disclination dipole in the plate:

$M_{z} \theta=\int_{-\infty}^{+\infty} \mathrm{d} z\left(\int_{x_{1}}^{t} \omega \sigma_{y y}^{M_{z}}(x)\left(x-x_{1}\right) \mathrm{d} x-\int_{x_{2}}^{t} \omega \sigma_{y y}^{M_{z}}(x)\left(x-x_{2}\right) \mathrm{d} x\right)$,

where $\sigma_{y y}^{M_{z}}=M_{z} x / I_{z}$ with $I_{z}$ being a component of the momentum of inertia of the plate cross-section. Then, from Eq. (60) for angle $\theta$ we find:

$\theta=\frac{3}{2} \omega \frac{x_{2}-x_{1}}{t}\left(1-\frac{x_{1}^{2}+x_{1} x_{2}+x_{2}^{2}}{3 t^{2}}\right)$.

Interesting and important observation follows form Eq. (61) regarding the bending of the plate with a single disclination; the bending angle can be determined when one of disclinations of the dipole being placed on one of the plate surfaces. Unlike the elastic fields that angle $\theta$ will depend on the procedure of the disclination formation in the plate and therefore cannot be found explicitly basing only on the position of a single disclination in the plate.

\section{3D ELASTICITY BOUNDARY-VALUE PROBLEMS FOR WEDGE DISCLINATIONS}

In this section, we present results for the solution of spatial elasticity boundary-value problems for wedge disclinations. The necessity of solving of such class of problems is dictated by a possible variation of the materials properties along the disclination lines.

\subsection{Disclinations normal to the surface of a half-space}

To find elastic fields of wedge disclinations normal to the free surface of a half-space it is convenient to consider again a dipole configuration (Fig. 12), in which the positions of disclinations in chosen co-ordinate system are given by parameters $y_{1}$ and $y_{2}$. As in the previous cases to satisfy the boundary conditions at the free surface set by Eq. (5), the stresses $\sigma_{i j}^{n}$ of the dipole of wedge disclinations normally emerging the surface can be written as the sum of stresses of the dipole in infinite medium $\sigma_{i j}^{\infty}$ and additional field $\sigma_{i j}^{i}$ that needs to be determined:

$\sigma_{i j}^{n}(x, y, z)=\sigma_{i j}^{\infty}(x, y)+\sigma_{i j}^{i}(x, y, z)$.

In Ref. [43] the solution to the considered problem was found with the help of harmonic three dimensional stress-functions [11]. In particular, additional stresses for disclination dipole were obtained: 


$$
\begin{aligned}
& \sigma_{x x}^{i}=\left.\frac{G \omega v}{2 \pi(1-v)}\left(-(1+2 v) \log \left(r_{z}-z\right)+2 z \frac{r_{z}\left(r_{z}-z\right)-x^{2}}{r_{z}\left(r_{z}-z\right)^{2}}-(1-2 v) \frac{z\left(r_{z}-z\right)-x^{2}}{\left(r_{z}-z\right)^{2}}\right)\right|_{\xi=y_{1}} ^{\xi=y_{2}}, \\
& \sigma_{y y}^{i}=\left.\frac{G \omega v}{2 \pi(1-v)}\left(-(1+2 v) \log \left(r_{z}-z\right)+2 z \frac{r_{z}\left(r_{z}-z\right)-(\xi-y)^{2}}{r_{z}\left(r_{z}-z\right)^{2}}-(1-2 v) \frac{z(r-z)-(\xi-y)^{2}}{\left(r_{z}-z\right)^{2}}\right)\right|_{\xi=y_{1}} ^{\xi=y_{2}}, \\
& \sigma_{z z}^{i}=\left.\frac{G \omega v}{2 \pi(1-v)}\left(-2 \log \left(r_{z}-z\right)+2 \frac{z}{r_{z}}\right)\right|_{\xi=y_{1}} ^{\xi=y_{2}} \\
& \sigma_{x y}^{i}=\left.\frac{G \omega v}{2 \pi(1-v)} \frac{x(\xi-y)\left(2 z+(1-2 v) r_{z}\right)}{r_{z}\left(r_{z}-z\right)^{2}}\right|_{\xi=y_{1}} ^{\xi=y_{2}} \\
& \sigma_{y z}^{i}=-\left.\frac{G \omega v}{2 \pi(1-v)} \frac{x z}{r_{z}\left(r_{z}-z\right)^{2}}\right|_{\xi=y_{1}} ^{\xi \xi=y_{2}}, \\
& \sigma_{y z}^{i}=\left.\frac{G \omega v}{2 \pi(1-v)} \frac{(y-\xi) z}{r_{z}\left(r_{z}-z\right)^{2}}\right|_{\xi=y_{1}} ^{\xi=y_{2}},
\end{aligned}
$$

where $r_{z}^{2}=x^{2}+(\xi-y)^{2}+z^{2}$. Simple analysis shows that boundary conditions for stress components defined by Eq. (62) are satisfied identically, i.e. $\left.\sigma_{z z}^{n}(x, y, z)\right|_{z=0} \equiv 0,\left.\sigma_{y z}^{n}(x, y, z)\right|_{z=0} \equiv 0,\left.\sigma_{z x}^{n}(x, y, z)\right|_{z=0} \equiv 0$.

In general, the fields for a single wedge disclination that is normal to a free surface differ significantly from those of a defect in an infinite medium. For example, from Eqs. (18), (62), and (63) for the stress component $\sigma_{z z}^{n 1}$ of an isolated wedge disclination one can get:

$$
\sigma_{z z}^{n 1}(x, y, z)=-\frac{2 G \omega}{3 \pi(1-v)} \frac{z^{3}}{r^{3}}, \text { for } r \gg z,
$$

where as before $r^{2}=x^{2}+y^{2}$. Unlike to the dependence given by Eq. (18c) this component does not diverge as function of $r$; by contrast, it demonstrates fast decay. However, the components $\sigma_{x x}^{n 1}$ and $\sigma_{y y}^{n 1}$ maintain their logarithmic dependence on $r$. This fact and the dependence of elastic fields on co-ordinate $z$ do not allow to define an energy of wedge disclination per unit length.

\subsection{Wedge disclinations in the plate of a finite thickness}

To find the stresses of wedge disclinations being perpendicular to the surfaces of a plate (see Fig. 13, where dipole configuration is shown) the technique of virtual dislocation loops can be explored $[15,44,45]$. Utilizing this technique, the families of prismatic dislocation loops and loops of radial Somigliana dislocations with distribution functions ${ }^{ \pm} f_{1,2}^{p}(c)$ and ${ }^{ \pm} f_{1,2}^{s}(c)$, respectively, are introduced for two surfaces of a plate. In the adopted in Fig. 13 notations the upper left index ( + or -) designates the side of a plate, the upper right one ( $p$ or $s$ ) is responsible for the type of virtual loops and the subscript ( 1 or 2 ) is anchored to the disclination in a dipole, whereas $c$ is a variable radius of the loops, and $2 t$ is the thickness of a plate.

Then, the stress field of wedge disclination dipole in the plate $\sigma_{i j}^{t}(r, \varphi, z)$ is represented as a sum of the dipole filed in an infinite elastic medium $\sigma_{i j}^{\infty}(r)$ and the field $\sigma_{i j}^{a}(r, \varphi, z)$ generated by the distributions of virtual circular dislocation loops:

$$
\sigma_{i j}^{t}(r, \varphi, z)=\sigma_{i j}^{\infty}(r)+\sigma_{i j}^{a}(r, \varphi, z)
$$

where cylindrical coordinates are utilized. Note that similar representations were used in Eqs. (55) and (62). 


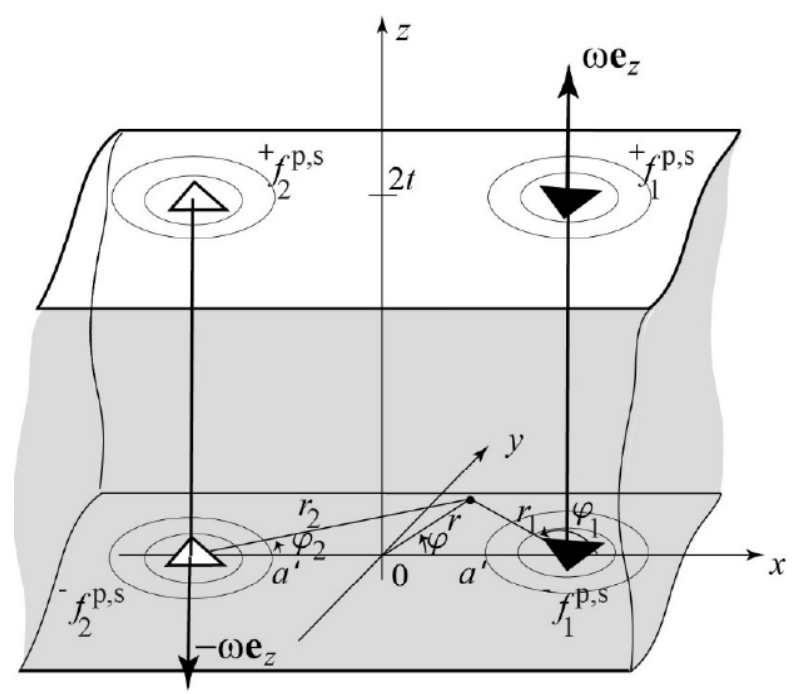

Fig. 13. Dipole of wedge disclinations whose line are normal to the surface of the plate of finite thickness. To meet boundary conditions distributions of prismatic dislocation loops ${ }^{ \pm} f_{1,2}^{p}$ and radial Somigliana dislocation loops ${ }^{ \pm} f_{1,2}^{s}$ are introduced.

Boundary conditions of Eq. (5) written with the help of Eq. (65) in terms of the above introduced distribution functions of loop defects constitute the system of integral equations that can be solved by exploring the technique of integral transformation. In contrast to the case considered in Section 4.3 where for disclination lines parallel to plate surfaces the Fourier transform is used, here for the solution the application of Hankel-Bessel integral transformation $^{2}$ becomes effective. It was found in Ref. [15] that Hankel transform ${ }^{ \pm} H_{1,2}$ of the dislocation loop distribution functions ${ }^{ \pm} f^{p, s}(c)$ (here we omit subscripts because of the symmetry of the problem)

$$
\begin{aligned}
& { }^{ \pm} H_{1}(\beta)=\int_{0}^{\infty} J_{1}(\beta c)^{ \pm} f^{p}(c) c \mathrm{~d} c, \\
& { }^{ \pm} H_{2}(\beta)=\int_{0}^{\infty} J_{2}(\beta c)\left({ }^{ \pm} f^{s}(c) c\right) c \mathrm{~d} c
\end{aligned}
$$

can be found explicitly in analytical form:

$$
\begin{aligned}
& H_{1}={ }^{-} H_{1}\left(\frac{\pi b}{\omega v}\right)={ }^{+} H_{1}\left(\frac{\pi b}{\omega v}\right)=\frac{2}{\beta^{2}} \frac{(1-2 t \beta) \exp (-2 t \beta)-1}{1+(4 t \beta-\exp (-2 t \beta)) \exp (-2 t \beta)}, \\
& H_{2}={ }^{-} H_{1}\left(\frac{\pi}{v}\right)=-{ }^{+} H_{2}\left(\frac{\pi}{v}\right)=-\frac{4}{\beta} \frac{t \exp (-2 t \beta)}{1+(4 t \beta-\exp (-2 t \beta)) \exp (-2 t \beta)}
\end{aligned}
$$

In Eqs. (66) and below in the text $J_{n}(\varsigma)$ are Bessel functions of argument $\varsigma$ and $n=0,1,2 \ldots$

As for the case of disclinations with parallel orientation of their lines with respect to plate surface (see Eq. (55)), to find the stresses of virtual dislocation loops there is no need to have distribution functions themselves, but it is enough to work with their Hankel transforms given by Eqs. (67). Such an approach leads to the following formulas for stresses $\sigma_{i j}^{a}(r, \mathrm{j}, z)[4,15]$ :

$$
\begin{aligned}
& \sigma_{r r}^{a}=\frac{G \omega v}{4 \pi(1-v)} \sum_{k=1}^{2}(-1)^{k-1}\left[\int_{0}^{\infty} J_{0}\left(r_{k} \beta\right)\left(\left(2 S_{1}+S_{2}\right) H_{1}+\left(2 S_{3}+S_{4}\right) H_{2}\right) \beta \mathrm{d} \beta+\right. \\
& \left.\quad \cos 2\left(\varphi-\varphi_{k}\right) \int_{0}^{\infty} J_{2}\left(r_{k} \beta\right)\left(S_{2} H_{1}+S_{4} H_{2}\right) \beta \mathrm{d} \beta\right],
\end{aligned}
$$

${ }^{2} \mathrm{~A}$ pair of Hankel-Bessel integral transforms are defined as: $H_{\mathrm{v}}(\lambda)=\int_{0}^{\infty} f(r) J_{\mathrm{v}}(\lambda r) r \mathrm{~d} r$ and $f(r)=\int_{0}^{\infty} H_{v}(\lambda) J_{v}(\lambda r) \lambda \mathrm{d} \lambda[46]$. 


$$
\begin{aligned}
& \sigma_{\varphi \varphi}^{a}=\frac{G \omega v}{4 \pi(1-v)} \sum_{k=1}^{2}(-1)^{k-1}\left[\int_{0}^{\infty} J_{0}\left(r_{k} \beta\right)\left(\left(2 S_{1}+S_{2}\right) H_{1}+\left(2 S_{3}+S_{4}\right) H_{2}\right) \beta \mathrm{d} \beta-\right. \\
& \left.\cos 2\left(\varphi-\varphi_{k}\right) \int_{0}^{\infty} J_{2}\left(r_{k} \beta\right)\left(S_{2} H_{1}+S_{4} H_{2}\right) \beta \mathrm{d} \beta\right], \\
& \sigma_{z z}^{a}=\frac{G \omega v}{2 \pi(1-v)} \sum_{k=1}^{2}(-1)^{k-1} \int_{0}^{\infty} J_{0}\left(r_{k} \beta\right)\left(T_{1} H_{1}+T_{2} H_{2}\right) \beta \mathrm{d} \beta, \\
& \sigma_{r \varphi}^{a}=-\frac{G \omega v}{4 \pi(1-v)} \sum_{k=1}^{2}(-1)^{k-1} \sin 2\left(\varphi_{k}-\varphi\right) \int_{0}^{\infty} J_{2}\left(r_{k} \beta\right)\left(S_{2} H_{1}+S_{4} H_{2}\right) \beta \mathrm{d} \beta, \\
& \sigma_{z \varphi}^{a}=\frac{G \omega v}{2 \pi(1-v)} \sum_{k=1}^{2}(-1)^{k-1} \sin 2\left(\varphi_{k}-\varphi\right) \int_{0}^{\infty} J_{1}\left(r_{k} \beta\right)\left(Q_{1} H_{1}+Q_{2} H_{2}\right) \beta \mathrm{d} \beta, \\
& \sigma_{z r}^{a}=\frac{G \omega v}{2 \pi(1-v)} \sum_{k=1}^{2}(-1)^{k-1} \sin 2\left(\varphi_{k}-\varphi\right) \int_{0}^{\infty} J_{1}\left(r_{k} \beta\right)\left(Q_{1} H_{1}+Q_{2} H_{2}\right) \beta \mathrm{d} \beta,
\end{aligned}
$$

where the meaning of $r_{k}$ and $\varphi_{k}$ (with $k=1.2$ ) becomes clear from the schematics in Fig. 13 (note that these parameters can be expressed via polar coordinates $(r, \varphi)$ and half of the dipole arm $\left.a^{\prime}\right)$ and the following designations are introduced:

$$
\begin{aligned}
& S_{1}=(-1+z \beta) \exp (-z \beta)-(1+(z-2 t) \beta) \exp ((z-2 t) \beta), \\
& S_{2}=(1-2 v-z \beta) \exp (-z \beta)+(1-2 v+(z-2 t) \beta) \exp ((z-2 t) \beta), \\
& S_{3}=(2-z \beta) \exp (-z \beta)+(2+(z-2 t) \beta) \exp ((z-2 t) \beta), \\
& S_{4}=(-2+2 v+z \beta) \exp (-z \beta)-(2-2 v+(z-2 t) \beta) \exp ((z-2 t) \beta), \\
& T_{1}=-(1+z \beta) \exp (-z \beta)-(1-(z-2 t) \beta) \exp ((z-2 t) \beta), \\
& T_{2}=z \beta \exp (-z \beta)-(z-2 t) \beta \exp ((z-2 t) \beta), \\
& Q_{1}=-z \beta \exp (-z \beta)-(z-2 t) \beta \exp ((z-2 t) \beta), \\
& Q_{2}=(-1+z \beta) \exp (-z \beta)+(1+(z-2 t) \beta) \exp ((z-2 t) \beta) .
\end{aligned}
$$

The found stresses of wedge disclination dipole in a plate can be tested for fulfillment of boundary and equilibrium conditions defined by Eqs. (5) and (3), correspondingly. When disclination dipole arm tends to zero, the relations of Eqs. (68) make it possible to determine the stresses of an edge dislocation perpendicular to the surfaces of a plate; this unique result was first reported in Ref. [44].

\subsection{Wedge disclinations in bodies with spherical geometry}

To solve elasticity boundary-value problems for wedge disclinations described above, we use again the representation of the final elastic field under question as the sum of the singular disclination elastic field (e.g. displacements $u_{k}^{\infty}$ and stresses $\left.\sigma_{i j}^{\infty}\right)$ in infinite medium and an additional field $\left(u_{k}^{\infty}, \sigma_{i j}^{\infty}\right)$ that is responsible for the fulfillment of the boundary conditions at the interfaces and/or external surfaces of the body. In cases of wedge disclinations placed in the bodies with spherical free surfaces we follow the same methodology and find the solution in the form:

$$
\sigma_{i j}^{s}(R, \theta, \varphi)=\sigma_{i j}^{\infty}(R, \theta, \varphi)+\sigma_{i j}^{a}(R, \theta, \varphi),
$$

where we assume symmetrical positioning of wedge disclinations with respect spherical surfaces as it is shown in Figs. 14-16 and use spherical coordinate system $(R, \theta, \varphi)$ with the origin in the point on a wedge disclination line.

In spherical coordinates the stresses $\sigma_{i j}^{\infty}$ given by Eqs. (17) or (18) acquire the other form:

$$
\sigma_{R R}^{\infty}=\frac{G \omega}{2 \pi(1-v)}\left(\frac{1}{2}[1+2 v+(-1+2 v) \cos 2 \theta] \log (R \sin \theta)+\frac{v}{1-2 v}\right),
$$




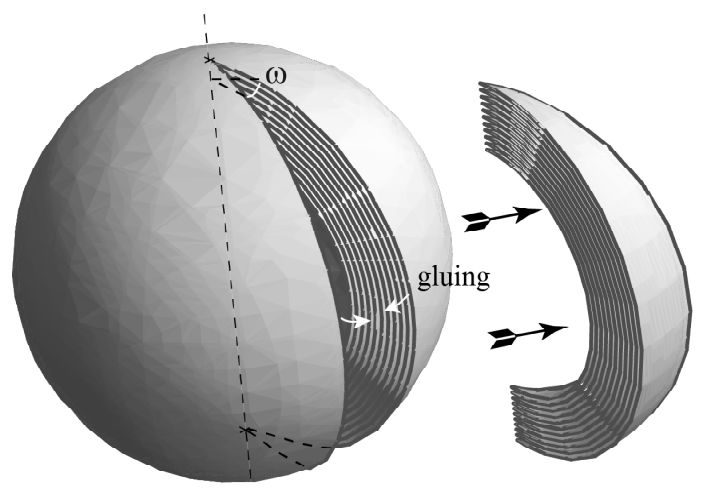

(a)

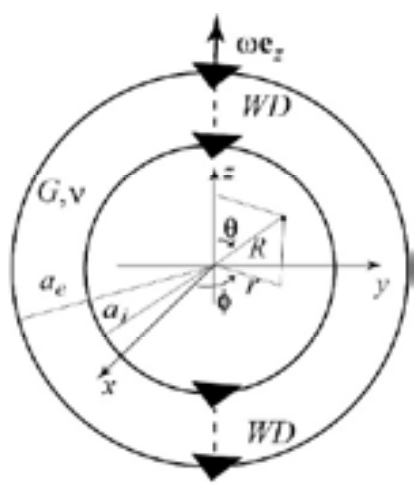

(b)

Fig. 14. Wedge disclination (WD) in an elastic spherical layer (SL). (a) Introduction of a positive WD into the SL; (b) the WD with Frank vector $\omega=\omega \boldsymbol{e}_{z}$ in the SL. The Cartesian $(x, y, z)$ and spherical $(R, \theta, \varphi)$ coordinates are shown. $G$ is the shear modulus and $v$ is the Poisson's ratio of the material of the SL.

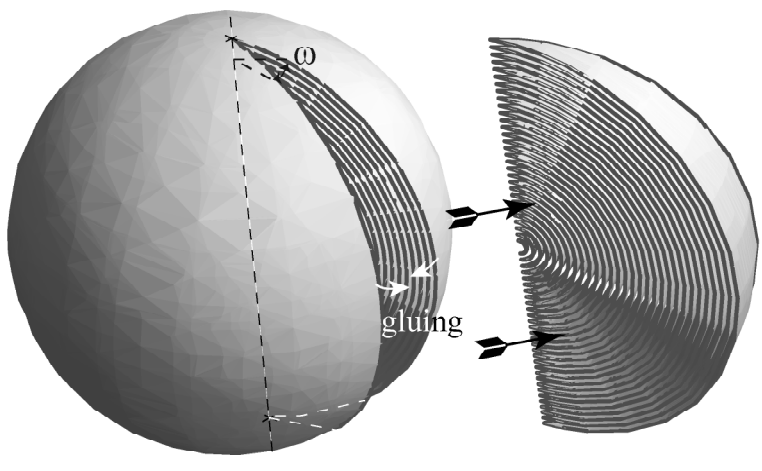

(a)

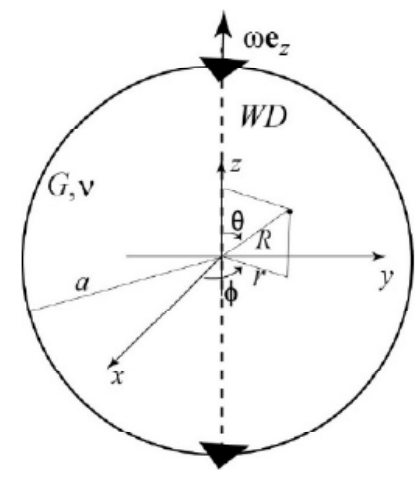

(b)

Fig. 15. Wedge disclination (WD) in a bulk elastic sphere. (a) Introduction of a positive WD into a bulk elastic sphere, (b) a WD with the Frank vector $\omega=\omega \boldsymbol{e}_{z}$ in the bulk sphere of radius $a$.

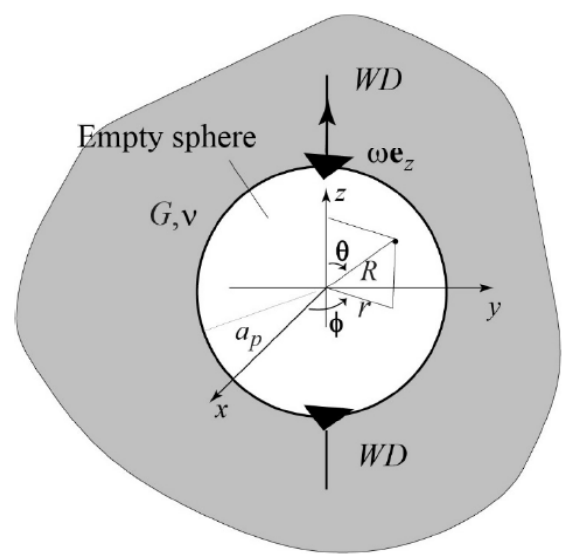

Fig. 16. Wedge disclination (WD) with the Frank vector $\omega=\omega \boldsymbol{e}_{z}$ axially pierces a spherical pore of radius $a_{p}$ in an elastic medium.

$$
\begin{aligned}
& \sigma_{\theta \theta}^{\infty}=\frac{G \omega}{2 \pi(1-v)}\left(\frac{1}{2}[1+2 v+(1-2 v) \cos 2 \theta] \log (R \sin \theta)+\frac{v}{1-2 v}\right), \\
& \sigma_{\varphi \varphi}^{\infty}=\frac{G \omega}{2 \pi(1-v)}\left(\log (R \sin \theta)+\frac{1-v}{1-2 v}\right),
\end{aligned}
$$


$\sigma_{R \theta}^{\infty}=\frac{G \omega}{2 \pi(1-v)}(1-2 v) \cos \theta \sin \theta \log (R \sin \theta)$,

where $0 \leq R<\infty, 0 \leq \theta \leq \pi, 0 \leq \varphi \leq 2 \pi$. Cartesian and spherical coordinates are related by: $x=R \sin \theta \cos \varphi, y=R \sin \theta \cos \varphi$, $z=\cos \theta, R^{2}=x^{2}+y^{2}+z^{2}, \theta=\cos ^{-1}(z / R)$.

For the first time the solution for elastic fields and energy of a wedge disclination in a bulk isotropic sphere was given in Ref. [47]. We however follow the results of Ref. [48] where the solution for a disclination piercing a hollow sphere is first derived and then special cases of a bulk sphere and a spherical pore are considered.

\subsubsection{Disclination piercing a spherical layer}

The procedure of the introduction of a wedge disclination, which goes through sphere diameter, in a spherical layer (SL) with internal and external radii $a_{i}$ and $a_{e}$, respectively, is shown in Fig. 14a. The procedure is very similar to the Volterra process for a wedge disclination in a cylinder that was described in detail in Section 1.

Due to the chosen axial symmetry of the problem, there will be no dependence of disclination elastic fields on the angle $\varphi$ and the stress component $\sigma_{R \varphi}^{S L}$. The elastic field, generated by the wedge disclination in the SL, must satisfy the following four boundary conditions on the SL free surfaces:

$$
\left.\sigma_{R R}^{S L}\right|_{R=a_{i}}=0,\left.\quad \sigma_{R R}^{S L}\right|_{R=a_{e}}=0,\left.\quad \sigma_{R \theta}^{S L}\right|_{R=a_{i}}=0,\left.\quad \sigma_{R \theta}^{S L}\right|_{R=a_{e}}=0 .
$$

The solutions of the elastic problems of this type can be found through the universal equations describing the stress-strain state for a hollow sphere subjected axial symmetric internal and external loading [49]. As it was found in Ref. [48], the stresses of the wedge disclination are written as:

$$
\begin{aligned}
& \sigma_{R R}^{S L}=\sigma_{R R}^{\infty}+2 G \sum_{m=0}^{\infty}\left[\tilde{A}_{m}(2 m+1)\left(4 m^{2}-2 m-2-2 v\right)\left(\frac{R}{a_{e}}\right)^{2 m}+2 \tilde{B}_{m} m(2 m-1)\left(\frac{R}{a_{e}}\right)^{2 m-2}-\right. \\
& \left.2 \tilde{C}_{m} m\left(4 m^{2}+6 m-2 v\right)\left(\frac{R}{a_{i}}\right)^{-(2 m+1)}+\tilde{D}_{m}(2 m+1)(2 m+2)\left(\frac{R}{a_{i}}\right)^{-(2 m+3)}\right] P_{2 m}(\cos \theta), \\
& \sigma_{\theta \theta}^{s L}=\sigma_{\theta \theta}^{\infty}+2 G \sum_{m=0}^{\infty}\left[-\tilde{A}_{m}(2 m+1)\left(4 m^{2}+8 m+2+2 v\right)\left(\frac{R}{a_{e}}\right)^{2 m}-4 \tilde{B}_{m} m^{2}\left(\frac{R}{a_{e}}\right)^{2 m-2}+\right. \\
& \left.2 \tilde{C}_{m} m\left(4 m^{2}-8 m-1+2 v\right)\left(\frac{R}{a_{i}}\right)^{-(2 m+1)}-\tilde{D}_{m}(2 m+1)^{2}\left(\frac{R}{a_{i}}\right)^{-(2 m+3)}\right] P_{2 m}(\cos \theta)- \\
& 2 G \sum_{m=1}^{\infty}\left[\tilde{A}_{m}(2 m+5-4 v)\left(\frac{R}{a_{e}}\right)^{2 m}+\tilde{B}_{m}\left(\frac{R}{a_{2}}\right)^{2 m-2}+\tilde{C}_{m}(-2 m+4-4 v)\left(\frac{R}{a_{i}}\right)^{-(2 m+1)}+\tilde{D}_{m}\left(\frac{R}{a_{i}}\right)^{-(2 m+3)}\right] P_{2 m}^{1}(\cos \theta) \cot \theta, \\
& \sigma_{\varphi \varphi}^{S L}=\sigma_{\varphi \varphi}^{\infty}+2 G \sum_{m=0}^{\infty}\left[\tilde{A}_{m}(2 m+1)(2 m-2-2 v-8 m v)\left(\frac{R}{a_{e}}\right)^{2 m}+2 \tilde{B}_{m} m\left(\frac{R}{a_{e}}\right)^{2 m-2}+\right. \\
& \left.2 \tilde{C}_{m} m(2 m+3-8 m v-2 v)\left(\frac{R}{a_{i}}\right)^{-(2 m+1)}-\tilde{D}_{m}(2 m+1)\left(\frac{R}{a_{i}}\right)^{-(2 m+3)}\right] P_{2 m}(\cos \theta)+ \\
& 2 G \sum_{m=1}^{\infty}\left[\tilde{A}_{m}(2 m+5-4 v)\left(\frac{R}{a_{e}}\right)^{2 m}+\tilde{B}_{m}\left(\frac{R}{a_{e}}\right)^{2 m-2}+\tilde{C}_{m}(-2 m+4-4 v)\left(\frac{R}{a_{i}}\right)^{-(2 m+1)}+\tilde{D}_{m}\left(\frac{R}{a_{i}}\right)^{-(2 m+3)}\right] P_{2 m}^{1}(\cos \theta) \cot \theta, \\
& \sigma_{R \theta}^{S L}=\sigma_{R \theta}^{\infty}+2 G \sum_{m=1}^{\infty}\left[\tilde{A}_{m}\left(4 m^{2}+4 m-1+2 v\right)\left(\frac{R}{a_{e}}\right)^{2 m}+\tilde{B}_{m}(2 m-1)\left(\frac{R}{a_{e}}\right)^{2 m-2}+\right. \\
& \left.\tilde{C}_{m}\left(4 m^{2}-2+2 v\right)\left(\frac{R}{a_{i}}\right)^{-(2 m+1)}-\tilde{D}_{m}(2 m+2)\left(\frac{R}{a_{i}}\right)^{-(2 m+3)}\right] P_{2 m}^{1}(\cos \theta),
\end{aligned}
$$

where $P_{n}(t)$ are the Legendre polynomials and $P_{n}^{1}(t)$ are the associated Legendre polynomials; $t=\cos \theta$, 
$P_{n}^{1}(t)=(-1)\left(1-t^{2}\right)^{-\frac{1}{2}} \frac{d P_{n}(t)}{d t}=\frac{d P_{n}(\cos \theta)}{d \theta}$,

$P_{n}^{1}(\cos \theta)=0$ and the normalized dimensionless terms $\tilde{A}_{m}=A_{m} a_{e}^{2 m}, \tilde{B}_{m}=B_{m} a_{e}^{2 m-2} ; \tilde{C}_{m}=C_{m} a_{i}^{-(2 m+1)} ; \tilde{D}_{m}=D_{m} a_{i}^{-(2 m+3)} ;$ $\tilde{A}_{0}^{n}=A_{0} ; \tilde{D}_{0}=D_{0} a_{i}^{-3}$ are used.

In its turn, the coefficients $A_{m}, B_{m}, C_{m}$, and $D_{m}$ for $m \geq 1$ are determined from the following algebraic system of equations, which holds for any boundary-value problem for spherical layer with boundary conditions of Eqs. (71):

$$
\begin{aligned}
& \sigma_{m}^{i} /(2 G)+A_{m}(2 m+1)\left(4 m^{2}-2 m-2-2 v\right) a_{i}^{2 m}+B_{m} 2 m(2 m-1) a_{i}^{2 m-2}- \\
& C_{m} 2 m\left(4 m^{2}+6 m-2 v\right) a_{i}^{-(2 m+1)}+D_{m}(2 m+1)(2 m+2) a_{i}^{-(2 m+3)}=0, \\
& \sigma_{m}^{e} /(2 G)+A_{m}(2 m+1)\left(4 m^{2}-2 m-2-2 v\right) a_{e}^{2 m}+B_{m} 2 m(2 m-1) a_{e}^{2 m-2}- \\
& \quad C_{m} 2 m\left(4 m^{2}+6 m-2 v\right) a_{e}^{-(2 m+1)}+D_{m}(2 m+1)(2 m+2) a_{e}^{-(2 m+3)}=0, \\
& \tau_{m}^{i} /(2 G)+A_{m}(2 m+1)\left(4 m^{2}+4 m-1+2 v\right) a_{i}^{2 m}+B_{m}(2 m-1) a_{i}^{2 m-2}+ \\
& C_{m}\left(4 m^{2}-2+2 v\right) a_{i}^{-(2 m+1)}-D_{m}(2 m+2) a_{i}^{-(2 m+3)}=0, \\
& \tau_{m}^{e} /(2 G)+A_{m}(2 m+1)\left(4 m^{2}+4 m-1+2 v\right) a_{e}^{2 m}+B_{m}(2 m-1) a_{e}^{2 m-2}+ \\
& \quad C_{m}\left(4 m^{2}-2+2 v\right) a_{e}^{-(2 m+1)}-D_{m}(2 m+2) a_{e}^{-(2 m+3)}=0 .
\end{aligned}
$$

The degenerate case of $m=0$ specifies two remaining and contributing to Eqs. (72) coefficients:

$$
A_{0}=\frac{a_{e}^{3} \sigma_{0}^{e}-a_{i}^{3} \sigma_{0}^{i}}{4 G(1+v)\left(a_{e}^{3}-a_{i}^{3}\right)} \text { and } D_{0}=\frac{a_{e}^{3} a_{i}^{3}\left(\sigma_{0}^{e}-\sigma_{0}^{i}\right)}{4 G\left(a_{e}^{3}-a_{i}^{3}\right)} \text {. }
$$

According to the results presented in Ref. [48], the only quantities that account for the presence of wedge disclination in the layer with the magnitudes of internal $a_{i}$ and external $a_{e}$ radii of the spherical layer are $\sigma_{0}^{i}, \sigma_{0}^{e}, \sigma_{1}^{i}, \sigma_{1}^{e}$, $\tau_{1}^{i}$, and $\tau_{0}^{e}$ :

$$
\begin{aligned}
& \sigma_{0}^{i, e}=\frac{G \omega}{2 \pi(1-v)}\left[\frac{2}{3}(1+v) \ln a_{i, e}+\frac{(2 v-1)}{9}(3 \ln 2-4)+(\ln 2-1)+\frac{v}{1-2 v}\right], \\
& \sigma_{1}^{i, e}=\frac{G \omega}{2 \pi(1-v)} \cdot \frac{\left(16-107 v+30(2 v-1) \ln 2 a_{i, e}\right)}{45}, \\
& \tau_{1}^{i, e}=\frac{G \omega}{2 \pi(1-v)} \cdot \frac{(2 v-1)\left(-31+30 \ln 2 a_{i, e}\right)}{90} .
\end{aligned}
$$

The other coefficients with $m>1$ entering in the system of Eqs. (73), i.e. $\sigma_{m}^{i}, \sigma_{m}^{e}, \tau_{m}^{i}$, and $\tau_{m}^{e}$ include as the parameter only wedge disclination strength $\omega$ :

$$
\begin{aligned}
& \sigma_{m}^{i, e}=\frac{G \omega}{2 \pi(1-v)}\left[\frac{(1-2 v)(2 m-1)(m+1)(4 m+1)}{2(m-1) m(2 m+1)(2 m+3)}-\frac{(4 m+1)}{2 m(2 m+1)}\right], m=2,3,4 \ldots \\
& \tau_{m}^{i, e}=\frac{G \omega}{2 \pi(1-v)} \cdot \frac{(1-2 v)(4 m+1)}{2(m-1) m(2 m+1)(2 m+3)}, m=2,3,4 \ldots
\end{aligned}
$$

The set of the listed Eqs. (72) to (75) allows to investigate the distribution of mechanical stresses in a spherical layer with a wedge disclination that was demonstrated on the example of stress maps in Ref. [48]. 


\subsubsection{Disclination in a bulk sphere}

In the case of a wedge disclination in a bulk sphere (see Fig. 15) the solution of elasticity boundary-value problem can be found utilizing formulas from previous Section and assuming $a_{i}=0$ and $a_{e}=a$ with $a$ being sphere radius. For a sake of completeness, we write here the stresses $\sigma_{i j}^{B S}$ according to the formulas given in Ref. [48]:

$$
\begin{aligned}
& \sigma_{R R}^{B S}=\sigma_{R R}^{\infty}+2 G \sum_{m=0}^{\infty}\left[\tilde{A}_{m}(2 m+1)\left(4 m^{2}-2 m-2-2 v\right)\left(\frac{R}{a}\right)^{2 m}+2 \tilde{B}_{m} m(2 m-1)\left(\frac{R}{a}\right)^{2 m-2}\right] P_{2 m}(\cos \theta), \\
& \sigma_{\theta \theta}^{B S}=\sigma_{\theta \theta}^{\infty}+2 G \sum_{m=0}^{\infty}\left[-\tilde{A}_{m}(2 m+1)\left(4 m^{2}+8 m+2+2 v\right)\left(\frac{R}{a}\right)^{2 m}-4 \tilde{B}_{m} m^{2}\left(\frac{R}{a}\right)^{2 m-2}\right] P_{2 m}(\cos \theta)- \\
& 2 G \sum_{m=1}^{\infty}\left[\tilde{A}_{m}(2 m+5-4 v)\left(\frac{R}{a}\right)^{2 m}+\tilde{B}_{m}\left(\frac{R}{a}\right)^{2 m-2}\right] P_{2 m}^{1}(\cos \theta) \cot \theta, \\
& \sigma_{\varphi \varphi}^{B S}=\sigma_{\varphi \varphi}^{\infty}+2 G \sum_{m=0}^{\infty}\left[\tilde{A}_{m}(2 m+1)(2 m-2-2 v-8 m v)\left(\frac{R}{a}\right)^{2 m}+2 \tilde{B}_{m} m\left(\frac{R}{a}\right)^{2 m-2}\right] P_{2 m}(\cos \theta)+ \\
& 2 G \sum_{m=1}^{\infty}\left[\tilde{A}_{m}(2 m+5-4 v)\left(\frac{R}{a}\right)^{2 m}+\tilde{B}_{m}\left(\frac{R}{a}\right)^{2 m-2}\right] P_{2 m}^{1}(\cos \theta) \cot \theta, \\
& \sigma_{R \theta}^{B S}=\sigma_{R \theta}^{\infty}+2 G \sum_{m=1}^{\infty}\left[\tilde{A}_{m}\left(4 m^{2}+4 m-1+2 v\right)\left(\frac{R}{a}\right)^{2 m}+\tilde{B}_{m}(2 m-1)\left(\frac{R}{a}\right)^{2 m-2}\right] P_{2 m}^{1}(\cos \theta),
\end{aligned}
$$

where

$$
\begin{aligned}
& \tilde{A}_{0}=\frac{\sigma_{0}}{4 G(1+v)}, \tilde{A}_{m}=\frac{\left(\sigma_{m}-2 m \tau_{m}\right)}{4 G[1+v+2 m(1+2 m+2 v)]} \\
& \text { and } \tilde{B}_{m}=-\frac{(2 v-1+4 m(1+m)) \sigma_{m}+2\left(1+v-4 m^{3}+m(3+2 v)\right) \tau_{m}}{4 G(2 m-1)[1+v+2 m(1+2 m+2 v)]}
\end{aligned}
$$

( $m \geq 1$ ) with $\sigma_{m}$ and $\sigma_{m}$ defined by Eqs. (74) and (75) by substituting $a_{e}$ with $a$.

We note that elasticity solution for a wedge in an isotropic bulk sphere were given in the other form in Ref. [47] where, in addition, the expression for elastic energy of disclinated sphere was provided:

$$
E^{B S}=\frac{G \omega^{2} a^{3}}{2 \pi(1-v)}\left(\frac{1}{6}-\sum_{m=1}^{\infty} \frac{(4 m+3)\left(8 v^{2} m^{2}+2 m\left(5 v^{2}+3 v-1\right)+(1+v)(1+2 v)\right)}{2 m(m+1)(2 m+1)(2 m+3)^{2}\left(4 m^{2}+2 m(1+2 v)+1+v\right)}\right) .
$$

\subsubsection{Disclination running through spherical cavity}

The solution for stresses $\sigma_{i j}^{p}$ of a wedge disclination intersecting a spherical cavity (pore) of radius $a_{p}$ (see Fig. 16) can be extracted from general formulas of Eqs. (72) for stresses of a disclination in a spherical layer by setting $a_{i}=a_{p}$ and $a_{e}=\infty$.

The other straight-forward way to get stresses $\sigma_{i j}^{p}$ that satisfy the boundary conditions on the pore free surface was given in Ref. [45] where the result was written as:

$$
\begin{aligned}
& \sigma_{R R}^{P}=\sigma_{R R}^{\infty}+2 G \sum_{m=0}^{\infty}\left[2 \tilde{C}_{m} m\left(4 m^{2}+6 m-2 v\right)\left(\frac{R}{a_{p}}\right)^{-(2 m+1)}+\tilde{D}_{m}(2 m+1)(2 m+2)\left(\frac{R}{a_{p}}\right)^{-(2 m+3)}\right] P_{2 m}(\cos \theta), \\
& \sigma_{\theta \theta}^{P}=\sigma_{\theta \theta}^{\infty}+2 G \sum_{m=0}^{\infty}\left[2 \tilde{C}_{m} m\left(4 m^{2}-8 m-1+2 v\right)\left(\frac{R}{a_{p}}\right)^{-(2 m+1)}-\tilde{D}_{m}(2 m+1)^{2}\left(\frac{R}{a_{p}}\right)^{-(2 m+3)}\right] P_{2 m}(\cos \theta)- \\
& 2 G \sum_{m=1}^{\infty}\left[\tilde{C}_{m}(-2 m+4-4 v)\left(\frac{R}{a_{p}}\right)^{-(2 m+1)}+\tilde{D}_{m}\left(\frac{R}{a_{p}}\right)^{-(2 m+3)}\right] P_{2 m}^{1}(\cos \theta) \cot \theta,
\end{aligned}
$$




$$
\begin{aligned}
& \sigma_{\varphi \varphi}^{P}=\sigma_{\varphi \varphi}^{\infty}+2 G \sum_{m=0}^{\infty}\left[2 \tilde{C}_{m} m(2 m+3-8 m v-2 v)\left(\frac{R}{a_{p}}\right)^{-(2 m+1)}-\tilde{D}_{m}(2 m+1)\left(\frac{R}{a_{p}}\right)^{-(2 m+3)}\right] P_{2 m}(\cos \theta)+ \\
& 2 G \sum_{m=1}^{\infty}\left[\tilde{C}_{m}(-2 m+4-4 v)\left(\frac{R}{a_{p}}\right)^{-(2 m+1)}+\tilde{D}_{m}\left(\frac{R}{a_{p}}\right)^{-(2 m+3)}\right] P_{2 m}^{1}(\cos \theta) \cot \theta, \\
& \sigma_{R \theta}^{P}=\sigma_{R \theta}^{\infty}+2 G \sum_{m=1}^{\infty}\left[\tilde{C}_{m}\left(4 m^{2}-2+2 v\right)\left(\frac{R}{a_{p}}\right)^{-(2 m+1)}-\tilde{D}_{m}(2 m+2)\left(\frac{R}{a_{p}}\right)^{-(2 m+3)}\right] P_{2 m}^{1}(\cos \theta),
\end{aligned}
$$

Here

$$
\tilde{D}_{0}=-\frac{\sigma_{0}^{(p)}}{4 G}, \quad \tilde{C}_{m}=\frac{\sigma_{m}^{(p)}+\tau_{m}^{(p)}+2 m \tau_{m}^{(p)}}{4 G\left(1-v+2 m\left(1-4 m-4 m^{2}\right)\right)} \text { and } \tilde{D}_{m}=\frac{\left(1-v-2 m^{2}\right) \sigma_{m}^{(p)}+2 m\left(3 m+2 m^{2}-v\right) \tau_{m}^{(p)}}{4 G(1+m)\left(1-v+2 m\left(1-4 m-4 m^{2}\right)\right)}
$$

for $(m \geq 1)$ with $\sigma_{m}^{(p)}$ and $\tau_{m}^{(p)}$ defined by Eqs. (74) and (75) by substituting $a_{i}$ with $a_{p}$.

One may note that the formulas written above have a restricted physical meaning because there is no screening for disclination elastic filed at large distances from the pore and logarithmic divergence of disclination stresses remains.

\subsection{Conic disclinations in an elastic sphere}

Considering a bulk sphere, we can imagine another defect - a conical disclination, which formation is described by a procedure being very similar to the Volterra process for a wedge disclination in a cylinder. Localized conical disclination is introduced in a sphere by cutting and removing a cone of solid angle $\chi$ with the subsequent contraction and gluing of the surface of the conical dimple along the radius of the sphere as shown in Fig. 17a. Alternatively, an additional solid conus can be inserted in a solid sphere. In the first case we have a positive localized conical disclination, and in the second - a negative one. Plastic strain (eigenstrain) for a positive conical disclination has the following form:

$\varepsilon_{\theta \theta}^{*}=\varepsilon_{\varphi \varphi}^{*}=-\chi \delta\left(\cos \theta-\cos \theta_{0}\right) \delta\left(\varphi-\varphi_{0}\right) H(a-R)$,

where $\delta(\xi)$ is the Dirac delta-function, $H(z)$ is the Heaviside step function, $(R, \theta, \varphi)$ is the spherical coordinate system, $\theta_{0}$ and $\varphi_{0}$ are the angle coordinates of the gluing radius, $a$ is the radius of the sphere.

Elastic fields for such conical disclination can be, in principle, found exploring the general approach for axisymmetrically loaded sphere [49]. However, till now the problem for localized conical defect has no published solution. It should be noted that this solution in case it will be found, will demonstrate singularity of stress and strain fields along the sphere radius but not only in the sphere center. This means that, following our classification, localized conical disclination is a linear defect.

As an alternative to the localized conical disclination, the authors of Ref. [50] have described another defect that can be defined by considering a sphere with a deficit in solid angle $\chi_{M Y}$, which is spread uniformly through the entire sphere, see Fig. 17b. Such a defect can be visualized as a set of infinite number of infinitesimally thin empty radial cones with solid angle $d \mathrm{c}$ each. Then each of the cones is subjected to the procedure that was described in the beginning of this section providing the following plastic strain distributed uniformly through the whole volume of the sphere:

$\varepsilon_{\theta \theta}^{*}=\varepsilon_{\varphi \varphi}^{*}=-\chi_{M Y} H(a-R)$

We name such a defect as a stereo disclination or Marks-Yoffe (MY) disclination [28,51-53]. The stresses for MY disclination are derived from Eq. (81), they have to satisfy boundary conditions on the free surface of a sphere; for the first time they were given in Ref. [50]:

$$
\begin{aligned}
& \sigma_{R R}^{M Y}=\frac{4 G \chi_{M Y}}{3} \frac{1+v}{1-v} \log \frac{R}{a}, \\
& \sigma_{\theta \theta}^{M Y}=\sigma_{\varphi \varphi}^{M Y}=\frac{4 G \chi_{M Y}}{3} \frac{1+v}{1-v}\left(\log \frac{R}{a}+\frac{1}{2}\right) .
\end{aligned}
$$




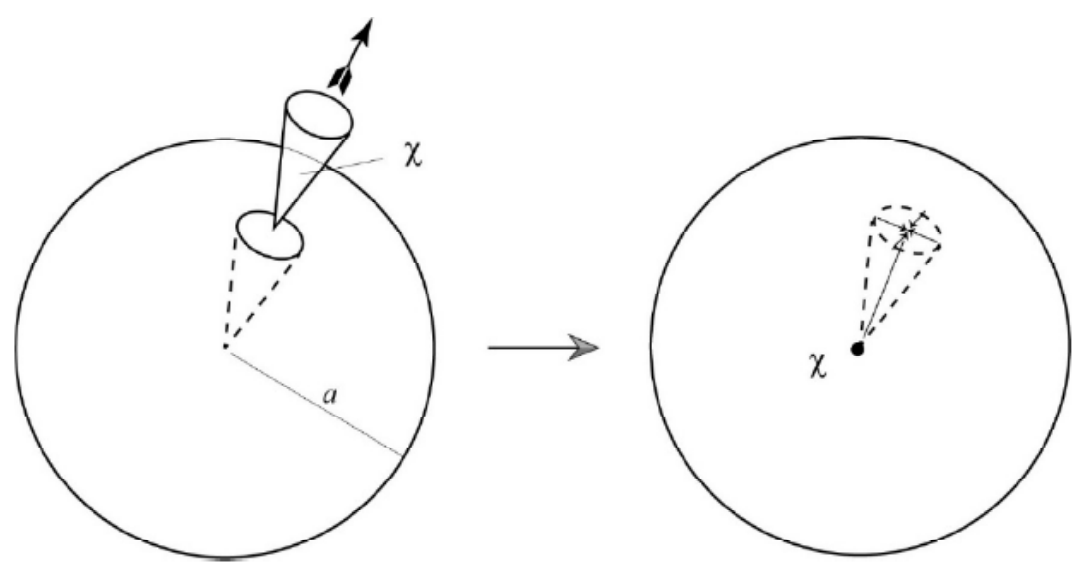

(a)

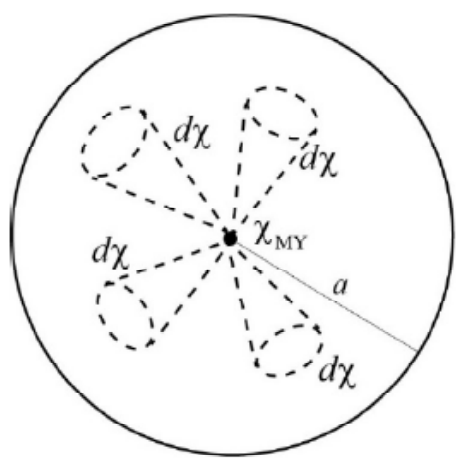

(a)

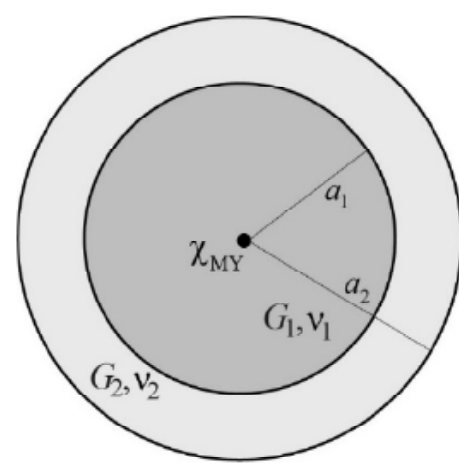

(b)

Fig. 17. Conical disclinations in a bulk sphere. (a) Procedure of formation of singular conical disclination (CD) of strength $\chi$ in an elastic sphere, (b) distributed conical disclinations - stereo or Marks-Yoffe (MY) disclination of strength $\chi_{M Y}$, (c) MY disclination in a two-phase sphere.

Then, the stored elastic energy $E^{M Y}$ associated with the stress field of MY disclination is:

$E^{M Y}=\frac{8 \pi G}{27} \frac{1+v}{1-v} \chi_{M Y}^{2} a^{3}$

The elastic properties of MY disclination in a two-phase elastic sphere (see Fig. 17c) were analyzed in Ref. [28], where the following formulas for stresses and elastic energy of this defect were found:

(i) for $R \leq a_{i}$ :

$$
\begin{aligned}
& \sigma_{R R}^{M Y 1}=G_{1} \chi_{M Y}\left[\frac{4}{3} \frac{1+v_{1}}{1-v_{1}} \log \frac{R}{a_{1}}+\right. \\
& \left.\frac{G_{2}\left(1+v_{1}\right)\left(1+v_{2}\right) a_{2}^{3}}{G_{2}\left(1-2 v_{1}\right)\left(1+v_{2}\right)\left(a_{2}^{3}-a_{1}^{3}\right)+G_{1}\left(1-2 v_{2}\right)\left(1+v_{1}\right) a_{1}^{3}+G_{1}\left(1+v_{1}\right)\left(1+v_{2}\right) a_{2}^{3}} \log \frac{a_{1}}{a_{2}}\right], \\
& \sigma_{\theta \theta}^{M Y 1}=\sigma_{\varphi \varphi}^{M Y 1}=G_{1} \chi_{M Y}\left[\frac{4}{3} \frac{1+v_{1}}{1-v_{1}}\left(\log \frac{R}{a_{1}}+\frac{1}{2}\right)+\right. \\
& \left.\frac{G_{2}\left(1+v_{1}\right)\left(1+v_{2}\right) a_{2}^{3}}{G_{2}\left(1-2 v_{1}\right)\left(1+v_{2}\right)\left(a_{2}^{3}-a_{1}^{3}\right)+G_{1}\left(1-2 v_{2}\right)\left(1+v_{1}\right) a_{1}^{3}+G_{1}\left(1+v_{1}\right)\left(1+v_{2}\right) a_{2}^{3}} \log \frac{a_{1}}{a_{2}}\right],
\end{aligned}
$$


(ii) for $a_{1} \leq R \leq a_{2}$ :

$$
\begin{aligned}
& \sigma_{R R}^{M Y 2}=G_{2} \chi_{M Y}\left[\frac{4}{3} \frac{1+v_{2}}{1-v_{2}}\left(\log \frac{R}{a_{2}}+\frac{R^{3}-a_{2}^{3}}{a_{2}^{3}-a_{1}^{3}} \frac{a_{1}^{3}}{R^{3}} \log \frac{R}{a_{1}}\right)+\right. \\
& \left.\frac{G_{1}\left(1+v_{1}\right)\left(1+v_{2}\right) a_{1}^{3}}{G_{2}\left(1-2 v_{1}\right)\left(1+v_{2}\right)\left(a_{2}^{3}-a_{1}^{3}\right)+G_{1}\left(1-2 v_{2}\right)\left(1+v_{1}\right) a_{1}^{3}+G_{1}\left(1+v_{1}\right)\left(1+v_{2}\right) a_{2}^{3}} \frac{a_{2}^{3}\left(a_{2}^{3}-R^{3}\right)}{R^{3}\left(a_{2}^{3}-a_{1}^{3}\right)} \log \frac{a_{1}}{a_{2}}\right], \\
& \sigma_{\theta \theta}^{M Y 2}=\sigma_{\varphi \varphi}^{M Y 2}=G_{2} \chi_{M Y}\left[\frac{4}{3} \frac{1+v_{2}}{1-v_{2}}\left(\log \frac{R}{a_{2}}+\frac{R^{3}-a_{2}^{3}}{a_{2}^{3}-a_{1}^{3}} \frac{a_{1}^{3}}{R^{3}} \log \frac{R}{a_{1}}+\frac{1}{2}\right)-\right. \\
& \left.\frac{G_{1}\left(1+v_{1}\right)\left(1+v_{2}\right) a_{1}^{3}}{G_{2}\left(1-2 v_{1}\right)\left(1+v_{2}\right)\left(a_{2}^{3}-a_{1}^{3}\right)+G_{1}\left(1-2 v_{2}\right)\left(1+v_{1}\right) a_{1}^{3}+G_{1}\left(1+v_{1}\right)\left(1+v_{2}\right) a_{2}^{3}} \frac{a_{2}^{3}\left(a_{2}^{3}+2 R^{3}\right)}{2 R^{3}\left(a_{2}^{3}-a_{1}^{3}\right)} \log \frac{a_{1}}{a_{2}}\right], \\
& E^{M Y 2}=\frac{8 \pi G_{2}}{27} \chi_{M Y}^{2} a_{2}^{3}\left[\frac{1+v_{2}}{1-v_{2}}\left(\frac{a_{2}^{3}-a_{1}^{3}}{a_{2}^{3}}\right)+\frac{1+v_{1}}{1-v_{1}} \frac{G_{1}}{G_{2}} \frac{a_{1}^{3}}{a_{2}^{3}}+9 \frac{a_{1}^{3}}{a_{2}^{3}-a_{1}^{3}} \times\right. \\
& \left.\left(\frac{3 G_{1}\left(1+v_{1}\right)\left(1+v_{2}\right) a_{2}^{3}}{G_{2}\left(1-2 v_{1}\right)\left(1+v_{2}\right)\left(a_{2}^{3}-a_{1}^{3}\right)+G_{1}\left(1-2 v_{2}\right)\left(1+v_{1}\right) a_{1}^{3}+G_{1}\left(1+v_{1}\right)\left(1+v_{2}\right) a_{2}^{3}}-\frac{1+v_{2}}{1-v_{2}}\right) \log ^{2} \frac{a_{1}}{a_{2}}\right],
\end{aligned}
$$

where $a_{1}$ is the radius of the spherical phase 1 and $a_{2}$ is the external radius of the spherical layer of phase 2 , while $G_{1}$, $v_{1}$ and $G_{2}, v_{2}$ are used to denote elastic moduli for phases 1 and 2, respectively.

Obviously, rather cumbersome expressions (84) and (86) transform to simpler formulas (82) and (83) making $a_{2}=a_{1}=a$ or allowing $G_{2}=0$.

\section{APPLICATIONS}

In this section, we present the information in brief on the use of disclination models in modern Materials Science and Solid State Physics to explain or even predict various phenomena in 3D and 2D crystalline and amorphous solids. The emphasis is made on the models exploring the properties of straight wedge disclinations. We only designate the areas of application of the disclination approach providing the relevant references. Detailed information on the subject can be found in the referred articles, but also in a number of books [4,5,54-57] and reviews [6,9,10,58-62] published during last four decades.

\subsection{Disclinations and rotational plasticity}

For the first time, the idea to use wedge disclinations in explanation of crystal plasticity phenomena was given in Ref. [63] in application to deformation twinning. After that, twins were modelled with help of disclination dipoles and quadrupoles [64-66]. Important feature of all the referred works is an attempt to relate rotation (inclination) of crystallographic planes peculiar to twinning with the rotational defects - disclinations.

In general, rotational effects were observed in crystal plasticity starting the first application (in the first quarter of the last century) of X-rays to the analysis of crystal structure evolution in the course of plastic deformation; for historical survey the readers can be addressed to the Section 3 of Ref. [4]. In the 1970-80s this phenomenon was analyzed in detail for metals subjected to large plastic deformations, where it got the name - fragmentation [55]. It was argued that partial wedge disclinations play a crucial role in fragmentation initiation [55,56,70-75].

An important result was the explanation of the development of so-called reorientation bands in crystals based on the model of disclination dipole motion [5,76,77]. This model with modifications was successfully used in the analysis of kink band nucleation and propagation in materials [54,58,78,79] including those with layer or fiber structure [80-83]. Kinking and twining phenomena have a lot in common; they both are realized when the operation of dislocation slip systems in the direction of the acting shear stress is prohibited or hindered. The difference is in the fact that the misorientation of crystal parts resulting from twinning is strictly defined by crystallographic constrains whereas for kink (and reorientation) bands misorientations dependend on the conditions of mechanical loading.

The models exploring properties of wedge disclinations were applied in explanation deformation mechanisms in polycrystals with nanoscale grains (also known as nanocrystalline materials or nanocrystals) and unltrafine-grained 
materials [56,59,84-90] where disclination related workhardening becomes an important feature [89-94]. Finally, of great importance are the disclination description of deformation mechanisms in rock materials [95] and the involvement of disclinations in the explanation of superplastic behavior of ceramics [96].

\subsection{Disclinations and grain boundaries}

We start this section with the citate "One of the main reasons is that the disclination is a rotational defect while the dislocation is a translational defect. A grain boundary, being a rotational defect, should be described more simply by disclinations" taken from Ref. [97], where it was proposed to model high-angle tilt grain boundaries (GBs) as chains of alternating sign wedge disclinations and to calculate in such a framework the energy of GBs in dependence of the misorientation (tilt) angle. Note that the same article [97] did present the compact formulas for elastic fields and energies of interacting wedge disclinations.

Later on, the disclination model was modified in Ref. [98] to find the energies of tilt GBs between so-called cusp misorientations, for which GBs possess local minima of energy because of their preferable atomic structure. The next step in applying disclination approach to the analysis of GB properties was accounting for structural units - the elements of GBs of finite length [99-102]. The disclination-structural units (DSU) model was then combined with atomistic simulations to calculate GB energies for various materials starting from the potential of interatomic interactions [103-106]. Based on DSU model the authors of Ref. [107] developed a unique approach for predicting the properties (elastic fields and energy) of so-called nonequilibrium grain boundaries. Nonequilibrium boundaries demonstrate excess energy for the prescribed average misorientation depending on the character of disclination dipole distribution in the plane of a GB. In Refs. [108-110] the idea on disclination mediated nonequilibrium state of GBs was extended to so-called quasi-periodic GBs and GBs of finite extent.

The other important elements of the defect structure peculiar to conventional crystals but also nanocrystals - triple junctions of GBs, in many cases demonstrate wedge disclination counterpart. It was clearly demonstrated in Refs. [55,57,72,111,112] that nonuniformity and anisotropy of plastic deformation in neighboring grains lead to the formation of disclinations in GB junctions. The strength of such defects that we designate as Rybin's disclinations depends on the crystallographic orientation and intensity of dislocation slip in each of grains adjusting to the GB junction.

Junction disclinations were recognized to be responsible for the initiation of fragmentation in polycrystals
[55-58,70-75,111-113] and for grain refinement for achieving nanoscale grain size in the course of severe plastic deformation [59,114-117]. In the previous Section we have already mentioned the role of disclinations in controlling mechanical properties of nanocrystalline materials [59,84-90]; here we can only note that disclinations there are at most junction located defects. The evolution of junction wedge disclination configurations in nanocrystyalline materials were studied in Refs. $[118,119]$. Finally, the techniques for identification of junction disclinations from the transmission electron microscopy and X-ray diffraction data were proposed in Refs. [120] and [121], respectively.

\subsection{Crack nucleation at disclinations. Diffusion in an elastic field of disclinations}

Various configuration of wedge disclinations serve as strong sources of tensile stresses either in the vicinity of their cores or even at some distances depending on disclination sign and the character on boundary conditions. Therefore, disclinations can be considered as potential places for fracture initiation in solids.

First disclination models for microcrack nucleation in the elastic field of wedge disclinations were proposed in Refs. [122,123], where the fracture mechanics analysis was performed accounting for internal stresses being peculiar to various disclination dipole and quadrupole configurations. In the following studies external loading was included in consideration as it usually done when analyzing critical conditions for crack opening in the vicinity of stress concentrator. Crack initiation in loaded [124] and unloaded [126-128] disclinated cylinder (containing a negative wedge disclination) was considered using analytical models [124-126] and molecular dynamics (MD) simulation [127,128]. The crack behavior at various disclination dipole configurations in loaded infinite elastic media was studied in Refs. [129131]. Other models for crack formation in the presence of disclinations included various aspects of disclination screening [132-134] and blocked deformation twins $[135,136]$.

Wedge disclinations also contribute to the processes of diffusion in crystalline solids. For the first time the problem of stationary diffusion of point defects in the elastic field of a single wedge disclination was posted and solved in Ref. [137]. After that diffusion related effects were analyzed for disclinations with various physical applications [138-141]. As a result of vacancy diffusion, a cavity can be formed in the core region of positive disclination in a cylinder [142] or Marks-Yoffe disclination in a sphere $[142,143]$. 


\subsection{Disclinations in pentagonal rods and icosahedral particles}

Two last mentioned in the previous Section references already deal with micro-objects demonstrating unusual for crystalline solids five-fold symmetry. It was argued as early as in the beginning of 1970s [144,145] that such objects are formed due to multiple twinning in FCC crystal lattice and the presence of wedge disclinations. Following these ideas, it was proposed to model pentagonal micro- and nanorods as a cylinder with a single positive wedge disclination $[28,51,144]$ and icosahedral micro- and nanoparticles - as a bulk sphere either with six wedge disclinations [51] or with Marks-Yoffe stereo disclination [50,51].

Knowledge on elastic fields and energies of disclinated cylinder and sphere allowed to investigate and to predict many structural features being peculiar to pentagonal micro- and nano-objects, for a review see Ref. [51]. The main observation that can be well explained on the basis of disclination approach is the manifestation of various relaxation processes in the structure of pentagonal particles and rods emerging with the increase of their diameter [146-148]. These relaxation processes were then treated in full details for the formation in pentagonal objects additional dislocations [149-151] disclinations [152,153], cracks [154], or lattice-mismatched surface layers $[52,53,155,156]$.

\subsection{Disclinations and amorphous state}

Wedge disclinations are recognized to be essential elements of internal structure of glasses and amorphous solids [157-162]. They are responsible for elimination of long-range translational periodicity peculiar to conventional crystals and constitute themselves in the appearance of odd-member rings of atomic bonds.

Elastic properties of disclinations were explored in calculating the difference of internal energy between amorphous and crystalline state [26], in the analysis of flow stress of metallic glasses [163], and in the analysis of the structure of crystal-glass interfaces [164].

\subsection{Domains in ferroelastic films}

Film and layers of various crystalline materials may exhibit phase transformation under mechanically constrained conditions when they are deposited on the substrate [165]. If the phase transformation is accompanied with a change of the symmetry of elementary crystal cell, elastic domain structures develop in the film interior, e.g. see Refs. [166,167].

To analyze important features of such domain structures including the dependence of the domain structure period on the film thickness a number of disclination based models has been developed [168-177]. In these models, the effects of mutual disclination screening in multipole configurations as well as disclination interactions with film free surface were taken into account.

\subsection{Wedge disclinations in graphene}

In graphene $-2 \mathrm{D}$ material with a pristine crystal structure made of carbon atom hexagons, wedge disclinations are natural defects that change local symmetry of atomic rings from six-fold to five- or seven-fold and even to four- or eight-fold [24,60].

Disclinations were used in analyzing the properties of carbon allotropes: fullerenes [178], nanotubes [179], nanocones [180], and graphene [60] exploring both continuum [181-183] and atomistic [184-187] approaches. Useful results were delivered when modeling with the help of disclinations the properties of grain boundaries (GBs) and interfaces in graphene that included the description of non-equilibrium GBs with excess energy [182] and zero-misorientation interfaces [187] as well as when analyzing crack initiation at disclinated GBs [188]. In last decade, pseudo-graphenes - planar graphene allotropes with periodically distributed disclinations, attracted special attention of researchers $[189,60,61]$. A remarkable fact on pseudo-graphenes is that they fist predicted only theoretically were recently experimentally synthesized [190].

\section{SUMMARY AND CONCLUDING REMARKS}

In this review, we have reported on the elastic properties, e.g. elastic fields and elastic energies, of straightlinear wedge disclinations in the cases of their interaction with a free surface of various geometries: planar, cylindrical, and spherical ones. As a starting point the solution for elastic fields generated by an isolated wedge disclination in an infinite elastically isotropic continuum have been used. This solution has no direct physical meaning but can be used for construction of the fields of screened disclination configurations: wedge disclination dipoles and quadropoles and dislinations in the bodies of finite extent.

The following boundary-value problems of plane elasticity for single wedge disclination and their ensembles have been considered: disclinations parallel to a free surface of a half-space, disclination in the plate of a finite thickness, and disclinations in uniform and twophase cylinders. In addition, the interaction of wedge disclinations with interfaces have been addressed.

The solutions of three-dimensional elasticity problem have been given for wedge disclinations, whose lines are perpendicular to a free surface of a half-space, or to the surfaces of the plate of a finite thickness. For 
bodies with spherical boundaries the cases of bulk and hollow spheres, as well as disclination piercing a spherical pore have been analyzed. For uniform and two-phase bulk sphere the elastic properties of Marks-Yoffe stereo disclination have been also delivered.

Finally, possible applications of the elasticity solutions for wedge disclinations have been discussed. It has been demonstrated that the disclination properties become a controlling factor when considering rotational plasticity in solids, grain boundaries and their junctions in conventional polycrystals and nanostructured materials, crack nucleation and initiation of ductile fracture, pentagonal rods and icosahedral micro- and nanoparticles, amorphous solids and glasses, domains, and twins in ferroelastic films adjusted to a bulk substrate, and defects in graphene.

Except isotropic linear elasticity more involved cases for materials properties were probed when studying the properties of wedge disclinations. Elastic anisotropy was taken into account in Refs. [191-197] whereas nonlinear elasticity accounting both for geometrical and physical nonlinear effects was used in Refs. [198-202] in finding disclination elastic strains and stresses. Other variants of elasticity models applied to wedge disclinations included exploration of gradient elasticity [203,204], consideration of surface/interface stresses [205,206], or the analysis in the framework of micropolar elasticity [207].

In conclusion, it is worth to note the relation of wedge disclinations to singularities of physical fields of various nature. In this connection, gauge theory [208] and topological approach [209] can be used for the analysis of the media with disclinations. Moreover, the analogies with general physical theories of Macro- and Microworld can be established [210].

\section{ACKNOWLEDGEMENTS}

The work under this review was performed under the financial support from Russian Science Foundation grant \# 19-19-00617.

\section{REFERENCES}

[1] F.R.N. Nabarro, Theory of Crystal Dislocations, Clarendon Press, Oxford, 1967.

[2] N.D. Mermin, Topological theory of defects in ordered media, Rev. Modern Phys., 1979, vol. 51, no. 3, pp. 591-648. https://doi.org/10.1103/ $\underline{\text { RevModPhys.51.591 }}$

[3] M. Kleman, Points, Lines and Walls, Wiley, New York, 1983.

[4] A.E. Romanov and V.I. Vladimirov, Disclinations in Crystalline Solids, In: Dislocations in Solids, vol. 9, ed. by F.R.N. Nabarro, North-Holland, Amsterdam, 1992, p. 191-402.
[5] D.R. Nelson, Defects and Geometry in Condensed Matter Physics, Cambridge University Press, Cambridge, 2002.

[6] A.E. Romanov and A.L. Kolesnikova, Application of disclination concept to solid structures, Prog. Mater. Sci., 2009, vol. 4, no. 6, pp. 740-769. https://doi.org/10.1016/j.pmatsci.2009.03.002

[7] F.C. Frank, On the theory of liquid crystals, Disc. Farad. Soc., 1958, vol. 25, pp. 19-28. https://doi.org/10.1039/df9582500019

[8] V. Volterra, Sur l'équilibre des corps élastiques multiplement connexes, Annales scientifiques de l'École Normale Supérieure Paris, 1907, vol. 24, pp. 401-518.

[9] A.E. Romanov and V.I. Vladimirov, Disclinations in Solids, Phys. Stat. Solidi (a), 1983, vol. 78, no. 1, pp. 11-34. https://doi.org/10.1002/pssa.2210780102

[10] A.E. Romanov, Mechanics and physics of disclinations in solids, Eur. J. Mech. A / Solids, 2003, vol. 22, no. 5, pp. 727-741. https://doi.org/10.1016/S0997-7538(03)00089-5

[11] A.I. Lurie, Theory of elasticity, Nauka, Moscow, 1970, in Russian.

[12] T. Mura, Micromechanics of Defects in Solids, Martinus Nijhoff, Boston, 1987.

[13] C. Somigliana, Sulla toria delle distosioni elastiche, Rend. Reale Accad. Lincei, 1915, vol. 24, no. 1, pp. 655-666.

[14] K.D. Hjelmstad, Fundamentals of Structural Mechanics, Springer, Boston, 2005.

[15] A.L. Kolesnikova and A.E. Romanov, Circular dislocation-disclination loops and their application to boundary problem solution in the theory of defects, Preprint no. 1019, Ioffe Physical-Technical Institute, Leningrad, 1986, in Russian.

[16] R. de Wit, Theory of disclinations: III Continuous and discrete disclinations in isotropic elasticity, J. Res. Nat. Bur. Stand., 1973, vol. 77A, no. 3, pp. 359-368. https://doi.org/10.6028/jres.077A.024

[17] R. de Wit, Linear theory of static disclinations, In: Fundamental aspects of dislocations, vol. 317(I), ed. by J.A. Simmons, R. de Wit and R. Bullough, Nat. Bur. Stand. (US), Spec. Publ. 1970, p. 651-673.

[18] T. Mura, The continuum theory of dislocations, In: Advances in Materials Research, vol. 3, ed. by H. Herman (Interscience Publ., New York, 1968), p. 1-108.

[19] T. Mura, Semi-microscopic plastic distortion and disclinations, Arch. Mech., 1972, vol. 24, no. 3, pp. 449-456.

[20] R. de Wit, Theory of disclinations: IV Straight disclinations, J. Res. Nat. Bur. Stand., 1973. vol. 
77A, no. 5, pp. 607-658. https://doi.org/10.6028/ jres.077A.036

[21] N.A. Pertsev, A.E. Romanov and V.I. Vladimirov, Rectangular disclination loops. I. A universal technique, Phil. Mag. A, 1984, vol. 49, no. 4, pp. 591-609. https://doi.org/10.1080/ $\underline{01418618408236558}$

[22] H.H. Kuo and T. Mura, Elastic field and strain energy of circular wedge disclination, J. Appl. Phys., 1972, vol. 43, no. 4, pp. 1454-1457. https://doi.org/10.1063/1.1661341

[23] J.D. Eshelby, A simple derivation of the elastic filed of an edge dislocation, Brit. J. Appl. Phys., 1966, vol. 17, no. 9, pp. 1131-1135. https://doi.org/10.1088/0508-3443/17/9/303

[24] M.A. Rozhkov, A.L. Kolesnikova, I.S. Yasnikov and A.E. Romanov, Disclination ensembles in graphene, Low Temp. Phys., 2018, vol. 44, no. 9, pp. 1171-1179. https://doi.org/10.1063/1.5052677

[25] W. Huang and T. Mura, Elastic fields and energies of a circular edge disclination and a straight screw disclination, J. Appl. Phys., 1970, vol. 41. no. 13, pp. 5175-5179. https://doi.org/10.1063/1.1658641

[26] A. Richter, A.E. Romanov, W. Pompe and V.I. Vladimirov, On the screening length of disclinations in amorphous structures, Phys. Stat. Sol. (b), 1987, vol. 143, no. 1, pp. 43-53. https://doi.org/10.1002/pssb.2221430105

[27] F. Kroupa and L. Lejcek, Elastic interaction between wedge disclinations, Phys. Stat. Sol. (b), 1972, vol. 51, no. 2, pp. K121-K124. https://doi.org/10.1002/pssb.2220510258

[28] V.G. Gryaznov, A.M. Kaprelov, I.A. Polonsky and A.E. Romanov, Disclinations in heterogeneous small particles, Phys. Stat. Sol. (b), 1991, vol. 167, no. 1, pp. 29-36. https://doi.org/10.1002/ pssb.2221670104

[29] M.S. Wu, A revisit of the elastic fields of straight disclinations with new solutions for a rigid core, Acta Mech., 2019, vol. 230, no. 7, pp. 2505-2520. https://doi.org/10.1007/s00707-019-02411-0

[30] Y.W. Liu, Q.H. Fang and C.P. Jiang, A wedge disclination dipole interacting with a circular inclusion, Phys. Stat. Sol. (a), 2006, vol. 203, no. 3, pp. 443-458. https://doi.org/10.1002/ pssa.200521049

[31] H.P. Song, Q.H. Fang and Y.W. Liu, The solution of a wedge disclination dipole interacting with an annular inclusion and the force acting on the disclination dipole, Chin. Phys. B, 2008, vol. 17, no. 2, pp. 4592-4598. https://doi.org/10.1088/ $\underline{1674-1056 / 17 / 12 / 043}$
[32] Y.X. Zhao, Q.H. Fang and Y.W. Liu, A wedge disclination dipole interaction with a coated cylindrical inhomogeneity, Acta Mech. Sol. Sinica, 2015, vol. 28, no. 1, pp. 62-73. https://doi.org/10.1016/S0894-9166(15)60016-7

[33] M.S. Wu, Elastic fields of a wedge disclination in functionally graded cylinder, Mech. Mater., 2021, vol. 157, art. 103835 . https://doi.org/10.1016/j.mechmat.2021.103835

[34] L. Lejcek, Magnetostrictive displacements at surface due to domain-wall junctions, Czech. J. Phys. B, 1978, vol. 28, no 4, pp. 434-441. https://doi.org/10.1007/BF01594255

[35] A.E. Romanov and V.I. Vladimirov, Straight wedge disclinations near a free surface, Phys. Stat. Sol. (a), 1980, vol. 59, no. 2, pp. K159-K163. https://doi.org/10.1002/pssa.2210590264

[36] A.E. Romanov and V.I. Vladimirov, Straight disclinations near a free surface. I. Stress fields, Phys. Stat. Sol. (a), 1981, vol. 63, no. 1, pp. 109118. https://doi.org/10.1002/pssa.2210630115

[37] A.E. Romanov, Straight disclinations near a free surface. II. The interaction between wedge disclination and surface, Phys. Stat. Sol. (a), 1981, vol. 63, no. 2, pp. 383-388. https://doi.org/10.1002/pssa.2210630202

[38] A.E. Romanov, Straight wedge disclinations in a two-phase material, Poverkhnost, 1985, vol. 12, pp. 36-42, in Russian.

[39] J. Dundurs and M. Hetenyi, The elastic plane with a circular insert, loaded by a radial force, J. Appl. Mech., 1961, vol. 83, no. 3, pp. 103-111. https://doi.org/10.1115/1.3640419

[40] V.I. Vladimirov and A.E. Romanov, The behavior of wedge disclination systems near grain boundaries, Metallofizika, 1982, vol. 4, no. 6, pp. 12-17, in Russian.

[41] V.I. Vladimirov, A.L. Kolesnikova and A.E. Romanov, Wedge disclinations in an elastic plate, Phys. Met. Metall., 1985, vol. 60, no. 6, pp. 58-67.

[42] A.L. Kolesnikova, N.D. Priemski and A.E. Romanov, Wedge straight disclinations in an elastic strip, Preprint no. 869, Ioffe PhysicalTechnical Institute, Leningrad, 1984, in Russian.

[43] A.E. Romanov, Disclination elastic fields in near surface layers, Poverkhnost, 1982, vol. 12, pp. 121-123, in Russian.

[44] A.L. Kolesnikova and A.E. Romanov, Edge dislocation perpendicular to the surfaces of a plate, Sov. Techn. Phys. Lett., 1987, vol. 13, no. 6, pp. 272-274.

[45] A.L. Kolesnikova and A.E. Romanov, Dislocation and disclination loops in the virtual-defect 
method, Phys. Sol. State, 2003, vol. 45, no. 9, pp. 1706-1718. https://doi.org/10.1134/1.1611238

[46] Ya.S. Uflyand, Integral Transformations in the Problems of Elasticity Theory, Nauka, Leninrad, 1975, in Russian.

[47] I.A. Polonsky, A.E. Romanov, V.G. Gryaznov and A.M. Kaprelov, Disclination in an elastic sphere, Phil. Mag. A, 1991, vol. 64, no. 2, pp. 281-287. https://doi.org/10.1080/ $\underline{01418619108221185}$

[48] A.L. Kolesnikova, M.Yu. Gutkin, A.V. Proskura, N.F. Morozov and A.E. Romanov, Elastic fields of straight wedge disclinations axially piercing bodies with spherical free surfaces, Int. J. Sol. Struct., 2016, vol. 99, pp. 82-96. https://doi.org/10.1016/j.ijsolstr.2016.06.029

[49] A.I. Lur'e, Three Dimensional Problems of the Theory of Elasticity, State Publishing House of Scientific and Technical Literature, Moscow, 1955, in Russian.

[50] A. Howie and L.D. Marks, Elastic strain and energy balance for multiply twinned particles, Phil. Mag. A, 1984, vol. 49, no. 1, pp. 95-109. https://doi.org/10.1080/01418618408233432

[51] V.G. Gryaznov, J. Heydenreich, A.M. Kaprelov, S.A. Nepijko, A.E. Romanov and J. Urban, Pentagonal symmetry and disclinations in small particles, Cryst. Res. Techn., 1999, vol. 134, pp. 1091-1119. https://doi.org/10.1002/(SICI)15214079(199911)34:9<1091::AIDCRAT1091>3.0. $\mathrm{CO} ; 2-\mathrm{S}$

[52] L.M. Dorogin, A.L. Kolesnikova and A.E. Romanov, Misfit layer formation in icosahedral nanoparticles, Techn. Phys. Lett., 2008, vol. 34, no. 9, pp. 779-781. https://doi.org/10.1134/ $\underline{\mathrm{S} 1063785008090198}$

[53] L.M. Dorogin, S. Vlassov, A.L. Kolesnikova, I. Kink, R. Lohmus and A.E. Romanov, Crystal mismatched layers in pentagonal nanorods and nanoparticles, Phys. Stat. Sol.(b), 2010, vol. 247, no. 2, pp. 288-298. https://doi.org/10.1002/ pssb.200945385

[54] V.I. Vladimirov and A.E. Romanov, Disclinations in Crystals, Nauka, Leningrad, 1986, in Russian.

[55] V.V. Rybin, Large Plastic Deformations and Ductile Fracture of Metals, Metallurgy, Moscow, 1986, in Russian.

[56] M.Yu. Gutkin and I.A. Ovid'ko, Plastic Deformation in Nanocrystalline Materials, Springer-Verlag Berlin Heidelberg, 2004.

[57] N.Yu. Zolotorevsky and V.V. Rybin, Fragmentation and Texture Formation During Deformation of Metallic Materials, Polytechnical University, St. Petersburg, 2014, in Russian.
[58] A.E. Romanov, Screened disclinations in solids, Mater. Sci. Eng. A, 1993, vol. 164, no. 1-2, pp. 5868. https://doi.org/10.1016/B978-1-4832-2815$\underline{0.50010-3}$

[59] A.A. Nazarov, Disclinations in bulk nanostructured materials: their origin, relaxation and role in material properties, Adv. Nat. Sci. Nanosci. Nanotechn., 2013, vol. 3, no. 4, art. 033002. https://doi.org/10.1088/2043-6262/4/3/ $\underline{033002}$

[60] A.E. Romanov, M.A. Rozhkov and A.L. Kolesnikova, Disclinations in polycrystalline graphene and pseudo-graphenes. Review, Lett. Mater., 2018, vol. 8, no. 4, pp. 384-400. https://doi.org/10.22226/2410-3535-2018-4-384-400

[61] N.D. Abramenko, M.A. Rozhkov, A.L. Kolesnikova and A.E. Romanov, Structure and Properties of Pseudo-Graphenes. Review, Rev. Adv. Mater. Tech., 2020, vol. 2, no. 4, pp.26. https://doi.org/10.17586/2687-0568-2020-2-4-9-26

[62] A.E. Romanov and A.L. Kolesnikova, Micromechanics of defects in functional materials, Acta Mech., 2021, vol. 232, no. 5, pp. 1901-1915. https://doi.org/10.1007/s00707-020$\underline{02872-8}$

[63] R.W. Armstrong, Wedge dislocation as the elastic counterpart of a crystal deformation twin, Science, 1968, vol. 68, no. 3855, pp. 799-800. https://doi.org/10.1126/science.162.3855.799

[64] A.H. King and Y.M. Zhu, Twin-corner disclinations in $\mathrm{YBa}_{2} \mathrm{Cu}_{3} \mathrm{O}_{7-\delta}$, Phil. Mag. A, 1993, vol. 67, no. 4, pp. 1037-1044. https://doi.org/ $\underline{10.1080 / 01418619308213974}$

[65] P. Müllner and A.E. Romanov, Between dislocation and disclination models for twins, Scripta Met. Mater., 1994, vol. 31, no. 12, pp. 1657-1662. https://doi.org/10.1016/0956716X(94)90459-6

[66] P. Müllner and A.E. Romanov, Internal twinning in deformation twinning, Acta Mater., 2000, vol. 48, no. 9, pp. 2323-2337. https://doi.org/10.1016/S1359-6454(00)00025-2

[67] P. Müllner and A. H. King, Deformation of hierarchically twinned martensite, Acta Mater., 2010, vol. 58, no. 16, pp. 5242-5261. http://dx.doi.org/10.1016/j.actamat.2010.05.048

[68] S.L. Thomas, A.H. King and D.J. Srolovitz, When twins collide: twin junctions in nanocrystalline nickel, Acta Mater., 2016, vol. 113, no. 2, pp. 301-310. http://dx.doi.org/10.1016/ j.actamat.2016.04.030

[69] P. Müllner, Twinning stress of type I and type II deformation twins, Acta Mater., 2019, vol. 176, 
no. 1, pp. 211-219. https://doi.org/10.1016/ j.actamat.2019.07.004

[70] N.Yu. Zolotorevsky and V.V. Rybin, Deformation of fragmenting polycrystals and texture formation, Fiz. Met. Metall., 1985, vol. 59, no. 3, pp. 440-449, in Russian.

[71] V.V. Rybin, N.Yu. Zolotorevsky and I.M. Zhukovskii, Structure evolution and internalstresses on stage of developed plasticdeformation of crystalline solids, Fiz. Met. Metall., 1990, vol. 59, no. 11, pp. 5-26, in Russian.

[72] V.V. Rybin, A.A. Zisman and N.Y. Zolotorevsky, Junction disclinations in plastically deformed crystals, Acta Met. Mater., 1993, vol. 47, no. 7, pp. 2211-221. https://doi.org/10.1016/09567151(93)90390-E

[73] P. Klimanek, V. Klemm, A.E. Romanov and M. Seefeldt, Disclinations in plastically deformed metallic materials, Adv. Eng. Mater., 2001, vol. 3, no. 11, pp. 877-884. https://doi.org/ 10.1002/1527-2648(200111)3:11<877::AIDADEM877>3.0.CO;2-L

[74] A.E. Romanov, Importance of disclinations in severe plastically deformed materials, Adv. Eng. Mater., 2003, vol. 5, no. 5, pp. 301-307. https://doi.org/10.1002/adem.200310087

[75] V.V. Rybin, V.N. Perevezentsev and Yu.V. Svirina, Model of formation of broken dislocation boundaries at joint disclinations, Techn. Phys., 2016, vol. 61, no. 6, pp. 898-903. https://doi.org/10.1134/S1063784216060190

[76] V.I. Vladimirov and A.E. Romanov, Partial disclination dipole motion under plastic deformation, Sov. Phys. Sol. State, 1978, vol. 20, no. 10, pp. 1795-1796.

[77] G.V. Berezhkova, P.P. Perstnev, A.E. Romanov and V.I. Vladimirov, Peculiarities of reoriented bands formation on crystals, Cryst. Res. Techn., 1983, vol. 18, no. 2, pp. 139-147. https://doi.org/10.1002/crat.2170180202

[78] B.K. Barakhtin, S.A. Ivanov, I.A. Ovid'ko, A.E. Romanov and V.I. Vladimirov, Periodic variations of defect structures in deformed crystals, J. Phys. D, 1989, vol. 22, no. 4, pp. 519-526. https://doi.org/10.1088/0022-3727/22/4/009

[79] M.Yu. Gutkin, K.N. Mikaelyan, A.E. Romanov and P. Klimanek, Disclination models for misorientation band generation and propagation, Phys. Stat. Sol. (a), 2002, vol. 193, no. 1, pp. 35-52. https://doi.org/10.1002/1521396X(200209)193:1<35::AID-PSSA35>3.0.CO;2-Y

[80] N.A. Pertsev, A.E. Romanov and V.I. Vladimirov, Disclination - dislocation model for the kink bands in polymers and fiber composites, $\mathrm{J}$. Mater. Sci., 1981, vol. 16, no. 8, pp. 2084-2090. https://doi.org/10.1007/BF00542368

[81] N.A. Pertsev and A.E. Romanov, Instability of front profiles of kink bands in oriented polymers, Mech. Comp. Mater., 1984, vol. 19, no. 5, pp. 565-570. https://doi.org/10.1007/bf00604454

[82] N.A. Pertsev, Plastic relaxation and disclination strain-hardening in composite-materials, Mech. Comp. Mater., 1987, vol. 23, no. 1, pp. ţ42-49. https://doi.org/10.1007/BF00815387

[83] T. Tokuzumi, S. Yamasaki, W. Li, M. Mitsuhara and H. Nakashima, Morphological and crystallographic features of kink bands in longperiod stacking ordered $M g-Z n-Y$ alloy analyzed by serial sectioning SEM-EBSD observation method, Materialia, 2020, vol. 12, art. 100716. https://doi.org/10.1016/j.mtla.2020.100716

[84] A.A. Nazarov, A.E. Romanov and R.Z. Valiev, On the nature of high internal stresses in ultra-fine grained materials, Nanostr. Mater., 1994, vol. 4, no. 1, pp. 93-101. https://doi.org/10.1016/09659773(94)90131-7

[85] A.A. Nazarov, A.E. Romanov and R.Z. Valiev, Models of the defects structure and analysis of the mechanical behavior of nanocrystals, Nanostr. Mater., 1995, vol. 5, no. 5-8, pp. 775-778. https://doi.org/10.1016/0965-9773(95)00174-3

[86] A.A. Nazarov, A.E. Romanov and R.Z. Valiev, Random disclination ensembles in ultrafinegrained materials produced by severe plastic deformation, Scripta Mater., 1996, vol. 34, no. 5, pp. 729-734. https://doi.org/10.1016/13596462(95)00573-0

[87] S.G. Zaichenko and A.M. Glezer, Disclination mechanism for plastic deformation of nanocrystalline materials, Phys. Sol. State, 1997, vol. 39, no. 11, pp. 1810-1914. https://doi.org/10.1023/A:1008714612121

[88] I.A. Ovid'ko, Materials science - Deformation of nanostructures, Science, 2002, np. 5564, pp. 2386-2386. https://doi.org/10.1126/ science.1071064

[89] M.Y. Gutkin, I.A. Ovid'ko and N.V. Skiba, Crossover from grain boundary sliding to rotational deformation in nanocrystalline materials, Acta Mater., 2003, vol. 51, no. 14, pp. 4059-4071. https://doi.org/10.1016/S13596454(03)00226-X

[90] I.A. Ovid'ko, R.Z. Valiev and Y.T. Zhu, Review on superior strength and enhanced ductility of metallic nanomaterials, Progr. Mater. Sci., 2018, 
vol. 94, pp. 462-540. https://doi.org/10.1016/ j.pmatsci.2018.02.002

[91] V.G. Gryaznov, M.Yu. Gutkin, A.E. Romanov and L.I. Trusov, On the yield stress of nanocrystals, J. Mater. Sci., 1993, vol. 28, no. 16, pp. 4359-4365. https://doi.org/10.1007/BF01154943

[92] A.L. Kolesnikova, I.A. Ovid'ko and A.E. Romanov, Dislocation-disclination transformations and the reverse Hall-Petch effect in nanocrystalline materials, Techn. Phys. Lett., 2007, vol. 33, no. 8, pp. 641-644. https://doi.org/10.1134/s1063785007080056

[93] A.E. Romanov, A.L. Kolesnikova, I.A. Ovid'ko and E.C. Aifantis, Disclinations in nanocrystalline materials: Manifestation of the relay mechanism of plastic deformation, Mater. Sci. Eng. A, 2009, vol. 503, no. 1-2, pp. 62-67. https://doi.org/10.1016/j.msea.2008.05.053

[94] I.A. Ovid'ko and A.G. Sheinerman, Grain boundary sliding, triple junction disclinations and strain hardening in ultrafine-grained and nanocrystalline metals, Int. J. Plast., 2017, vpl.96, pp. 227-241. https://doi.org/10.1016/ j.ijplas.2017.05.005

[95] P. Cordier, S. Demouchy and B. Beausir, Disclinations provide the missing mechanism for deforming olivine-rich rocks in the mantle, Nature, 2014, vol. 507, no. 7490, pp. 51-56. https://doi.org/10.1038/nature13043

[96] B.M. Moshtaghioun, J.A. Bejarano-Palma and D.G. García, Disclination dipoles are the Holy Grail for high temperature superplasticity in ceramics, Scripta Mater., 2020, vol. 185, pp. 21-24. https://doi.org/10.1016/ j.scriptamat.2020.03.049

[97] J.C.M. Li, Disclination model of high angle grain-boundaries, Surf. Sci., 1972, vol. 31, pp. 12-26. https://doi.org/10.1016/0039-6028(72) 90251-8

[98] K.K. Shih and J.C.M. Li, Energy of grainboundaries between cusp misorientations, Surf. Sci., 1975, vol. 50, no.1, pp. 109-124. https://doi.org/10.1016/0039-6028(75)90176-4

[99] V.Yu. Gertsman, A.A. Nazarov, A.E. Romanov, R.Z. Valiev and V.I. Vladimirov, Disclinationstructural unit model of grain boundaries, Phil. Mag. A, 1998, vol. 59, no. 5, pp. 1113-1118. https://doi.org/10.1080/01418618908209841

[100] A.A. Nazarov and A.E. Romanov, On the average misorientation of general tilt boundaries, Phil. Mag. Lett., 1998, vol. 60, no. 5, pp. 187-193. https://doi.org/10.1080/ $\underline{09500838908206456}$
[101] R.Z. Valiev, V.I. Vladimirov, V.Yu. Gertsman, A.A. Nazarov and A.E. Romanov, Disclinationstructural model and energy of grain boundaries in fcc metals, Phys. Met. Metall., 1990, vol. 69, no. 33, pp. 30-37.

[102] M.S. Wu, A.A. Nazarov and K. Zhou, Misorientation dependence of the energy of [1100] symmetrical tilt boundaries in hcp metals: prediction by the disclination-structural unit model, Phil. Mag., 2004, vol. 84, no. 8, pp. 785806. https://doi.org/10.1080/ 14786430310001646817

[103] O.A. Shenderova, D.W. Brenner, A.A. Nazarov, A.E. Romanov and L. Yang, Multiscale modeling approach for calculating grain boundaries energies from first principles, Phys. Rev. B, 1998, vol. 57, no. 6, pp. R3181-R3184. https://doi.org/10.1103/PhysRevB.57.R3181

[104] A.A. Nazarov, O.A. Shenderova and D.W. Brenner, Elastic models of symmetrical $<002>$ and $<011>$ tilt grain boundaries in diamond, Phys. Rev. B, 2000, vol. 61, no. 2, pp. 928-936. https://doi.org/10.1103/PhysRevB.61.928

[105] A.A. Nazarov, O.A. Shenderova and D.W. Brenner, On the disclination-structural unit model of grain boundaries, Mater. Sci. Eng. A, 2000, vol. 281, no. 1-2, pp. 148-155. https://doi.org/10.1016/S0921-5093(99)00727-3

[106] D.V. Bachurin, R.T. Murzaev and A.A. Nazarov, Atomistic computer and disclination simulation of [001] tilt boundaries in nickel and copper, Phys. Met. Metall., 2003, vol. 96, no. 6, pp. 555-561.

[107] A.A. Nazarov, A.E. Romanov and R.Z. Valiev, On the structure, stress fields and energy of nonequilibrium grain boundaries, Acta Met. Mater., 1993, vol. 41, no. 4, pp. 1033-1040. https://doi.org/10.1016/0956-7151(93)90152-I

[108] K. N. Mikaelyan, I.A. Ovid'ko and A.E. Romanov, Quasiperiodic tilt boundaries in polycrystalline and nanocrystalline materials: energy and stress fields, Mater. Sci. Eng. A, 1999, vol. 259, no. 1, pp. 132-137. https://doi.org/ $\underline{10.1016 / \text { S0921-5093(98)00874-0 }}$

[109] K.N. Mikaelyan, I.A. Ovid'ko and A.E. Romanov, Disclination-structural-unit model of grain boundaries of finite extent, Mater. Sci. Eng. A 288(1) (2000) 61-65. https://doi.org/10.1016/ S0921-5093(00)00884-4

[110] K.N. Mikaelyan, I.A. Ovid'ko and A.E. Romanov, Disclinations at quasiperiodic grain tilt boundaries, Phys. Met. Metall., 2000, vol. 90, no. 3, pp. 224-230. 
[111] A.A. Zisman and V.V. Rybin, Basic configurations of interfacial and junction defects induced in a polycrystal by deformation of grains, Acta Mat., 1996, vol. 44, no. 1, pp. 403407. https://doi.org/10.1016/1359-6454(95) $\underline{00155-8}$

[112] A.A. Zisman and V.V. Rybin, Mesoscopic stress field arising from the grain interaction in plastically deformed polycrystals, Acta Mat., 1998, vol. 46, no.2, pp. 457-464. : https://doi.org/ 10.1016/S1359-6454(97)00275-9

[113] V.Y. Gertsman, On the line defects associated with grain boundary junctions, Z. Metall., 2003, vol. 94, no. 10, pp. 1153-1156. https://doi.org/ $\underline{10.3139 / 146.031153}$

[114] T.S. Orlova, A.A. Nazarov, N.A. Enikeev, I.V. Alexandrov, R.Z. Valiev and A.E. Romanov, Grain size refinement due to relaxation of disclination junction configurations in the course of plastic deformation of polycrystals, Phys. Sol. State, 2005, vol. 47, no. 5, pp. 845-851. https://doi.org/10.1134/1.1924843

[115] A.A. Nazarov, N.A. Enikeev, T.S. Orlova, A.E. Romanov, I.V. Alexandrov and R.Z. Valiev, Disclination micromechanical simulation of grain subdivision in equal-channel angular pressing, Russian Metall., 2005, vol. 2005, no. 5, pp. 63-70.

[116] A.A. Nazarov, N.A. Enikeev, T.S. Orlova, A.E. Romanov, I.V. Alexandrov, I.J. Beyerlein and R.Z. Valiev, Analysis of substructure evolution during simple shear of polycrystals by means of combined viscoplastic self-consistent and disclination modeling approach, Acta Mater., 2006, vol. 54, no. 4, pp. 985-995. https://doi.org/ 10.1016/j.actamat.2005.10.025

[117] M. Seefeldt, A disclination-based approach for mesoscopic statistical modeling of grain subdivision in niobium, Comp. Mater. Sci., 2013, vol. 76, no. 1, pp. 12-19. https://doi.org/10.1016/ j.commatsci.2013.03.039

[118] A.A. Nazarov and D.V. Bachurin, On the relaxation of quadrupoles of junction disclinations in deformed polycrystals, Phys. Met. Metall., 2003, vol. 96, no. 5, pp. 446-451.

[119] D.V. Bachurin and A.A. Nazarov, On the annealing of junction disclinations in deformed polycrystals, Phil. Mag., 2003, vol. 83, no. 23, pp. 2653-2667. https://doi.org/10.1080/ 1478643031000137895

[120] A.L. Kolesnikova, V. Klemm, P. Klimanek and A.E. Romanov, Transmission electron microscopy image contrast of disclination defects in crystals (computer simulation), Phys. Stat. Sol. (a), 2002, vol. 191, no. 2, pp. 467-481. https://doi.org/10.1002/1521396X(200206)191:2<467::AIDPSSA467>3.0.CO;2-1

[121] P. Klimanek, V. Klemm, M. Motylenko and A.E. Romanov, Substructure analysis in heavily deformed materials by diffraction methods, Adv. Eng. Mater., 2004, vol. 6, no. 11, pp. 861-871. https://doi.org/10.1002/adem.200400125

[122] V.V. Rybin and I.M. Zhukovskii, Disclination mechanism of microcrack formation, Sov. Phys. Sol. State, 1978, vol. 20, no. 6, pp. 1829-1835.

[123] V.V. Rybin, A.A. Zisman and I.M. Zhukovskii, Microcrack formation under conditions of developed plastic strain, Strength Mater., 1982, vol. 14, pp. 1584-1590. https://doi.org/10.1007/ $\underline{\mathrm{BF} 00768642}$

[124] M.S. Wu and H. Zhou, Analysis of a crack in a disclinated cylinder, Int. J. Fract., 1996, vol. 82, no. 4,pp. 381-399. https://doi.org/10.1007/ $\underline{\mathrm{BF} 00013240}$

[125] M. Yu. Gutkin and I. A. Ovid'ko, Disclinations, amorphization and microcrack generation at grain boundary junctions in polycrystalline solids, Phil. Mag. A, 1994, vol. 70, no. 4, pp. 561-575. https://doi.org/10.1080/ $\underline{01418619408242248}$

[126] J. Luo, K. Zhou and Z.M. Xiao, Stress investigation on a Griffith crack initiated from an eccentric disclination in a cylinder, Acta Mech., 2009, vol. 202, no. 1, pp. 65-77. https://doi.org/10.1007/s00707-008-0010-1

[127] K. Zhou, A.A. Nazarov and M.S. Wu, Continuum and atomistic studies of a disclinated crack in a bicrystalline nanowire, Phys. Rev. B, 2006, vol. 73, no. 4, art. 045410. https://doi.org/10.1103/PhysRevB.73.045410

[128] M.S. Wu, K. Zhou and A.A. Nazarov, Crack nucleation at disclinated triple junctions, Phys. Rev. B, 2007, vol. 76, no. 13, art. 134105. https://doi.org/10.1103/PhysRevB.76.134105

[129] M.S. Wu, Characteristics of a disclinated Zener crack with cohesive end zones, Int. J. Eng. Sci., 2001, vol. 39, no. 13, pp. 1459-1485. https:// doi.org/10.4028/www.scientific.net/SSP.87.277

[130] M.S. Wu, Energy analysis of Zener-Griffith crack nucleation from a disclination dipole, Int. J. Plast., 2018, vol. 100, no. 1, pp. 142-155. https://doi.org/10.1016/j.ijplas.2017.10.001

[131] M.S. Wu, Crack nucleation from a wedge disclination dipole with shift of rotation axes, 
Int. J. Fract., 2018, vol. 212, no. 1, pp. 53-66. https://doi.org/10.1007/s10704-018-0292-9

[132] I.A. Ovid'ko and A.G. Sheinerman, Nanocrack generation at dislocation-disclination configurations in nanocrystalline metals and ceramics, Phys. Rev. B, 2008, vol. 77, no. 5, art. 054109. https://doi.org/10.1103/ PhysRevB.77.054109

[133] G.F. Sarafanov and V.N. Perevezentsev, $A$ criterion of nucleation of a microcrack in an elastic disclination field screened by an ensemble of dislocations, Techn. Phys. Lett., 2015, vol. 41, no. 10, pp. 968-970. https://doi.org/10.1134/S1063785015100144

[134] S.V. Kirikov and V.N. Perevezentsev, Analysis of the conditions for the existence of stable microcracks in an elastic stress field from a rotational-shear mesodefect, Lett. Mater., 2021, vol. 11, no. 1, pp. 50-54. https://doi.org/10.22226/ 2410-3535-2021-1-50-54

[135] I.A. Ovid'ko and A.G. Sheinerman, Generation of nanocracks at deformation twins in nanomaterials, Mater. Res. Lett., 2013, vol. 1, no. 3, pp. 168-173. https://doi.org/10.1080/ 21663831.2013.814091

[136] J. Lou, Study of microcrack nucleation from a blocked twin with the wedge disclination model, Arch. Appl. Mech., 2017, vol. 87, no. 1, pp. 75-85. https://doi.org/10.1007/s00419-016$\underline{1177-\mathrm{x}}$

[137] A.E. Romanov and G.G. Samsonidze, Diffusion in the elastic field of a wedge disclination, Sov. Techn. Phys. Lett., 1988, vol. 14, no. 4, pp. 585586.

[138] A.V. Osipov and I.A. Ovid'ko, Diffusion-induced decay of disclinations and solid-state amorphization in mechanically alloyed materials, Appl. Phys. A, 1992, vol. 54, no. 6, pp. 517-519. https://doi.org/10.1007/BF00324331

[139] V.M. Vlasov and V.A. Zaznoba, Diffusion processes near triple joints of special grain boundaries, Phys. Sol. State, 1999, vol. 41, no. 1, pp. 55-58. https://doi.org/10.1134/1.1130730

[140] R.T. Murzaev and A.A. Nazarov, Energies of formation and activation for migration of grain-boundary vacancies in a nickel bicrystal containing a disclination, Phys. Met. Metall., 2006, vol. 102, no. 2, pp. 198-204. https://doi.org/10.1134/S0031918X06080114

[141] V.M. Vlasov and I.I. Fedik, Structural and impurity traps for hydrogen atoms, Int. J. Hydr. Energy, 2006, vol. 31, no. 2, pp. 265-267. https://doi.org/10.1016/j.ijhydene.2005.04.059
[142] A.E. Romanov, I.A. Polonsky, V.G. Gryaznov, S.A. Nepijko, T. Junghaus and N.I. Vitrykhovski, Voids and channels in pentagonal crystals, J. Cryst. Growth, 1993, vol. 129, no. 3-4, pp. 691698. https://doi.org/10.1016/0022-0248(93) 90505-Q

[143] I.S. Yasnikov and A.A. Vikarchuk, The formation of voids in icosahedral small particles during electrocrystallization, Techn. Phys. Lett., 2007, vol. 33, no. 10, pp. 817-820. https://doi.org/10.1134/S1063785007100045

[144] R. de Wit, Partial disclinations, J. Phys. C, 1972, vol. 5, no. 5, pp. 529-534. https://doi.org/10.1088/0022-3719/5/5/004

[145] J.M. Galligan, Fivefold symmetry and disclinations, Scripta Met., 1972, vol. 6, no. 1, pp. 161-144. https://doi.org/10.1016/00369748(72)90269-4

[146] L.I. Trusov, M.Yu. Tanakov, V.G. Gryaznov, A.M. Kaprelov and A.E. Romanov, Relaxation of elastic stresses in overlayed microcrystals, J. Cryst. Growth, 1991, vol. 114, no. 2, pp. 133-140. https://doi.org/10.1016/00220248(91)90688-2

[147] V.G. Gryaznov, A.M. Kaprelov, A.E. Romanov and I.A. Polonsky, Channels of relaxation of elastic stresses in pentagonal nanoparticles, Phys. Stat. Sol. (b), 1991, vol. 176, no. 2, pp. 441450. https://doi.org/10.1002/pssb.2221670206

[148] A.E. Romanov, A.A. Vikarchuk, A.L. Kolesnikova, L.M. Dorogin, I. Kink and E.C. Aifantis, Structural transformations in nanoand microobjects triggered by disclinations, J. Mater. Res., 2012, vol. 27, no. 3, pp. 545-551. https://doi.org/10.1557/jmr.2011.372

[149] A.L. Kolesnikova and A.E. Romanov, Stress relaxation in pentagonal whiskers, Techn. Phys. Lett., 2007, vol. 33, no. 10, pp. 886-888. https://doi.org/10.1134/S1063785007100239

[150] M.Y. Gutkin, A.L. Kolesnikova, S.A. Krasnitckii, L.M. Dorogin, V.S. Serebryakova, A.A. Vikarchuk and A.E. Romanov, Stress relaxation in icosahedral small particles via generation of circular prismatic dislocation loops, Scripta Mater., 2015, vol. 105, no.1, pp. 10-13. https://doi.org/10.1016/j.scriptamat.2015.04.01

[151] M.Yu. Krauchanka, S.A. Krasnitckii, M.Yu. Gutkin, A.L. Kolesnikova, A.E. Romanov and E.C. Aifantis, Generation of circular prismatic dislocation loops in decahedral small particles, Scripta Mater., 2018, vol. 146, no. 1, pp. 77-81.

[152] I.S. Yasnikov, A.L. Kolesnikova and A.E. Romanov, Multi-disclination description of 
pentagonal particles with subsurface layer free of twin boundaries, Phil. Mag. Lett., 2015. vol. 95, no. 9, pp. 450-457. https://doi.org/10.1080/ 09500839.2015.1085130

[153] I.S. Yasnikov, A.L. Kolesnikova and A.E. Romanov, Multi-disclination configurations in pentagonal microcrystals and two-dimensional carbon structures, Phys. Sol. State, 2016, vol. 58, no. 6, pp. 1184-1190. https://doi.org/10.1134/ $\underline{\mathrm{S} 1063783416060342}$

[154] M.Yu. Gutkin, A.L. Kolesnikova, I.S. Yasnikov, A.A. Vikarchuk, E.C. Aifantis and A.E. Romanov, Stresses and fracture in hollow decahedral small particles, Eur. J. Mech. A, 2018, vol. 68, no. 1, pp. 133-139. https://doi.org/10.1016/ j.euromechsol.2017.11.004

[155] A.L. Kolesnikova and A.E. Romanov, Formation of mismatched layers in pentagonal nanorods, Phys. Stat. Sol. RRL, 2007, vol. 1, no. 6, pp. 271273. https://doi.org/10.1002/PSSR.200701204

[156] L.M. Dorogin, S. Vlassov, A.L. Kolesnikova, I. Kink, R. Lõhmus and A.E. Romanov, Pentagonal nanorods and nanoparticles with mismatched shell layers, J. Nanosci. Nanotechn., 2010, vol. 10, no. 9, pp. 6136-6143. https://doi.org/10.1166/jnn.2010.2563

[157] N. Rivier, Disclination lines in glasses, Phil. Mag. A, 1979, vol. 40, no. 6, pp. 859-868. https://doi.org/10.1080/01418617908234879

[158] M. Kleman, Dual properties of conjugate disclination segment networks in amorphous materials, J. de Phys. Lett., 1983, vol. 44, no. 8, pp. L295-L302. https://doi.org/10.1051/ jphyslet:01983004408029500

[159] A. Richter, A.E. Romanov, W. Pompe and V.I. Vladimirov, Geometry and energy of disclinations in topologically disordered systems, Phys. Stat. Sol. (b), vol. 122, no. 1, pp. 35-45. https://doi.org/10.1002/pssb.2221220104

[160] J.-F. Sadoc and R. Mosseri, Modeling of the structure of glasses, J. Non-Crystall. Sol., vol. 61-62, no. 1, pp. 487-498. https://doi.org/10.1016/ 0022-3093(84)90595-7

[161] V.A. Likhachev, A.I. Milhailin and L.V. Zhigilei, Molecular-dynamics study of medium-range order in metallic glasses, Phil. Mag. A, 1994, vol. 69 , no. 3, pp. 421-436. https://doi.org/10.1080/01418619408242222

[162] R. Mosseri and J.-F. Sadoc, Frustration and defects in non-periodic solids, Comp. Rend. Phys., 2014, vol. 15, no. 1, pp. 90-99. https://doi.org/10.1016/J.CRHY.2013.09.006

[163] M.Yu. Gutkin, I.A. Ovid'ko and A.E. Romanov, Intersection of dislocations with disclinations and flow stress in metallic glasses, Rad. Eff. Def. Sol., 1994, vol. 129, no. 2-4, pp. 239-255. https://doi.org/10.1080/10420159408229023

[164] S.V. Bobylev, I.A. Ovid'ko, A.E. Romanov and A.G. Sheinerman, Nanoscale defect structures at crystal-glass interfaces, J. Phys. Cond. Matter., 2005, vol. 17, no. 4, pp. 619-634. https://doi.org/10.1088/0953-8984/17/4/005

[165] A.K. Tagantsev, L.E. Cross and J. Fousek, Domains in Ferroic Crystals and Thin Films, Springer-Verlag, New York, 2010.

[166] J.S. Speck, A. Seifert, W. Pompe and R. Ramesh, Domain configurations due to multiple misfit relaxation mechanisms in epitaxial ferroelectric thin films. II. Experimental verification and implications, J. Appl. Phys., 1994, vol. 76, no. 1, pp. 477-483. https://doi.org/10.1063/1.357098

[167] O.I. Lebedevy, G. VanTendelooz, S. Amelinckx, F. Razavi and H.-U. Habermeier, Periodic microtwinning as a possible mechanism for the accommodation of the epitaxial film-substrate mismatch in the $\mathrm{La}_{1-x} \mathrm{Sr}_{x} \mathrm{MnO}_{3} \mathrm{SrTiO}_{3}$ system, Phil. Mag. A, 2001, vol. 81, no. 4, pp. 797-824. https://doi.org/10.1080/01418610151133230

[168] J.S. Speck, A.C. Daykin, A. Seifert, A.E. Romanov and W. Pompe, Domain configurations due to multiple misfit relaxation mechanisms in epitaxial ferroelectric thin films. III. Interfacial defects and domain misorientations, J. Appl. Phys., 1995, vol. 78, no. 3, pp. 1696-1706. https://doi.org/10.1063/1.360267

[169] N.A. Pertsev and A.G. Zembilgotov, Energetics and geometry of 90-degrees domain-structures in epitaxial ferroelectric and ferroelastic films, J. Appl. Phys., 1995, vol. 78, no. 10, pp. 61706180. https://doi.org/10.1063/1.360561

[170] A.E. Romanov, W. Pompe and J.S. Speck, Theory of microstructure and mechanics of the ...all a2/a1/a2... domain pattern in epitaxial ferroelectric and ferroelastic films, J. Appl. Phys., 1996, vol. 79, no. 8, pp. 4037-4049. https://doi.org/10.1063/1.361866

[171] N.A. Pertsev and A.G. Zembilgotov, Domain populations in epitaxial ferroelectric thin films: Theoretical calculations and comparison with experiment, J. Appl. Phys., 1996, vol. 80, no. 11,pp. 6401-6406. https://doi.org/10.1063/ $\underline{1.363659}$

[172] S.K. Streiffer, C.B. Parker, A.E. Romanov, M.J. Lefevre, L. Zhao, J.S. Speck, W. Pompe, C.M. Foster and G.R. Bai, Domain patterns in epitaxial rhombohedral ferroelectric films. I. Geometry and experiments, J. Appl. 
Phys.,1998, vol.83, no. 5, pp. 2742-2753.

https://doi.org/10.1063/1.366632

[173] A.E. Romanov, M.J. Lefevre, J.S. Speck, W. Pompe, S.K. Streiffer and C.M. Foster, Domain patterns in epitaxial rhombohedral ferroelectric films. II. Interfacial defects and energetics, J. Appl. Phys., 1998, vol. 83, no. 5, pp. 2754-2765. https://doi.org/10.1063/1.366636

[174] A.E. Romanov, A. Vojta, W. Pompe, M.J. Levere and J.S. Speck, Domain patterns in (111) oriented tetragonal ferroelectric films, Phys. Stat. Sol. (a), 1999, vol. 172, no. 1, pp. 225-253. https://doi.org/10.1002/(SICI)1521396X(199903)172:1<225::AIDPSSA225>3.0.CO;2-2

[175] A. Ullrich, W. Pompe, J.S. Speck and A.E. Romanov, Peculiarities of domain patterns in epitaxially grown ferroelectric thin films, Sol. State Phenom., 20025, vol. 87, pp. 245-254. https://doi.org/10.4028/www.scientific.net/ $\underline{\text { SSP.87.245 }}$

[176] N. Farag, M. Bobeth, W. Pompe and A.E. Romanov, J.S. Speck, Modeling of twinning in epitaxial (001)-oriented $\mathrm{La}_{0.67} \mathrm{Sr}_{0.33} \mathrm{MnO}_{3}$ thin films, J. Appl. Phys., 2005, vol. 97, no. 11, art. 113516. https://doi.org/10.1063/1.1914950

[177] N. Farag, M. Bobeth, W. Pompe and A.E. Romanov, Modelling of structural domains and elastic strain calculation in rhombohedral $\mathrm{La}_{1-x} \mathrm{Sr}_{x} \mathrm{MnO}_{3}$ films on (110) $\mathrm{SrTiO}_{3}$, Phil. Mag., 2007, vol. 87, no. 6, pp. 823-842. https://doi.org/10.1080/14786430600993331

[178] A.L. Kolesnikova and A.E. Romanov, $A$ disclination based approach to describing the structure of fullerenes, Phys. Sol. State, 1998, vol. 40, no. 6, pp. 1075-1077. https://doi.org/10.1134/1.1130490

[179] L.Y. Zhu, J.L. Ding and F. Ding, The great reduction of a carbon nanotube's mechanical performance by a few topological defects, ACS Nano, 2016, vol. 10, no. 6, pp. 6410-6415. https://doi.org/10.1021/acsnano.6b03231

[180] M. Ge and K. Sattler, Observation offullerene cones, Chem. Phys. Lett., 1994, vol. 220, no. 3-4, pp. 192-196. https://doi.org/10.1016/00092614(94)00167-7

[181] A.E. Romanov and A.G. Sheinerman, Energy of deformed and defective carbon clusters, Phys. Sol. State, 2000, vol. 42, no. 8, pp. 1569-1574. https://doi.org/10.1134/1.1307072

[182] A.E. Romanov, A.L. Kolesnikova, T.S. Orlova, I. Hussainova, V.E. Bougrov and R.Z. Valiev, Non-equilibrium grain boundaries with excess energy in graphene, Carbon, 2015, vol. 81, no. 1, pp. 223-231. https://doi.org/10.1016/ J.CARBON.2014.09.053

[183] A.L. Kolesnikova, M.A. Rozhkov, N.D. Abramenko and A.E. Romanov, On mesoscopic description of interfaces in graphene, Phys. Compl. Syst., 2020, vol. 1, no. 4, pp.; 129-134. https://doi.org/10.33910/2687-153X-2020-1-4129-134

[184] R. Majidi, Helium adsorption on carbon nanocones with different disclination angle: molecular dynamics simulation, Nano, 2012, vol. 7, no. 3, art. 1250023 . https://doi.org/10.1142/S1793292012500233

[185] M.A. Rozhkov, A.L. Kolesnikova, T.S. Orlova, L.V. Zhigilei and A.E. Romanov, Disclinated rings as structural units in MD simulation of intercrystallite boundaries in graphene, Mater. Phys. Mech., 2016, vol. 29, no. 1, pp. 101-105. https://www.ipme.ru/e-journals/MPM/no_12916/ MPM129_11_rozhkov.pdf

[186] A.L. Kolesnikova, M.A. Rozhkov, I. Hussainova, T.S. Orlova, I.S. Yasnikov, L.V. Zhigilei and A.E. Romanov, Structure and energy of intercrystallite boundaries in graphene, Rev. Adv. Mater. Sci., 2017, vol. 52, no. 1/2, pp. 91-98. https://www.ipme.ru/e-journals/RAMS/ no $15217 / 12 \_15217$ kolesnikova.pdf

[187] M.A. Rozhkov, N.D. Abramenko, A.L. Kolesnikova and A.E. Romanov, Zero misorientation interfaces in graphene, Lett. Mater., 2020, vol. 10, no. 4s, pp. 551-557. https://doi.org/10.22226/2410-3535-2020-4-551$\underline{557}$

[188] I.A. Ovid'ko and A.G. Sheinerman, Cracks at disclinated grain boundaries in graphene, J. Phys. D, 2013, vol. 46, no. 34, art. 345305. https://doi.org/10.1088/0022-3727/46/34/345305

[189] Z.H. Wang, X.F. Zhou, X.M. Zhang, Q. Zhu, H.F. Dong, M.M. Zhao and A.R. Oganov, Phagraphene: A low-energy graphene allotrope composed of 5-6-7 carbon rings with distorted Dirac cones, Nano Lett., 2015, vol. 15, no. 9, pp. 6182-6186. https://doi.org/10.1021/ acs.nanolett.5b02512

[190] Q. Fan, L. Yan, M. W. Tripp, O. Krejčí, S. Dimosthenous, S.R. Kachel, M. Chen, A.S. Foster, U. Koert, P. Liljeroth and J.M. Gottfried, Biphenylene network: A nonbenzenoid carbon allotrope, Science, 2021, vol. 372, no. 6544, pp. 852-856. https://doi.org/10.1126/science.abg4509

[191] T.-W. Chou and Y.C. Pan, Elastic energies of disclinations in hexagonal crystals, J. Appl. 
Physics, 1973, vol. 44, no. 1, pp. 63-65. https://doi.org/10.1063/1.1661940

[192] N.A. Pertsev, Disclinations in transversely isotropic media. 2. Angular and straight disclinations, Czech. J. Phys., 1983, vol. 2, pp. 199-207. https://doi.org/10.1007/BF01605499

[193] U. Zastrow, On the complete system of fundamental solutions for anisotropic slices and slabs: A comparison by use of the slab analogy, J. Elast., 1985, vol. 15, no. 3, pp. 293-318. https://doi.org/10.1007/bf00041427

[194] U. Zastrow, Basic geometrical singularities in plane elasticity and plate-bending problems, Int. J. Sol. Struct., 1985, vol. 21, no. 10, pp. 1047-1067. https://doi.org/10.1016/00207683(85)90055-1

[195] M.S. Wu, Stress and strain energy of a periodic array of interfacial wedge disclination dipoles in a transversely isotropic bicrystal, Int. J. Eng. Sci., 2002, vol. 40, no. 8, pp. 873-897. https://doi.org/10.1016/S0020-7225(01)00090-8

[196] K. Zhou and M.S. Wu, Exact solutions for periodic interfacial wedge disclination dipoles in a hexagonal bicrystal, Math. Mech. Sol., 2006, vol. 11, no. 4, pp. 337-360. https://doi.org/10.1177/1081286504040400

[197] M.S. Wu, K. Zhou and A.A. Nazarov, Stability and relaxation mechanisms of a wedge disclination in an HCP bicrystalline nanowire, Mod. Simul. Mater. Sci. Eng., 2006, vol. 14, no. 4, pp. 647-661. https://doi.org/10.1088/0965-0393/ $14 / 4 / 008$

[198] V.I. Vladimirov, I.A. Polonskii and A.E. Romanov, Nonlinear effects in elastic field of disclinations, Sov. Phys. Techn. Phys., 1988, vol. 58, no. 8, pp. 882-885.

[199] A. Seeger and A.E. Romanov, Die Wechselwirkung zwischen Schraubenverzetzung and Keildisklination, In: Verhandlungen der Deutschen Physikalischen Geselschaft "150 Jahre Deutsche Physikalische Gesellschaft. 59. Physikertagung Berlin”, Berlin, 1995, pp. 1469-1469.

[200] Yu.Z. Povstenko, Straight disclinations in nonlocal elasticity, Int. J. Eng. Sci., 1995, vol. 33, no. 4, pp. 575-582. https://doi.org/10.1016/ 0020-7225(94)00070-0

[201] L.M. Zubov, Nonlinear Theory of Dislocations and Disclinations in Elastic Bodies, Springer, Berlin, 1997.

[202] A. Yavari, On the wedge dispiration in an inhomogeneous isotropic nonlinear elastic solid, Mech. Res. Comm., 2016, vol. 78(B), pp.

55-59. https://doi.org/10.1016/

j.mechrescom.2016.02.008

[203] M.S. Wu, A wedge disclination in a nonlinear elastic cylinder, Math. Mech. Sol., 2019, vol. 24, no. 7, pp. 2030-2046. https://doi.org/10.1177/ 1081286518811399

[203] M.Yu. Gutkin and E.C. Aifantis, Dislocations and disclinations in the gradient theory of elasticity, Phys. Sol. State, 1999, vol. 41, no. 12, pp. 1980-1988. https://doi.org/10.1134/1.1131139

[204] M. Lazar and G.A. Maugin, Nonsingular stress and strain fields of dislocations and disclinations in first strain gradient elasticity, Int. J. Eng. Sci., 2005, vol. 43, no. 13-14, pp. 11571184. https://doi.org/10.1016/ j.ijengsci.2005.01.006

[205] J. Luo and F. Liu, Stress analysis of a wedge disclination dipole interacting with a circular nanoinhomogeneity, Eur. J. Mech. A, 2011, vol. 30, pp. 22-32. https://doi.org/10.1016/ j.euromechsol.2010.09.005

[206] S. Rezazadeh Kalehbasti, M.Yu. Gutkin and H.M. Shodja, Wedge disclinations in the shell of a core-shell nanowire within the surfacel interface elasticity, Mech. Mater., 2014, vol. 68, pp. 45-63. https://doi.org/10.1016/ j.mechmat.2013.08.003

[207] J.D. Clayton, D.L. McDowell and D.J. Bammann, Modeling dislocations and disclinations with finite micropolar elastoplasticity, Int. J. Plast., 2006, vol. 22, no. 2, pp. 210-256. https://doi.org/10.1016/j.ijplas.2004.12.001

[208] M.I. Karyakin and L.M. Zubov, Theory of isolated and continuously distributed disclinations and dislocations in micropolar media, In: Mechanics of Generalized Continua, ed. by H. Altenbach, G.A. Maugin and N. Verichev, vol. 7, Springer-Verlag, Berlin Heidelberg, 2011, pp. 275-290.

[208] A. Kadich and L. Edelen, Gauge Theory of Dislocations and Disclinations, Mir, Moscow, 1987, in Russian.

[209] I.A. Ovid'ko and A.E. Romanov, Topological excitations (defects, solitons, textures, frustrations) in condensed media, Phys. Stat. Sol. (a), 1987, vol. 104, no. 1, pp. 13-45. https://doi.org/10.1002/pssa.2211040102

[210] G. Gremaud, Universe and Matter conjectured as 3-dimensional Lattice with Topogical Singularities, Gérard Gremaud, Lausanne, 2016. 